\title{
Aptima $^{\circledR}$ Chlamydia trachomatis Assay
}

For in vitro diagnostic use.

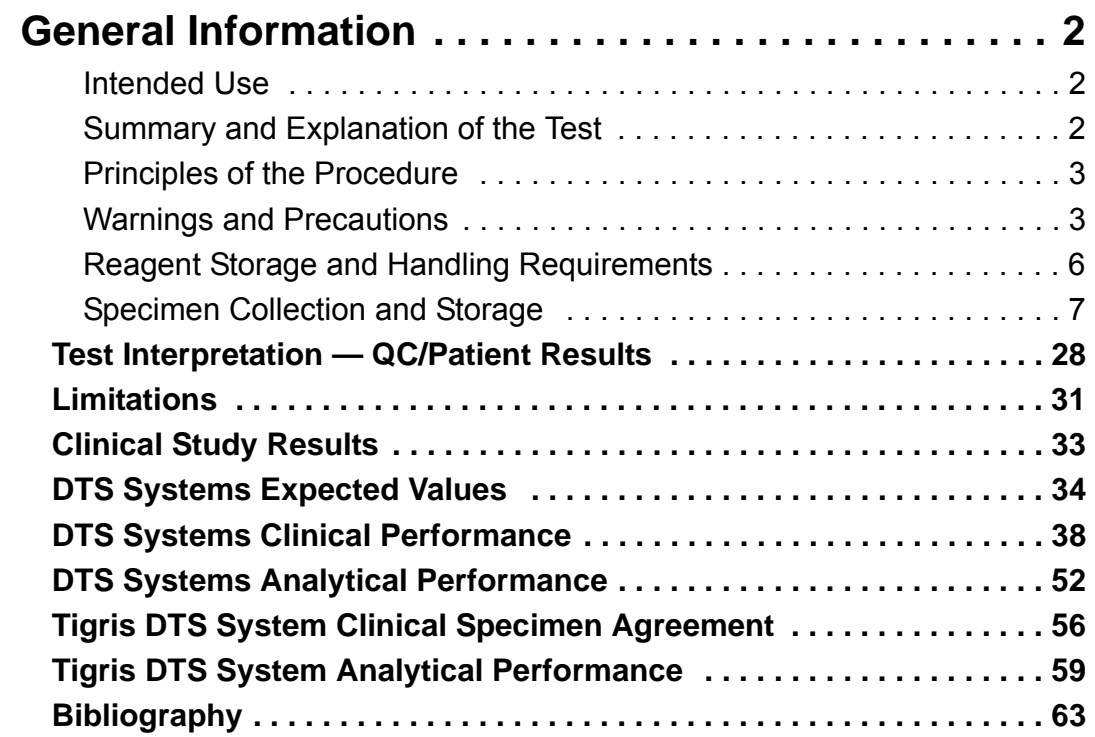

\section{DTS ${ }^{\circledR}$ Systems}

DTS Systems

Reagents and Materials Provided

.9

Materials Required But Available Separately

Optional Materials

10

DTS Systems Test Procedure

Procedural Notes

\section{Tigris $^{\circledast}$ DTS $^{\circledast}$}

Tigris DTS System

Reagents and Materials Provided ..............................21

Materials Required But Available Separately ...............23

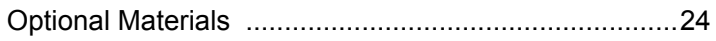

Tigris DTS System Test Procedure ................................24

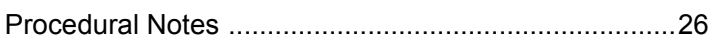




\section{General Information}

\section{Intended Use}

The Aptima ${ }^{\circledR}$ Chlamydia trachomatis Assay is a target amplification nucleic acid probe test that utilizes target capture for the in vitro qualitative detection of ribosomal RNA (rRNA) from Chlamydia trachomatis (CT) to aid in the diagnosis of chlamydial urogenital disease using the Tigris $^{\circledR}$ DTS $^{\circledast}$ Automated Analyzer or semi-automated instrumentation as specified. The assay may be used to test the following specimens from symptomatic individuals: cliniciancollected endocervical, vaginal and male urethral swab specimens; and female and male urine specimens. The assay may be used to test the following specimens from asymptomatic individuals: clinician-collected endocervical, vaginal and male urethral swab specimens; patient-collected vaginal swab specimens ${ }^{1}$; and female and male urine specimens. This assay is also intended for use with the testing of gynecological specimens, from both symptomatic and asymptomatic patients, collected in the PreservCyt ${ }^{\circledR}$ Solution.

${ }^{1}$ Patient-collected vaginal swab specimens are an option for screening women when a pelvic exam is not otherwise indicated. The vaginal swab specimen collection kit is not for home use.

\section{Summary and Explanation of the Test}

Chlamydia trachomatis infections are one of the most common sexually transmitted infections worldwide. In the United States alone, an estimated 1,307,893 (426.0 cases per 100,000 population) new cases of CT infections were reported to the Centers for Disease Control in $2010(5)$.

Chlamydiae are nonmotile, gram-negative, obligate intracellular bacteria. The CT species is comprised of fifteen serovars (A, B, Ba, C, D, E, F, G, H, I, J, K, L1, L2 and L3) that can cause disease in humans (27). The serovars $D$ through $K$ are the major cause of genital chlamydial infections in men and women (19). C. trachomatis can cause nongonococcal urethritis, epididymitis, proctitis, cervicitis, acute salpingitis, and pelvic inflammatory disease $(3,13,21,22)$. C. trachomatis infections are often asymptomatic in both males and females. Children born to infected mothers are at significantly higher risk for inclusion conjunctivitis and chlamydial pneumonia $(1,10,20)$.

Historically, several methods for CT detection have been utilized in the clinical laboratory, including cell culture, direct fluorescent antibody testing, and enzyme immunoassay. More recent methodologies for CT detection include direct DNA probe assays and nucleic acid amplification tests (NAATs). Cell culture was once considered to be the "gold standard" for detection of CT. Culture is quite specific, but recent publications have demonstrated that NAATs have a higher clinical sensitivity than culture $(2,8,14,23)$. Due to its lower clinical sensitivity and variable performance between laboratories, culture has been replaced in many laboratories by direct DNA probe and NAATs.

First generation NAATs for CT have technological issues that have limited their performance. These issues include cumbersome specimen processing and specimen inhibition that can yield false negative results $(6,12,15,18,24,26)$. The Aptima Chlamydia trachomatis Assay (Aptima CT Assay) is a second generation NAAT that utilizes target capture, TranscriptionMediated Amplification $\left(\mathrm{TMA}^{\circledR}\right.$ ), and Hybridization Protection Assay (HPA) technologies to streamline specimen processing, amplify target rRNA, and detect amplicon, respectively. Studies comparing performance and specimen inhibition of various amplification systems have demonstrated the benefits of target capture, TMA, and HPA $(7,11)$. 
According to Chlamydia trachomatis and Neisseria gonorrhoeae 2002 Screening Guidelines, CDC recommends a number of options for follow-up on a positive screening test "if a low positive predictive value can be expected or if a false-positive result would have serious psychosocial or legal consequences" (4). One of these options for additional testing can be a different FDA-cleared nucleic acid amplification test that targets a different nucleic acid sequence than the initial test. The Aptima CT Assay targets different nucleic acid sequences than those targeted by other $C$. trachomatis NAATs, including the Aptima Combo $2^{\circledR}$ Assay.

\section{Principles of the Procedure}

The Aptima CT Assay combines the technologies of target capture, TMA, and HPA.

Specimens are collected and transferred into their respective specimen transport tubes. The transport solution in these tubes releases the rRNA target and protects it from degradation during storage. When the Aptima CT Assay is performed in the laboratory, the target rRNA molecule is isolated from the specimens by use of a capture oligomer via target capture that utilizes magnetic microparticles. The capture oligomer contains a sequence complementary to a specific region of the target molecule as well as a string of deoxyadenosine residues. During the hybridization step, the sequence specific region of the capture oligomer binds to a specific region of the target molecule. The capture oligomer:target complex is then captured out of solution by decreasing the temperature of the reaction to room temperature. This temperature reduction allows hybridization to occur between the deoxyadenosine region on the capture oligomer and the poly-deoxythymidine molecules that are covalently attached to the magnetic particles. The micro particles, including the captured target molecule bound to them, are pulled to the side of the reaction vessel using magnets and the supernatant is aspirated. The particles are washed to remove residual specimen matrix that may contain amplification reaction inhibitors. After the target capture steps are completed, the specimens are ready for amplification.

Target amplification assays are based on the ability of complementary oligonucleotide primers to specifically anneal and allow enzymatic amplification of the target nucleic acid strands. The Hologic ${ }^{\circledR}$ TMA reaction replicates a specific region of the $16 \mathrm{~S}$ rRNA from CT via DNA intermediates. A unique set of primers is used for the target molecule. Detection of the rRNA amplification product sequences (amplicon) is achieved using nucleic acid hybridization. A single-stranded chemiluminescent DNA probe, which is complementary to a region of the target amplicon, is labeled with an acridinium ester molecule. The labeled DNA probe combines with amplicon to form stable RNA:DNA hybrids. The Selection Reagent differentiates hybridized from unhybridized probe, eliminating the generation of signal from unhybridized probe. During the detection step, light emitted from the labeled RNA:DNA hybrids is measured as photon signals in a luminometer, and are reported as Relative Light Units (RLU).

\section{Warnings and Precautions}

A. For in vitro diagnostic use.

B. For additional specific warnings, precautions and procedures to control contamination for the Tigris DTS ${ }^{\circledR}$ System, consult the Tigris DTS System Operator's Manual.

\section{Laboratory Related}

C. Use only supplied or specified disposable laboratory ware. 
D. Use routine laboratory precautions. Do not eat, drink or smoke in designated work areas. Wear disposable, powderless gloves, protective eye wear, and laboratory coats when handling specimens and kit reagents. Wash hands thoroughly after handling specimens and kit reagents.

E. Warning: Irritants, Corrosives. Avoid contact of Auto Detect 1 and Auto Detect 2 with skin, eyes and mucous membranes. If these fluids come into contact with skin or eyes, wash with water. If these fluids spill, dilute the spill with water before wiping dry.

F. Work surfaces, pipettes, and other equipment must be regularly decontaminated with $2.5 \%$ to $3.5 \%(0.35 \mathrm{M}$ to $0.5 \mathrm{M})$ sodium hypochlorite solution.

\section{DTS Systems Specific}

G. A separate area for HPA is strongly recommended to minimize amplicon contamination in the assay. This dedicated area should be away from the reagent preparation, target capture, and amplification areas.

$\mathrm{H}$. To help prevent lab areas from becoming contaminated with amplicon, the laboratory area should be arranged with a unidirectional workflow: from reagent preparation through HPA. Specimens, equipment, and reagents should not be returned to the area where a previous step was performed. Also, personnel should not move back into previous work areas without observing proper contamination safeguards.

\section{Specimen Related}

I. For the collection of endocervical and male urethral swab specimens, use only the Aptima Unisex Swab Specimen Collection Kit for Endocervical and Male Urethral Swab Specimens. For urine specimen collection, use only the Aptima Urine Specimen Collection Kit for Male and Female Urine Specimens. For clinician- and patient-collected vaginal swab specimens, use only the Aptima Vaginal Swab Specimen Collection Kit.

J. Expiration dates listed on the collection kits pertain to the collection site and not the testing facility. Samples collected any time prior to the expiration date of the collection kit, and transported and stored in accordance with the package insert, are valid for testing even if the expiration date on the collection tube has passed.

K. The PreservCyt Solution has been validated as an alternative medium for testing with the Aptima CT Assay. PreservCyt Solution liquid Pap specimens processed using the ThinPrep $^{\circledast} 3000$ Processor or other instruments have not been evaluated to test for Chlamydia trachomatis using the Aptima CT Assay.

L. After urine has been added in the urine transport tube, the liquid level must fall between the two black indicator lines on the tube label. Otherwise, the specimen must be rejected.

M. Maintain proper storage conditions during specimen shipping to ensure the integrity of the specimen. Specimen stability under shipping conditions other than those recommended has not been evaluated.

N. Specimens may be infectious. Use Universal Precautions when performing this assay. Proper handling and disposal methods should be established by the laboratory director. Only personnel adequately trained in handling infectious materials should be permitted to perform this diagnostic procedure. 
O. Avoid cross-contamination during the specimen handling steps. Specimens can contain extremely high levels of organisms. Ensure that specimen containers do not contact one another, and discard used materials without passing them over open containers. Change gloves if they come in contact with specimen.

P. If the lab receives a swab specimen transport tube with no swab, two swabs, a cleaning swab, or a swab not supplied by Hologic, the specimen must be rejected. Prior to rejecting a swab transport tube with no swab, verify that it is not an Aptima Specimen Transfer Tube as this specimen transport tube will not contain a swab.

Q. For PreservCyt Solution liquid Pap specimens, collect according to the manufacturer's instructions. Aliquots subsequently removed from the PreservCyt vial for testing by the Aptima CT Assay should be processed using only the Aptima Specimen Transfer Kit.

R. Upon piercing, liquid can discharge from Aptima transport tube caps under certain conditions. Follow instructions in the appropriate Test Procedure to prevent this occurrence.

\section{Assay Related}

S. The performance of vaginal swab specimens has not been evaluated in pregnant women.

T. The performance of endocervical, vaginal, and male urethral swab specimens, male and female urine specimens, and PreservCyt Solution liquid Pap specimens has not been evaluated in adolescents less than 16 years of age.

U. Do not use this kit after its expiration date.

V. Do not interchange, mix, or combine assay reagents from kits with different lot numbers. Aptima controls and assay fluids can be from different lot numbers.

\section{DTS Systems Specific}

W. Tips with hydrophobic plugs must be used. A minimum of two repeat pipettors must be dedicated for use with this assay: one for use in the target capture and amplification steps, and one for use in the HPA steps. Two micropipettors must be dedicated for use in this assay: one for use in specimen transfer and one for use in reagent preparation. All pipettors must be cleaned regularly as described in DTS Systems Test Procedure, Procedural Notes.

$X$. When using repeat pipettors for reagent addition, do not touch the tube with the pipette tip to prevent carryover from one tube to another.

Y. Adequate mixing is necessary to achieve accurate assay results. For complete details, see DTS Systems Test Procedure, Procedural Notes.

Z. Separate water baths must be dedicated for the target capture, amplification, and HPA steps in the assay.

AA.Assay reproducibility was established using spiked swab transport medium with rRNA. Reproducibility when testing swab and urine specimens containing target organism has not been determined.

AB.Sealing cards should be disposed of in the waste container immediately after removing them from reaction tubes. Fresh sealing cards should always be used: they should never 
be re-used from a previous step. Sealing cards should be firmly fixed to the top of all reaction tubes.

\section{Reagent Storage and Handling Requirements}

Note: For information on any hazard and precautionary statements that may be associated with reagents, refer to the Safety Data Sheet Library at www.hologic.com/sds.

A. The following reagents are stable when stored at $2^{\circ} \mathrm{C}$ to $8^{\circ} \mathrm{C}$ (refrigerated):

Aptima Amplification Reagent CT

Aptima Enzyme Reagent

Aptima Probe Reagent CT

Aptima Target Capture Reagent B

Aptima Positive Control, CT / Negative Control, GC

Aptima Positive Control, GC / Negative Control, CT

B. The following reagents are stable when stored at $2^{\circ} \mathrm{C}$ to $30^{\circ} \mathrm{C}$ :

Aptima Amplification Reconstitution Solution CT

Aptima Enzyme Reconstitution Solution

Aptima Probe Reconstitution Solution CT

Aptima Selection Reagent

C. The following reagents are stable when stored at $15^{\circ} \mathrm{C}$ to $30^{\circ} \mathrm{C}$ (room temperature):

Target Capture Reagent CT

Aptima Wash Solution

Aptima Buffer for Deactivation Fluid

Aptima Oil Reagent

D. Working Target Capture Reagent CT (wTCR CT) is stable for 60 days when stored at $15^{\circ} \mathrm{C}$ to $30^{\circ} \mathrm{C}$. Do not refrigerate.

E. After reconstitution, the Enzyme Reagent, Amplification Reagent CT, and Probe Reagent $\mathrm{CT}$ are stable for 60 days when stored at $2^{\circ} \mathrm{C}$ to $8^{\circ} \mathrm{C}$.

F. Discard any unused reconstituted reagents and wTCR CT after 60 days or after the Master Lot expiration date, whichever comes first.

G. Controls are stable until the date indicated on the vials.

H. Reagents from 100-test bottles stored on-board the Tigris DTS System have 96 hours of on-board stability.

I. The Probe Reagent CT and Reconstituted Probe Reagent CT are photosensitive. Store the reagents protected from light. 
J. Upon warming to room temperature, some control tubes may appear cloudy or contain precipitates. Cloudiness or precipitation associated with controls does not affect control performance. The controls may be used whether they are clear or cloudy/precipitated. If clear controls are desired, solubilization may be expedited by incubating them at the upper end of the room temperature range $\left(15^{\circ} \mathrm{C}\right.$ to $\left.30^{\circ} \mathrm{C}\right)$.

\section{K. Do not freeze the reagents.}

\section{Specimen Collection and Storage}

The Aptima CT Assay is designed to detect the presence of CT in clinician-collected endocervical, vaginal and male urethral swab specimens, patient-collected vaginal swab specimens, female and male urine specimens, and PreservCyt Solution liquid Pap specimens. Performance with specimens other than those collected with the following specimen collection kits has not been evaluated:

- Aptima Unisex Swab Specimen Collection Kit for Endocervical and Male Urethral Swab Specimens

- Aptima Urine Collection Kit for Male and Female Urine Specimens

- Aptima Vaginal Swab Specimen Collection Kit

- Aptima Specimen Transfer Kit (for use with gynecological samples collected in PreservCyt Solution)

A. Instructions for Collection:

Refer to the appropriate specimen collection kit package insert for collection instructions.

B. Specimen transport and storage before testing:

1. Swab specimens:

a. After collection, transport and store the swab in the swab specimen transport tube at $2^{\circ} \mathrm{C}$ to $30^{\circ} \mathrm{C}$ until tested. Specimens must be assayed with the Aptima CT Assay within 60 days of collection. If longer storage is needed, freeze at $-20^{\circ} \mathrm{C}$ to $-70^{\circ} \mathrm{C}$ for up to 12 months after collection (see Specimen Stability Studies).

2. Urine Specimens:

a. Urine samples that are still in the primary collection container must be transported to the lab at $2^{\circ} \mathrm{C}$ to $30^{\circ} \mathrm{C}$. Transfer the urine sample into the Aptima urine specimen transport tube within 24 hours of collection. Store at $2^{\circ} \mathrm{C}$ to $30^{\circ} \mathrm{C}$ and test within 30 days of collection.

b. After collection, transport the processed urine specimens in the Aptima urine specimen transport tube at $2^{\circ} \mathrm{C}$ to $30^{\circ} \mathrm{C}$ and store at $2^{\circ} \mathrm{C}$ to $30^{\circ} \mathrm{C}$ until tested. Processed urine specimens should be assayed with the Aptima CT Assay within 30 days of collection. If longer storage is needed, freeze at $-20^{\circ} \mathrm{C}$ to $-70^{\circ} \mathrm{C}$ for up to 12 months after collection (see Specimen Stability Studies).

3. PreservCyt Solution Liquid Pap Specimens:

a. PreservCyt Solution liquid Pap specimens intended for CT testing must be processed for cytology and/or transferred to an Aptima Specimen Transfer tube within 30 days of collection when stored at $2^{\circ} \mathrm{C}$ to $30^{\circ} \mathrm{C}$ (see Specimen Stability Studies).

b. If the ThinPrep Aliquot Removal procedure will be used, refer to the ThinPrep 2000 or ThinPrep 3000 Processor Operator's Manual-Addendum for instructions on aliquot removal. Transfer $1 \mathrm{~mL}$ of the removed aliquot into an Aptima 
Specimen Transfer tube according to the instructions in the Aptima Specimen Transfer Kit package insert.

c. If testing the specimen after processing using the ThinPrep 2000 Processor, process the PreservCyt Solution liquid Pap specimen in accordance with the ThinPrep 2000 Processor Operator's Manual and the Aptima Specimen Transfer

Kit package insert. Transfer $1 \mathrm{~mL}$ of the fluid remaining in the PreservCyt Solution vial into an Aptima Specimen Transfer tube according to the instructions in the Aptima Specimen Transfer Kit package insert.

d. Once the PreservCyt Solution liquid Pap specimen is transferred to the Aptima Specimen Transfer tube, the specimen must be assayed with the Aptima CT Assay within 30 days when stored at $2^{\circ} \mathrm{C}$ to $8^{\circ} \mathrm{C}$ or 14 days when stored at $15^{\circ} \mathrm{C}$ to $30^{\circ} \mathrm{C}$. If longer storage is needed, freeze at $-20^{\circ} \mathrm{C}$ to $-70^{\circ} \mathrm{C}$ for up to 12 months after transfer (see Specimen Stability Studies).

C. Specimen storage after testing:

1. Specimens that have been assayed must be stored upright in a rack.

2. The specimen transport tubes should be covered with a new, clean plastic film or foil barrier.

3. If assayed samples need to be frozen or shipped, remove the penetrable caps and place new non-penetrable or penetrable caps on the specimen transport tubes. If specimens need to be shipped for testing at another facility, recommended temperatures must be maintained. Prior to uncapping previously tested and recapped samples, specimen transport tubes must be centrifuged for 5 minutes at 420 RCF (Relative Centrifugal Force) to bring all of the liquid down to the bottom of the tube. Avoid splashing and cross-contamination.

Note: Specimens must be shipped in accordance with applicable national and international transportation regulations. 


\section{DTS Systems}

Reagents for the Aptima Assay for CT are listed below for the DTS Systems. Reagent Identification Symbols are also listed next to the reagent name.

\section{Reagents and Materials Provided}

Note: For information on any hazard and precautionary statements that may be associated with reagents, refer to the Safety Data Sheet Library at www.hologic.com/sds.

Aptima Chlamydia trachomatis Assay Kit, 100 tests (2 boxes) (Cat. No. 301088)

Aptima Chlamydia trachomatis Assay Refrigerated Box (Box 1 of 2) (store at $2^{\circ} \mathrm{C}$ to $8^{\circ} \mathrm{C}$ upon receipt)

\begin{tabular}{|c|c|c|}
\hline Symbol & Component & Quantity \\
\hline A & $\begin{array}{l}\text { Aptima Amplification Reagent CT } \\
\text { Non-infectious nucleic acids dried in buffered solution } \\
\text { containing }<5 \% \text { bulking agent. }\end{array}$ & 1 vial \\
\hline $\mathbf{E}$ & $\begin{array}{l}\text { Aptima Enzyme Reagent } \\
\text { Reverse transcriptase and RNA polymerase dried in HEPES } \\
\text { buffered solution containing }<10 \% \text { bulking reagent. }\end{array}$ & 1 vial \\
\hline $\mathbf{P}$ & $\begin{array}{l}\text { Aptima Probe Reagent CT } \\
\text { Non-infectious chemiluminescent DNA probes dried in } \\
\text { succinate buffered solution containing }<5 \% \text { detergent. }\end{array}$ & 1 vial \\
\hline TCR-B & $\begin{array}{l}\text { Aptima Target Capture Reagent B } \\
\text { Non-infectious nucleic acids in buffered solution containing } \\
<5 \% \text { detergent. }\end{array}$ & $1 \times 0.35 \mathrm{~mL}$ \\
\hline PCT/NGC & $\begin{array}{l}\text { Aptima Positive Control, CT I Negative Control, GC } \\
\text { Non-infectious CT nucleic acid in a buffered solution } \\
\text { containing }<5 \% \text { detergent. Each } 400 \mu \mathrm{L} \text { sample contains the } \\
\text { estimated rRNA equivalent of } 1 \text { CT IFU (5 fg/assay*). }\end{array}$ & $3 \times 1.7 \mathrm{~mL}$ \\
\hline PGC/NCT & $\begin{array}{l}\text { Aptima Positive Control, GC I Negative Control, CT } \\
\text { Non-infectious GC nucleic acid in a buffered solution } \\
\text { containing }<5 \% \text { detergent. Each } 400 \mu \mathrm{L} \text { sample contains the } \\
\left.\text { estimated rRNA equivalent of } 50 \mathrm{GC} \text { cells ( } 250 \text { fg/assay }{ }^{\star}\right) \text {. }\end{array}$ & $3 \times 1.7 \mathrm{~mL}$ \\
\hline
\end{tabular}

*The rRNA equivalents were calculated based on the genome size and estimated DNA:RNA ratio/cell of each organism.

Also included in the refrigerated box are the following (Storage Tray) (store at $2^{\circ} \mathrm{C}$ to $30^{\circ} \mathrm{C}$ upon receipt)

\begin{tabular}{clc} 
Symbol & Component & Quantity \\
\hline AR & $\begin{array}{l}\text { Aptima Amplification Reconstitution Solution CT } \\
\text { Aqueous solution containing preservatives. }\end{array}$ & $1 \times 9.3 \mathrm{~mL}$ \\
& Aptima Enzyme Reconstitution Solution & $1 \times 3.3 \mathrm{~mL}$ \\
& HEPES buffered solution containing a surfactant and glycerol. & \\
\hline PR & $\begin{array}{l}\text { Aptima Probe Reconstitution Solution CT } \\
\text { Succinate buffered solution containing }<5 \% \text { detergent. }\end{array}$ & $1 \times 12.4 \mathrm{~mL}$ \\
&
\end{tabular}


Also included in the refrigerated box are the following (Storage Tray) (continued) (store at $2^{\circ} \mathrm{C}$ to $30^{\circ} \mathrm{C}$ upon receipt)

\begin{tabular}{clc}
\hline Symbol & Component & Quantity \\
\hline $\mathbf{S}$ & $\begin{array}{l}\text { Aptima Selection Reagent } \\
600 \mathrm{mM} \text { borate buffered solution containing surfactant. }\end{array}$ & $1 \times 31 \mathrm{~mL}$ \\
\hline & Reconstitution Collars & 3 \\
\hline & Sealing Cards & 1 package \\
\hline
\end{tabular}

Aptima Chlamydia trachomatis Assay Room Temperature Box (Box 2 of 2) (store at $15^{\circ} \mathrm{C}$ to $30^{\circ} \mathrm{C}$ upon receipt)

\begin{tabular}{clc} 
Symbol & Component & Quantity \\
\hline TCR & $\begin{array}{l}\text { Aptima Target Capture Reagent CT } \\
\text { Buffered salt solution containing solid phase and capture } \\
\text { oligomers. }\end{array}$ & $1 \times 22 \mathrm{~mL}$ \\
\hline $\mathbf{W}$ & $\begin{array}{l}\text { Aptima Wash Solution } \\
10 \mathrm{mM} \text { HEPES buffered solution containing }<2 \% \text { detergent. }\end{array}$ & $1 \times 402 \mathrm{~mL}$ \\
\hline DF & $\begin{array}{l}\text { Aptima Buffer for Deactivation Fluid } \\
800 \mathrm{mM} \text { bicarbonate buffered solution. }\end{array}$ & $1 \times 402 \mathrm{~mL}$ \\
\hline O & $\begin{array}{l}\text { Aptima Oil Reagent } \\
\text { Silicone Oil. }\end{array}$ & $1 \times 24.6 \mathrm{~mL}$ \\
\hline
\end{tabular}

\section{Materials Required But Available Separately}

Note: Materials available from Hologic have catalog numbers listed, unless otherwise specified.

\begin{tabular}{|c|c|c|}
\hline Leader HC+ Luminometer & & $104747-01$ \\
\hline Hologic $^{\circledR}$ Target Capture System (TCS) & & 104555 \\
\hline Incubators and vortexers: & & - \\
\hline 2 Multi-tube vortex mixers & 102160 & \\
\hline $\begin{array}{l}3 \text { Circulating water baths } \\
\quad\left(62^{\circ} \mathrm{C} \pm 1^{\circ} \mathrm{C}, 42^{\circ} \mathrm{C} \pm 1^{\circ} \mathrm{C}, 62^{\circ} \mathrm{C} \pm 1^{\circ} \mathrm{C}\right)\end{array}$ & 104586 & \\
\hline 3 Water bath spacers & 104627 & \\
\hline OR & & \\
\hline $\begin{array}{l}2 \text { SB100 Dry Heat Bath/Vortexers } \\
\text { Additional SB100 baths may be required as }\end{array}$ & $\begin{array}{l}105524 \\
\text { e increases }\end{array}$ & \\
\hline Aptima Auto Detect Kit & & 301048 \\
\hline 2 eppendorf Repeater Plus pipettors & & 105725 \\
\hline 2 pipettors, $1000 \mu$ L RAININ PR1000 & & 901715 \\
\hline eppendorf pipettor, $20 \mu \mathrm{L}$ to $200 \mu \mathrm{L}$ & & 105726 \\
\hline Repeat pipettor tips, $2.5 \mathrm{~mL}$ & & $21-381-329$ \\
\hline Repeat pipettor tips, $5.0 \mathrm{~mL}$ & & $21-381-330$ \\
\hline
\end{tabular}


Cat. No.

Repeat pipettor tips, $25.0 \mathrm{~mL}$

21-381-115

Tips, P1000 Style

105049

special diameter tip only available from Hologic

Pipette tips $20 \mu \mathrm{L}$ to $200 \mu \mathrm{L}$

705512 (Fisher)

Ten Tube Units (TTU)

TU0022

Ten Tip Cassettes (TTC)

104578

Aptima Unisex Swab Specimen Collection Kit for Endocervical and 301041 Male Urethral Swab Specimens

Aptima Urine Specimen Collection Kit for Male and Female Urine 301040 Specimens

Aptima Urine Specimen Transport Tubes for Male and Female 105575 Urine Specimens

Aptima Vaginal Swab Specimen Collection Kit

301162

Aptima Specimen Transfer Kit

$301154 \mathrm{C}$

SysCheck calibration standard

301078

Bleach, $5 \%$ to $7 \%(0.7 \mathrm{M}$ to $1.0 \mathrm{M})$ sodium hypochlorite solution

Standard urine collection containers, without preservatives

Large-capped plastic container

Aptima penetrable caps

105668

Replacement non-penetrable caps

103036A

\section{Optional Materials}

Aptima Controls Kit

Aptima Assay Fluids

Aptima Wash Solution, Aptima Buffer for Deactivation Fluid, and Aptima Oil Reagent

Hologic Bleach Enhancer for Cleaning

for routine cleaning of surfaces and equipment

STD Proficiency Panel

Tips, $1000 \mu \mathrm{L}$ conductive, liquid sensing

TECAN Freedom EVO 100/4 containing

DTS 800 Systems Aptima Combo 2 Deck Plate

Reagent reservoir (40 $\mathrm{mL}$ quarter module)

Split reagent reservoir (19 $\mathrm{mL} \times 2$ quarter module)
105200

104765

104763
Cat. No.

301110

$302002 C$

302101

102325

10612513 (TECAN)

900932 


\section{DTS Systems Test Procedure}

A. Equipment Preparation

1. Adjust one water bath to $62^{\circ} \mathrm{C} \pm 1^{\circ} \mathrm{C}$ (for target capture, and primer annealing), a second water bath to $42^{\circ} \mathrm{C} \pm 1^{\circ} \mathrm{C}$ (for amplification), and a third water bath to $62^{\circ} \mathrm{C} \pm 1^{\circ} \mathrm{C}$ (for HPA). If using the SB $100^{\circledR}$ Dry Heat Bath/Vortexer, refer to the SB100 Dry Heat Bath/Vortexer Application Sheet (SB100 Application Sheet).

2. Prior to starting the assay, wipe down work surfaces and pipettors with $2.5 \%$ to $3.5 \%$ $(0.35 \mathrm{M}$ to $0.5 \mathrm{M})$ sodium hypochlorite solution. Allow the sodium hypochlorite solution to contact surfaces and pipettors for at least 1 minute, then follow with a water rinse. Do not allow the sodium hypochlorite solution to dry. Cover the bench surface on which the test will be performed with clean, plastic-backed, absorbent laboratory bench covers.

3. Place a sufficient number of Ten Tip Cassettes into the Target Capture System (TCS). Ensure that the TCS wash bottle is filled with Aptima Wash Solution and the aspiration manifold is connected to the vacuum pump. (Refer to the Target Capture System Operator's Manual.)

B. Reagent Reconstitution

Note: Reagent reconstitution should be performed prior to beginning specimen transfer.

1. To reconstitute Amplification CT, Enzyme, and Probe CT Reagents, combine the bottles of lyophilized reagent with the reconstitution solution. If refrigerated, allow the reconstitution solutions to reach room temperature before use.

a. Pair the appropriate reconstitution solution with the lyophilized reagent. The labels are color coded so that they can be paired correctly.

b. Open the lyophilized reagent vial and firmly insert the notched end of the reconstitution collar into the vial opening (Figure 1, Step 1).

c. Open the matching reconstitution solution bottle, and set the cap on a clean, covered work surface.

d. While holding the reconstitution solution bottle on the bench, firmly insert the other end of the reconstitution collar into the bottle opening (Figure 1, Step 2).

e. Slowly invert the assembled bottle and vial. Allow the solution to drain from the bottle into the vial (Figure 1, Step 3).

f. Gently swirl the solution in the vial to mix. Avoid creating foam while swirling the vial (Figure 1, Step 4).

g. Wait for the lyophilized reagent to go into solution, then invert the assembled bottle and vial again, tilting at a $45^{\circ}$ angle to minimize foaming (Figure 1, Step 5). Allow all of the liquid to drain back into the bottle.

h. Remove the reconstitution collar from the bottle (Figure 1, Step 6).

i. Recap the bottle. Record operator initials and reconstitution date on the label (Figure 1, Step 7). 
j. Discard the reconstitution collar and vial (Figure 1, Step 8).
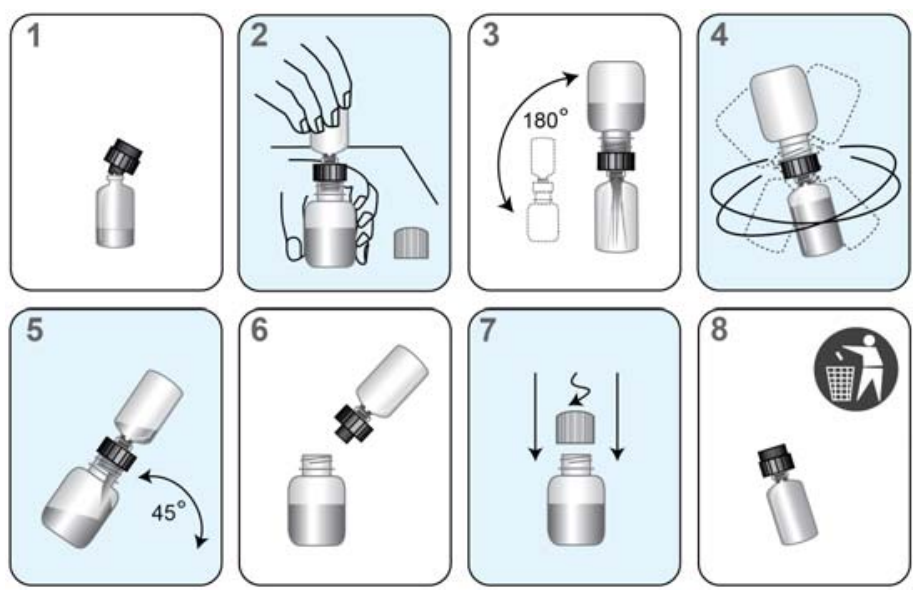

Figure 1. DTS Systems Reconstitution Process

2. Previously reconstituted Probe CT, Amplification CT, and Enzyme Reagents must reach room temperature $\left(15^{\circ} \mathrm{C}\right.$ to $\left.30^{\circ} \mathrm{C}\right)$ prior to the start of the assay. If Probe Reagent contains precipitate that does not return to solution at room temperature, heat at $62^{\circ} \mathrm{C}$ for 1 to 2 minutes. After this heat step, the Probe Reagent may be used even if residual precipitate remains. After resuspension, mix by gentle inversion, being careful not to induce foam.

Note: This inversion step should be performed any time that the precipitate is being brought into solution, whether by heating at $62^{\circ} \mathrm{C}$ or by warming at room temperature.

3. Prepare Working Target Capture Reagent CT (wTCR CT)

a. Transfer $20 \mathrm{~mL}$ of TCR CT to an appropriately sized, dedicated, clean, dry container.

b. Using a micropipettor, add $200 \mu \mathrm{L}$ of TCR-B into the TCR CT.

c. Thoroughly mix the solution by swirling.

d. Label the container. Record operator initials, preparation date, and both lot numbers.

Note: For a smaller number of reactions (specimens and controls), use the following to calculate volumes of TCR CT and TCR-B:

Volume of TCR CT $(\mathrm{mL})=$ (number of reactions +5 extra reactions) $\times 0.1 \mathrm{~mL}$

Volume of TCR-B $(\mathrm{mL})=$ Volume of TCR CT $(\mathrm{mL}) / 100$

C. Target Capture

The repeat pipettor used in target capture and amplification should be dedicated for use in these steps only. See Warnings and Precautions for more information.

\section{Rack Setup}

1. Allow the controls and specimens to reach room temperature prior to processing.

2. Do not vortex specimens.

3. Visually confirm that each specimen tube meets one of the following criteria:

a. The presence of a single blue Aptima collection swab in a unisex swab specimen transport tube. 
b. The presence of a single pink Aptima collection swab in a vaginal swab specimen transport tube.

c. A final volume of urine between the black fill lines of a urine specimen transport tube.

d. The absence of a swab in the Aptima specimen transport tube for PreservCyt Solution liquid Pap specimens.

4. Inspect specimen tubes before piercing them:

a. If a specimen tube contains bubbles in the space between the liquid and the cap, centrifuge the tube for 5 minutes at 420 RCF to eliminate the bubbles.

b. If a specimen tube has a lower volume than typically observed when collection instructions have been followed, centrifuge the tube for 5 minutes at $420 \mathrm{RCF}$ to ensure that no liquid is in the cap.

c. If the liquid level in a urine specimen tube is not between the two black indicator lines on the label, the specimen must be rejected. Do not pierce an overfilled tube.

d. If a urine specimen tube contains precipitate, heat the specimen at $37^{\circ} \mathrm{C}$ for up to 5 minutes. If the precipitate does not go back into solution, visually ensure that the precipitate does not prevent delivery of the specimen.

Note: Failure to follow Steps 4a-c may result in liquid discharge from the transport tube cap.

5. If specimens with standard caps (non-penetrable caps) are to be tested, they must be centrifuged for 5 minutes at 420 RCF (Relative Centrifugal Force) to bring all of the liquid down to the bottom of the tube before uncapping. Avoid splashing and crosscontamination.

6. In the Ten Tube Unit (TTU) rack, place enough TTUs to accommodate the controls and specimens.

7. If a worklist is desired, create the worklist at this point. For instructions on creating a worklist, refer to the Aptima Assay Software Operator's Manual.

8. Thoroughly mix the wTCR CT. Using the repeat pipettor, add $100 \mu \mathrm{L}$ into each reaction tube.

9. The first reaction tube of the assay must contain the negative control, and the second reaction tube must contain the positive control.

a. The negative control label for the Aptima CT Assay is blue-green. The label text identifies the negative control as "CONTROL + GC PGC / CONTROL - CT NCT". The positive control label for the Aptima CT Assay is pink. The label text identifies the positive control as "CONTROL + CT PCT / CONTROL - GC NGC".

b. Hold the negative control tube (blue-green-labeled tube) in one hand or keep it in a rack. Using a micropipettor, pierce the cap, taking care not to drive the tip into the bottom of the tube. Add $400 \mu \mathrm{L}$ of the negative control (blue-green-labeled tube) to the first reaction tube. In the same manner and using a new pipette tip, add $400 \mu \mathrm{L}$ of the positive control (pink-labeled tube) to the second reaction tube.

10. Continue the rack setup procedure by adding $400 \mu \mathrm{L}$ of each specimen into the remaining reaction tubes. Use a new pipette tip for each specimen and control. The acceptable volume of specimen or control added to a reaction tube is $400 \mu \mathrm{L} \pm 100 \mu \mathrm{L}$. See Procedural Notes, Control and Specimen Pipetting for more information. 


\section{Target Capture}

Use of the Hologic Target Capture System is described in the Target Capture System Operator's Manual. If using the SB100 Dry Heat Bath/Vortexer, refer to the SB100 Application Sheet.

11. Cover the TTUs with sealing cards and shake the rack gently by hand. Do not vortex. Incubate the rack at $62^{\circ} \mathrm{C} \pm 1^{\circ} \mathrm{C}$ in a water bath for $30 \pm 5$ minutes.

12. Remove the rack from the water bath and blot the bottoms of the tubes dry on absorbent material.

13. Ensure the sealing cards are firmly seated. If necessary, replace them with new sealing cards and seal the TTUs tightly.

14. Vortex the rack for 60 seconds on the multi-tube vortex mixer. See Procedural Notes, Vortexing for details. Begin vortexing within 2 minutes of removal of the rack from the water bath.

15. Without removing the sealing cards, incubate the rack at room temperature for $30 \pm 5$ minutes.

16. Place the rack on the TCS magnetic base for 5 to 10 minutes.

17. Prime the dispense station pump line by pumping Aptima Wash Solution through the dispense manifold. Pump enough liquid through the system so that there are no air bubbles in the line and that all ten nozzles are delivering a steady stream of liquid.

18. Turn on the vacuum pump and disconnect the aspiration manifold at the first connector between the aspiration manifold and the trap bottle. Ensure that the vacuum gauge meets the leak test specification. ${ }^{2}$ It may take 15 seconds to achieve this reading. Reconnect the aspiration manifold, and ensure that the vacuum gauge meets the vacuum level specification. Leave the vacuum pump on until all target capture steps are completed and the aspiration manifold tubing is dry.

19. Firmly attach the aspiration manifold to the first set of tips. Aspirate all liquid by lowering the tips into the first TTU until the tips come into brief contact with the bottoms of the tubes. Do not hold the tips in contact with the bottoms of the tubes.

20. After the aspiration is complete, eject the tips into their original TTC. Repeat the aspiration steps for the remaining TTUs, using a dedicated tip for each specimen.

21. Place the dispense manifold over each TTU and, using the dispense station pump, deliver $1.0 \mathrm{~mL}$ of Aptima Wash Solution into each tube of the TTU.

22. Cover the tubes with a sealing card and remove the rack from the TCS magnetic base. Vortex the rack once on the multi-tube vortex mixer. See Procedural Notes, Vortexing for details.

23. Place the rack on the TCS magnetic base for 5 to 10 minutes.

24. Aspirate all liquid as in Steps 19 and 20.

25. After the final aspiration, remove the rack from the TCS magnetic base and visually inspect the tubes to ensure that all liquid has been aspirated, and all tubes contain magnetic particle pellets. If any liquid is visible, place the rack back onto the TCS magnetic base for 2 minutes and repeat the aspiration for that TTU using the same tips used previously for each specimen.

Note: If a magnetic particle pellet is visible after aspiration is completed, the tube may be accepted. If no pellet is visible, the specimen should be retested. If the same specimen does not contain a magnetic particle pellet at this step in a subsequent run, this may indicate a specimen-specific problem. Re-collection of the specimen is recommended in this situation.

2 See the Target Capture System Vacuum Specifications Sheet located at the back of the Target Capture System Operator's Manual or contact Technical Support. 
D. Amplification

If using the SB100 Dry Heat Bath/Vortexer, refer to the SB100 Application Sheet.

1. Using the repeat pipettor, add $75 \mu \mathrm{L}$ of the reconstituted Amplification Reagent $\mathrm{CT}$ to each reaction tube. All reaction mixtures in the rack should now be red.

2. Using the repeat pipettor, add $200 \mu \mathrm{L}$ of Oil Reagent to each reaction tube.

3. Cover the tubes with a sealing card and vortex them on the multi-tube vortex mixer.

4. Incubate the rack in a water bath at $62^{\circ} \mathrm{C} \pm 1^{\circ} \mathrm{C}$ for $10 \pm 5$ minutes.

5. Transfer the rack into a water bath at $42^{\circ} \mathrm{C} \pm 1^{\circ} \mathrm{C}$ and incubate for $5 \pm 2$ minutes.

6 . With the rack in the water bath, carefully remove the sealing card and, using the repeat pipettor, add $25 \mu \mathrm{L}$ of the reconstituted Enzyme Reagent to each reaction tube. All reaction mixtures should now be orange.

7. Immediately cover the tubes with a fresh sealing card, remove the rack from the water bath, and mix the reaction tubes by gently shaking the rack by hand.

8. Incubate the rack in a water bath at $42^{\circ} \mathrm{C} \pm 1^{\circ} \mathrm{C}$ for $60 \pm 15$ minutes.

E. Hybridization Protection Assay (HPA)

If using the SB100 Dry Heat Bath/Vortexer, refer to the SB100 Application Sheet.

The repeat pipettor used in the hybridization and selection steps should be dedicated for use in these steps only. See Warnings and Precautions.

1. Hybridization

a. Remove the rack from the water bath and transfer it to the HPA area. Using the repeat pipettor, add $100 \mu \mathrm{L}$ of the reconstituted Probe Reagent CT to each reaction tube. All reaction mixtures should now be yellow.

b. Cover the tubes with a sealing card and vortex the rack on the multi-tube vortex mixer.

c. Incubate the rack in a $62^{\circ} \mathrm{C} \pm 1^{\circ} \mathrm{C}$ water bath for $20 \pm 5$ minutes.

d. Remove the rack from the water bath and incubate it at room temperature for $5 \pm 1$ minutes.

2. Selection

a. Using the repeat pipettor, add $250 \mu \mathrm{L}$ of Selection Reagent to each reaction tube. All reaction mixtures should now be red.

b. Cover the tubes with a sealing card, vortex the rack for 10 seconds or until the color is uniform, and incubate the rack in a water bath at $62^{\circ} \mathrm{C} \pm 1^{\circ} \mathrm{C}$ for $10 \pm 1$ minutes.

c. Remove the rack from the water bath.

3. Detection

Detection must be performed at $18^{\circ} \mathrm{C}$ to $28^{\circ} \mathrm{C}$.

a. Incubate the rack at $18^{\circ} \mathrm{C}$ to $28^{\circ} \mathrm{C}$ for $15 \pm 3$ minutes.

Note: This temperature range is critical for assay performance.

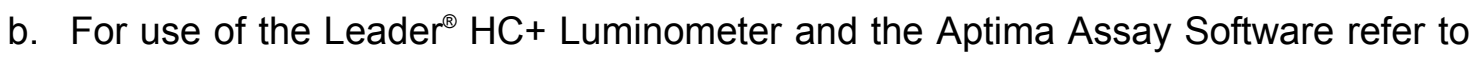
the Leader HC+ Luminometer Operator's Manual and the Aptima Assay Software Operator's Manual.

c. Ensure there are sufficient volumes of Auto Detect 1 and 2 to complete the tests. 
d. Prepare the Leader HC+ Luminometer by placing one empty TTU in cassette position number 1 and performing the Wash protocol.

e. Load the TTUs into the luminometer.

f. Log on to the computer. Click New Run, choose Aptima CT Assay Protocol and enter the number of tubes (controls and specimens). Click Next to begin the run.

Note: The run must be completed within 2 hours of the end of the selection step incubation.

g. Prepare Deactivation Fluid by mixing equal volumes of $5 \%$ to $7 \%(0.7 \mathrm{M}$ to $1.0 \mathrm{M})$ sodium hypochlorite solution and Aptima Buffer for Deactivation Fluid in a largecapped plastic container. Label and write the expiration date on the plastic container. Deactivation Fluid is stable for 4 weeks at room temperature. Discard Deactivation Fluid after 4 weeks or after 100 processed samples have been deactivated (whichever comes first).

h. After removing the used TTUs from the luminometer, place the TTUs into the container of Deactivation Fluid. Allow the TTUs to sit in the container for 15 minutes before disposal. Proper handling and disposal methods should be established by the laboratory director.

\section{Procedural Notes}

A. Controls

To work properly with the Aptima Assay software, the Negative Control for CT, which is labeled "CONTROL + GC PGC / CONTROL - CT NCT," must be in the first position of the first TTU. The Positive Control for CT, which is labeled "CONTROL + CT PCT I CONTROL - GC NGC," must be in the second position of the first TTU. Placement in the wrong position will cause the run to fail. Any additional controls must be entered as patient specimens and monitored by the operator for acceptability. The Positive Control for GC serves as the Negative Control for the Aptima CT Assay.

B. Control and Specimen Pipetting

The volume of control or specimen added to the reaction tube should be $400 \mu \mathrm{L} \pm 100$ $\mu \mathrm{L}$. Visual inspection of the volume pipetted into the reaction tube is recommended to ensure proper volume transfer. Proper control or specimen volume is needed to provide accurate results. If the proper volume has not been pipetted, re-pipette the WTCR CT and the control or specimen into a new reaction tube.

C. Reagents

Probe Reconstitution Solution may precipitate during storage. If this occurs, heat the Probe Reconstitution Solution at $62^{\circ} \mathrm{C}$ for 1 to 2 minutes. After this heat step, the Probe Reconstitution Solution may be used even if residual precipitate remains. After resuspension, mix the vial by gentle inversion, being careful not to induce foam.

D. Temperature

1. The target capture, amplification, hybridization, and selection steps are temperature dependent. Therefore, it is imperative that the water baths be maintained within their specified temperature ranges.

2. Room temperature is defined as $15^{\circ} \mathrm{C}$ to $30^{\circ} \mathrm{C}$.

3. The detection steps in the assay must be carried out at $18^{\circ} \mathrm{C}$ to $28^{\circ} \mathrm{C}$. 
E. Time

The target capture, amplification, hybridization, and selection reactions are all time dependent. Adhere to the times specified in the DTS Systems Test Procedure.

F. Vortexing

Proper vortexing is important to the successful performance of the Aptima CT Assay. When adequate vortexing motion is achieved, the suspension rotates at a rate that raises the solution into the upper half of the tube. This manipulation (vortexing) is maintained for specified periods of time. To vortex reactions, set the multi-tube vortex mixer speed to the lowest setting, secure the rack, and turn on power. Slowly increase speed until the liquid rises halfway up the tube. Vortex for 10 seconds, the indicated amount of time, or until the color is uniform. Then, turn speed to lowest setting before turning off the multi-tube vortex mixer and removing the rack. The reaction mixtures should never touch the sealing cards.

G. Water Baths

1. The level of the water in the water baths must be maintained at 1.5 inches to 2.0 inches $(3.8 \mathrm{~cm}$ to $5 \mathrm{~cm}$ ) deep as measured from the supporting metal tray (on the bottom of the water bath) to the surface of the water. This will ensure proper heat transfer.

2. To avoid cross-contamination, water baths should be dedicated to a specific assay step.

H. Decontamination

1. Surfaces and Pipettors

Laboratory bench surfaces and pipettors must be decontaminated regularly with $2.5 \%$ to $3.5 \%(0.35 \mathrm{M}$ to $0.5 \mathrm{M})$ sodium hypochlorite solution. Allow sodium hypochlorite solution to contact surfaces for at least 1 minute and then follow with a water rinse. Do not allow the sodium hypochlorite solution to dry. Chlorine solutions may pit equipment and metal. Thoroughly rinse equipment with water to avoid pitting.

2. TCS Aspiration Manifold

a. Place a new TTC into the TTC rack. Turn on the vacuum pump. Attach the aspiration manifold to the tips in the TTC. Aspirate all Wash Solution remaining in the priming trough of the Wash Solution dispense station. (Move the dispense manifold out of the way.)

b. Pour at least $100 \mathrm{~mL}$ of $0.5 \%$ to $0.7 \%(0.07 \mathrm{M}$ to $0.1 \mathrm{M})$, or if preferred $2.5 \%$ to $3.5 \%(0.35 \mathrm{M}$ to $0.5 \mathrm{M})$, sodium hypochlorite solution into the priming trough. Aspirate all of the solution through the aspiration manifold.

c. Pour at least $100 \mathrm{~mL}$ of deionized water into the priming trough. Aspirate all of the water through the aspiration manifold.

d. Eject the tips into their original TTC.

e. Leave the vacuum pump on until the manifold tubing is dry to prevent back flow.

f. Decontaminate the aspiration manifold surfaces as described in TCS Unit.

3. TCS Waste Container

When the waste bottle is $25 \%$ full or weekly, remove the waste bottle from the Target Capture System.

a. Turn off the vacuum pump and allow the vacuum pressure to equalize. 
b. Release the quick disconnect fittings between the waste bottle and overflow bottle, and the waste bottle and aspiration manifold.

c. Remove the waste bottle from the vacuum trap enclosure.

d. Remove the cap and carefully add $400 \mathrm{~mL}$ of $5 \%$ to $7 \%(0.7 \mathrm{M}$ to $1.0 \mathrm{M})$ sodium hypochlorite solution to the bottle (or $1 \mathrm{~L}$ if using a $10 \mathrm{~L}$ waste bottle).

Note: This may be done in a fume hood to avoid the release of fumes into the laboratory.

e. Cap the waste bottle and gently swirl the contents until fully mixed.

f. Let the waste bottle sit for 15 minutes and then dispose of the contents (waste).

g. Rinse the waste bottle with water to remove any remaining waste.

h. Cap the empty waste bottle and place it in the vacuum trap enclosure. Attach the quick disconnect fitting to the TCS unit. Carefully discard both gloves.

4. TCS Unit

Wipe the surfaces of the TCS unit, aspiration manifold, and wash buffer ejector tips with paper towels moistened with $2.5 \%$ to $3.5 \%(0.35 \mathrm{M}$ to $0.5 \mathrm{M})$ sodium hypochlorite solution. Follow the sodium hypochlorite solution step with a water rinse and then dry the surfaces completely with paper towels.

5. Racks

Submerge the racks in $2.5 \%$ to $3.5 \%(0.35 \mathrm{M}$ to $0.5 \mathrm{M})$ sodium hypochlorite solution, ensuring that they are covered by the sodium hypochlorite solution. Keep the racks submerged for 10 minutes. Longer exposure could damage the racks. Rinse the racks thoroughly with water, place the racks on a clean absorbent pad, and allow the racks to air-dry thoroughly. To prolong the life of the racks, allow the racks to dry upright, not upside-down.

I. Assay Contamination

1. The introduction of contaminating materials may occur if sufficient care is not taken during the assay protocol.

2. TTUs must be decontaminated in Deactivation Fluid as described under Detection. Do not reuse the TTUs.

3. Perform regular decontamination of equipment and work surfaces as described in Procedural Notes, Decontamination.

4. As in any reagent system, excess powder on some gloves may cause contamination of opened tubes. Powderless gloves are recommended.

J. Lab Contamination Monitoring Protocol for DTS Systems

There are many laboratory-specific factors that may contribute to contamination, including testing volume, workflow, disease prevalence and various other laboratory activities. These factors should be taken into consideration when contamination monitoring frequency is being established. Intervals for contamination monitoring should be established based on each laboratory's practices and procedures. 
To monitor for laboratory contamination, the following procedure may be performed using the Aptima Unisex Swab Specimen Collection Kit for Endocervical and Male Urethral Swab Specimens:

1. Label swab transport tubes with numbers corresponding to the areas to be tested.

2. Remove the specimen collection swab (blue shaft swab with green printing) from its packaging, wet the swab in the swab transport medium, and swab the designated area using a circular motion.

3. Immediately insert the swab into a transport tube.

4. Carefully break the swab shaft at the score line; use care to avoid splashing of the contents.

5. Recap the swab transport tube tightly.

6. Repeat Steps 2 to 5 for each area to be swabbed.

7. Test the swab using the Aptima CT Assay according to the DTS Systems Test Procedure.

If the results are CT positive or equivocal (see Test Interpretation - QC/Patient Results), the surface may be contaminated and should be decontaminated by treating with sodium hypochlorite solution as recommended in DTS Systems Test Procedure, Equipment Preparation.

Note: If contamination of the water bath is suspected, the bath water can be tested using the urine specimen test procedure, by adding $2.0 \mathrm{~mL}$ of the water to a urine specimen transport tube.

K. Troubleshooting

1. Low positive control values may be caused by incorrect temperatures during various steps in the assay or by allowing the selection time in the selection step to go longer than the recommended time.

2. High backgrounds may occur if the selection time in the selection step is shortened, the selection temperature is not correct, or insufficient mixing occurs after the addition of the Selection Reagent.

3. If the Aptima positive control for GC, which is labeled "CONTROL + GC PGC / CONTROL - CT NCT", is positive or equivocal for CT, see Procedural Notes, Assay Contamination for more information. 


\section{Tigris DTS System}

Reagents for the Aptima CT Assay are listed below for the Tigris DTS System. Reagent Identification Symbols are also listed next to the reagent name.

\section{Reagents and Materials Provided}

Note: For information on any hazard and precautionary statements that may be associated with reagents, refer to the Safety Data Sheet Library at www.hologic.com/sds.

\section{Aptima Chlamydia trachomatis Assay Kit}

100 tests (2 boxes and 1 Controls kit) (Cat. No. 303091)

\section{Aptima Chlamydia trachomatis Assay Refrigerated Box (Box 1 of 2)} (store at $2^{\circ} \mathrm{C}$ to $8^{\circ} \mathrm{C}$ upon receipt)

\begin{tabular}{|c|c|c|}
\hline Symbol & Component & $\begin{array}{l}\text { Quantity } \\
100 \text { test kit }\end{array}$ \\
\hline A & $\begin{array}{l}\text { Aptima Amplification Reagent CT } \\
\text { Non-infectious nucleic acids dried in buffered } \\
\text { solution containing }<5 \% \text { bulking agent. }\end{array}$ & 1 vial \\
\hline$E$ & $\begin{array}{l}\text { Aptima Enzyme Reagent } \\
\text { Reverse transcriptase and RNA polymerase } \\
\text { dried in HEPES buffered solution containing } \\
<10 \% \text { bulking reagent. }\end{array}$ & 1 vial \\
\hline $\mathbf{P}$ & $\begin{array}{l}\text { Aptima Probe Reagent } \mathbf{C T} \\
\text { Non-infectious chemiluminescent DNA probes } \\
\text { dried in succinate buffered solution containing } \\
<5 \% \text { detergent. }\end{array}$ & 1 vial \\
\hline TCR-B & $\begin{array}{l}\text { Aptima Target Capture Reagent B } \\
\text { Non-infectious nucleic acids in a buffered } \\
\text { solution containing }<5 \% \text { detergent. }\end{array}$ & $1 \times 0.30 \mathrm{~mL}$ \\
\hline
\end{tabular}


Aptima Chlamydia trachomatis Assay Room Temperature Box (Box 2 of 2) (store at $15^{\circ} \mathrm{C}$ to $30^{\circ} \mathrm{C}$ upon receipt)

\begin{tabular}{|c|c|c|}
\hline \multirow{2}{*}{ Symbol } & \multirow{2}{*}{ Component } & Quantity \\
\hline & & 100 test kit \\
\hline \multirow[t]{2}{*}{ AR } & $\begin{array}{l}\text { Aptima Amplification Reconstitution } \\
\text { Solution CT }\end{array}$ & $1 \times 11.9 \mathrm{~mL}$ \\
\hline & Aqueous solution containing preservatives. & \\
\hline ER & $\begin{array}{l}\text { Aptima Enzyme Reconstitution Solution } \\
\text { HEPES buffered solution containing a } \\
\text { surfactant and glycerol. }\end{array}$ & $1 \times 6.3 \mathrm{~mL}$ \\
\hline PR & $\begin{array}{l}\text { Aptima Probe Reconstitution Solution CT } \\
\text { Succinate buffered solution containing }<5 \% \\
\text { detergent. }\end{array}$ & $1 \times 15.2 \mathrm{~mL}$ \\
\hline $\mathbf{S}$ & $\begin{array}{l}\text { Aptima Selection Reagent } \\
600 \mathrm{mM} \text { borate buffered solution containing } \\
\text { surfactant. }\end{array}$ & $1 \times 43.0 \mathrm{~mL}$ \\
\hline \multirow[t]{4}{*}{ TCR } & $\begin{array}{l}\text { Aptima Target Capture Reagent } \mathbf{C T} \\
\text { Buffered salt solution containing solid phase } \\
\text { and capture oligomers. }\end{array}$ & $1 \times 26.0 \mathrm{~mL}$ \\
\hline & Reconstitution Collars & 3 \\
\hline & Transfer Pipettes & \\
\hline & Master Lot Barcode Sheet & 1 sheet \\
\hline
\end{tabular}

\section{Aptima Controls Kit} (store at $2^{\circ} \mathrm{C}$ to $8^{\circ} \mathrm{C}$ upon receipt)

\begin{tabular}{cll} 
Symbol & Component & Quantity \\
\hline PCTI & Aptima Positive Control, CT I Negative Control, GC & $5 \times 1.7 \mathrm{~mL}$ \\
NGC & $\begin{array}{l}\text { Non-infectious CT nucleic acid in a buffered solution containing }<5 \% \\
\text { detergent. Each } 400 \mu L \text { sample contains the estimated rRNA } \\
\\
\text { equivalent of 1 CT IFU (5 fg/assay*). }\end{array}$ & \\
\hline PGCI & Aptima Positive Control, GC I Negative Control, CT & $5 \times 1.7 \mathrm{~mL}$ \\
NCT & $\begin{array}{l}\text { Non-infectious GC nucleic acid in a buffered solution containing }<5 \% \\
\text { detergent. Each 400 } \mu \text { L sample contains the estimated } r R N A\end{array}$ & \\
& equivalent of 50 GC cells (250 fg/assay*).
\end{tabular}

*The rRNA equivalents were calculated based on the genome size and estimated DNA:RNA ratio/cell of each organism. 


\section{Materials Required But Available Separately}

Note: Materials available from Hologic have catalog numbers listed, unless otherwise specified.

Cat. No.

Tigris DTS System

105118

Aptima Assay Fluids Kit

302382

(Aptima Wash Solution, Aptima Buffer for Deactivation Fluid, and Aptima Oil Reagent)

Aptima Auto Detect Kit

301048

Aptima System Fluid Preservative Kit

302380

Tips, $1000 \mu \mathrm{L}$ conductive, liquid sensing

10612513 (TECAN)

Tigris DTS System Run Kit containing

301191

Multi-tube Units (MTU)

MTU-Tiplet Waste Bag Kit

104772-02

MTU Waste Deflectors

900907

900931

MTU Waste Covers

105523

Aptima Specimen Transfer Kit

$301154 C$

for use with specimens in PreservCyt Solution

Aptima Vaginal Swab Specimen Collection Kit

301162

Aptima Unisex Swab Specimen Collection Kit for Endocervical and 301041 Male Urethral Swab Specimens

Aptima Urine Specimen Collection Kit for Male and Female Urine 301040 Specimens

Aptima Urine Specimen Transport Tubes for Male and Female Urine Specimens

Bleach, $5 \%$ to $7 \%(0.7 \mathrm{M}$ to $1.0 \mathrm{M})$ sodium hypochlorite solution

Water for the Tigris DTS System

consult the Tigris DTS System Operator's Manual for specifications

Disposable gloves

SysCheck calibration standard

301078

Aptima penetrable caps

105668

Replacement non-penetrable caps

103036A

Replacement caps for the 100-test kits

Amplification, Enzyme, and Probe reagent reconstitution solutions

TCR and Selection reagent

CL0041 (100 caps)

501604 (100 caps) 


\section{Optional Materials}

Aptima Controls Kit

Hologic Bleach Enhancer for Cleaning

for routine cleaning of surfaces and equipment
Cat. No.

301110

302101

\section{Tigris DTS System Test Procedure}

Note: See Tigris DTS System Operator's Manual for additional Tigris DTS System procedural information.

A. Work Area Preparation

Clean work surfaces where reagents and samples will be prepared. Wipe down work surfaces with $2.5 \%$ to $3.5 \%(0.35 \mathrm{M}$ to $0.5 \mathrm{M})$ sodium hypochlorite solution. Allow the sodium hypochlorite solution to contact surfaces for at least 1 minute and then follow with a water rinse. Do not allow the sodium hypochlorite solution to dry. Cover the bench surface on which the reagents and samples will be prepared with clean, plastic-backed absorbent laboratory bench covers.

B. Reagent Reconstitution/Preparation of a New Kit

Note: Reagent reconstitution should be performed prior to beginning any work on the Tigris DTS System.

1. To reconstitute Amplification CT, Enzyme, and Probe CT Reagents, combine the bottles of lyophilized reagent with the reconstitution solution. If refrigerated, allow the reconstitution solutions to reach room temperature before use.

a. Pair each reconstitution solution with its lyophilized reagent. Ensure that the reconstitution solution and lyophilized reagent have matching label colors before attaching the reconstitution collar.

b. Check the lot numbers on the Master Lot Barcode Sheet to ensure that the appropriate reagents are paired.

c. Open the lyophilized reagent vial and firmly insert the notched end of the reconstitution collar into the vial opening (Figure 2, Step 1).

d. Open the matching reconstitution solution bottle, and set the cap on a clean, covered work surface.

e. While holding the reconstitution solution bottle on the bench, firmly insert the other end of the reconstitution collar into the bottle opening (Figure 2, Step 2).

f. Slowly invert the assembled bottles. Allow the solution to drain from the bottle into the glass vial (Figure 2, Step 3).

g. Gently swirl the solution in the vial to mix. Avoid creating foam while swirling the vial (Figure 2, Step 4).

h. Wait for the lyophilized reagent to go into solution, then invert the assembled bottles again, tilting at a $45^{\circ}$ angle to minimize foaming (Figure 2, Step 5). Allow all of the liquid to drain back into the plastic bottle.

i. Remove the reconstitution collar and glass vial (Figure 2, Step 6).

j. Recap the bottle. Record operator initials and the reconstitution date directly on the label. 
k. Discard the reconstitution collar and glass vial (Figure 2, Step 8).

Warning: Avoid creating foam when reconstituting reagents. Foam compromises the level-sensing in the Tigris DTS System.
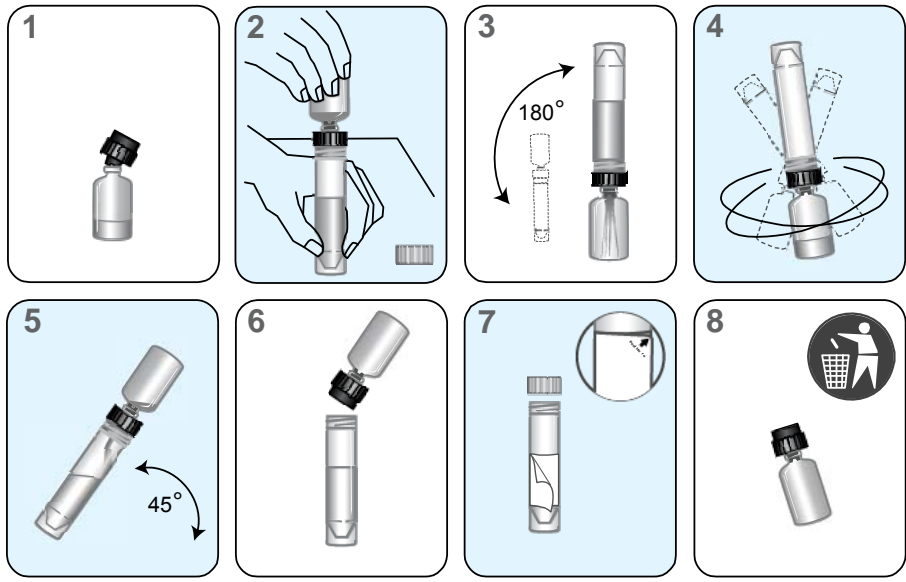

Figure 2. Tigris DTS System Reconstitution Process

2. Prepare working TCR CT (WTCR) for the 100-test kits

a. Pair the appropriate bottles of TCR CT and TCR-B.

b. Check the reagent lot numbers on the Master Lot Barcode Sheet to make sure that the appropriate reagents in the kit are paired.

c. Open the bottle of TCR CT and set the cap on a clean, covered work surface.

d. Open the bottle of TCR-B and pour the entire contents into the bottle of TCR CT. Expect a small amount of liquid to remain in the TCR-B bottle.

e. Cap the bottle of TCR CT and gently swirl the solution to mix the contents. Avoid creating foam during this step.

f. Record operator initials and the current date on the label.

g. Discard the TCR-B bottle and cap.

3. Prepare Selection Reagent

a. Check the lot number on the reagent bottle to make sure that it matches the lot number on the Master Lot Barcode Sheet.

b. Record operator initials and the current date on the label.

Note: Thoroughly mix by gently inverting all reagents prior to loading on the system. Avoid creating foam during inversion of reagents.

C. Reagent Preparation for Previously Reconstituted Reagents

1. Previously reconstituted Amplification CT, Enzyme, and Probe CT Reagents must reach room temperature $\left(15^{\circ} \mathrm{C}\right.$ to $\left.30^{\circ} \mathrm{C}\right)$ prior to the start of the assay.

2. If reconstituted Probe CT Reagent contains precipitate that does not return to solution at room temperature, heat the capped bottle at a temperature that does not exceed $62^{\circ} \mathrm{C}$ for 1 to 2 minutes. After this heat step, the Probe CT Reagent may be used even if residual precipitate remains. Mix Probe CT Reagent by inversion, being careful not to induce foam, prior to loading onto the system.

3. Thoroughly mix each reagent by gently inverting prior to loading on the system. Avoid creating foam during inversion of reagents. 
4. Do not top off reagent bottles. The Tigris DTS System will recognize and reject bottles that have been topped off.

D. Specimen Handling

1. Allow the controls and specimens to reach room temperature prior to processing.

2. Do not vortex specimens.

3. Visually confirm that each specimen tube meets one of the following criteria:

a. The presence of a single blue Aptima collection swab in a unisex swab specimen transport tube.

b. The presence of a single pink Aptima collection swab in a vaginal swab specimen transport tube.

c. A final volume of urine between the black fill lines of a urine specimen transport tube.

d. The absence of a swab in the Aptima specimen transport tube for PreservCyt Solution liquid Pap specimens.

4. Inspect specimen tubes before loading into rack:

a. If a specimen tube contains bubbles in the space between the liquid and the cap, centrifuge the tube for 5 minutes at 420 RCF to eliminate the bubbles.

b. If a specimen tube has a lower volume than typically observed when collection instructions have been followed, centrifuge the tube for 5 minutes at $420 \mathrm{RCF}$ to ensure that no liquid is in the cap.

c. If the liquid level in a urine specimen tube is not between the two black indicator lines on the label, the specimen must be rejected. Do not pierce an overfilled tube.

d. If a urine specimen tube contains precipitate, heat the specimen at $37^{\circ} \mathrm{C}$ for up to 5 minutes. If the precipitate does not go back into solution, visually ensure that the precipitate does not prevent delivery of the specimen.

Note: Failure to follow Steps 4a-c may result in liquid discharge from the specimen tube cap.

Note: Up to 3 separate aliquots can be tested from each specimen tube. Attempts to pipette more than 3 aliquots from the specimen tube can lead to insufficient volume errors.

\section{E. System Preparation}

Set up the system and worklist according to instructions in the Tigris DTS System Operator's Manual and Procedural Notes.

\section{Procedural Notes}

A. Controls

1. To work properly with the Tigris Aptima Assay software, front and end controls are required. The Positive Control, GC / Negative Control, CT must be in the first position and second to last position of a worklist. This control label is blue-green. The label text is "CONTROL + GC PGC / CONTROL - CT NCT". The Positive Control, CT / Negative Control, GC must be in the second position and last position of a worklist. This control label is pink. The label text is "CONTROL + CT PCT / CONTROL - GC NGC". 
2. Each Aptima control tube can be tested once. Attempts to pipette more than once from the tube can lead to insufficient volume errors.

B. Temperature

Room temperature is defined as $15^{\circ} \mathrm{C}$ to $30^{\circ} \mathrm{C}$.

C. Glove Powder

As in any reagent system, excess powder on some gloves may cause contamination of opened tubes. Powderless gloves are recommended.

D. Lab Contamination Monitoring Protocol for Tigris DTS System

There are many laboratory-specific factors that may contribute to contamination, including testing volume, workflow, disease prevalence and various other laboratory activities. These factors should be taken into consideration when contamination monitoring frequency is being established. Intervals for contamination monitoring should be established based on each laboratory's practices and procedures.

To monitor for laboratory contamination, the following procedure may be performed using the Aptima Unisex Swab Specimen Collection Kit for Endocervical and Male Urethral Swab Specimens:

1. Label swab transport tubes with numbers corresponding to the areas to be tested.

2. Remove the specimen collection swab (blue shaft swab with green printing) from its packaging, wet the swab in the swab transport medium, and swab the designated area using a circular motion.

3. Immediately insert the swab into a transport tube.

4. Carefully break the swab shaft at the score line; use care to avoid splashing of the contents.

5. Recap the swab transport tube tightly.

6. Repeat Steps 2 to 5 for each area to be swabbed.

If the results are CT positive or equivocal, see Test Interpretation - QC/Patient Results. For additional Tigris DTS System-specific contamination monitoring information, see the Tigris DTS System Operator's Manual. 


\section{Test Interpretation - QC/Patient Results}

A. Test Interpretation

Assay test results are automatically interpreted by the Aptima Assay Software using the CT protocol. A test result may be negative, equivocal, positive, or invalid as determined by total RLU in the detection step (see below). A test result may be invalid due to RLU values outside the normal expected ranges. Initial equivocal and invalid test results should be retested.

\begin{tabular}{cc}
\hline Test Interpretation & Total RLU $(\mathbf{x 1 0 0 0})$ \\
\hline Negative & $0^{*}$ to $<50$ \\
\hline Equivocal & 50 to $<100$ \\
\hline Low RLU Positive & 100 to $<5,000$ \\
\hline Positive $^{1,2}$ & 5,000 to $<12,000$ \\
\hline Invalid & $0^{*}$ or $>12,000$
\end{tabular}

* A zero $(0 \times 1000)$ RLU result on the run report represents a value between zero and $999 \mathrm{RLU}$. RLU values less than 160 on DTS Systems or 690 on Tigris DTS System will be reported as invalid.

${ }^{1}$ According to CDC guidelines, "consideration should be given to routine additional testing for persons with positive CT or GC screening tests when risk-factor information or actual surveys indicate that the prevalence is low, resulting in a lower PPV (e.g., < 90\%)." Refer to CDC guidelines for details on additional testing and patient management after a positive screening test (4).

${ }^{2}$ Refer to Table 3 for RLU distribution of results. The magnitude of RLU is not indicative of the level of organism in the specimen.

${ }^{3}$ In the low positive range, data suggest positive results should be interpreted carefully, with the understanding that the likelihood of a false positive may be higher than a true positive.

B. Quality Control Results and Acceptability

The Aptima Negative Control for CT, which is labeled "CONTROL + GC PGC I CONTROL - CT NCT," and the Aptima Positive Control for CT, which is labeled "CONTROL + CT PCT / CONTROL - GC NGC," act as controls for the target capture, amplification, and detection steps of the assay. In accordance with guidelines or requirements of local, state, and/or federal regulations or accrediting organizations, additional controls for cell lysis and RNA stabilization may be included. The Negative Control for CT, which is labeled "CONTROL + GC PGC / CONTROL - CT NCT," contains non-infectious GC rRNA. If desired, additional controls can be ordered as a kit. See Optional Materials. Correct preparation of specimens is confirmed visually by the presence of a single Aptima collection swab in a swab specimen transport tube, a final volume of urine in between the black fill lines of a urine specimen transport tube, or the absence of a swab in an Aptima specimen transfer tube for liquid Pap specimens.

The Aptima Assay Controls must produce the following test results:

\begin{tabular}{lcl}
\hline \multicolumn{1}{c}{ Control } & Total RLU $(\mathbf{x 1 0 0 0})$ & CT Result \\
\hline Positive Control, GC/ & $0^{*}$ and $<50$ & Negative \\
Negative Control, CT & $\geq 100$ and $<12,000$ & Positive \\
\hline Positive Control, CT/ & \\
Negative Control, GC &
\end{tabular}

* A zero $(0 \times 1000)$ RLU result on the run report represents a value between zero and 999 RLU. RLU values less than 160 on DTS Systems or 690 on Tigris DTS System will be reported as invalid. 
1. The Aptima Assay software automatically evaluates the controls according to the above criteria and will report the Run Status as PASS if the run control criteria are met, and FAIL if the run control criteria are not met.

2. If the Run Status is FAIL, all test results in the same run are invalid and must not be reported.

3. Each laboratory should implement appropriate control procedures to satisfy the requirements of CLIA regulations (section 493.1256).

Note: See Troubleshooting, or contact Hologic Technical Support for help with out-ofrange controls on the DTS Systems.

4. A Tigris DTS System parameter permits each site to specify a "control bracketing" frequency whereby additional sets of controls can be placed at defined intervals within the worklist. If this parameter is specified, the Tigris DTS System will require a set of controls to be placed after the defined number of specimens in the control bracket. The Tigris DTS System automatically evaluates each control in the worklist according to the above criteria and will invalidate all specimens in the affected control bracket(s) if the control criteria are not met. See the Tigris DTS System Operator's Manual for additional details.

5. Negative controls may not be effective in monitoring random carryover. See Tigris DTS System Analytical Performance for results from a high-target analytical carryover study that was performed to demonstrate control of carryover on the Tigris DTS System.

C. Specimen Preparation Control (optional)

The Aptima Negative Control for CT, which is labeled "CONTROL + GC PGC / CONTROL - CT NCT," and the Aptima Positive Control for CT, which is labeled "CONTROL + CT PCT / CONTROL - GC NGC," act as controls for the target capture, amplification, and detection steps of the assay and must be included in each assay run. If desired, controls for cell lysis and RNA stabilization can be tested in accordance with the requirements of appropriate accrediting organizations or individual laboratory procedures. Known positive specimens can serve as controls by being prepared and tested in conjunction with unknown specimens. Specimens used as preparation controls must be stored, handled, and tested according to the package insert. Specimen preparation controls should be interpreted in the same manner as described for patient test specimens. See Test Interpretation - QC/Patient Results, Patient Test Results.

D. Patient Test Results

1. If the controls in any run do not yield the expected results, test results on patient specimens in the same run must not be reported.

2. Swab, urine, and PreservCyt Solution liquid Pap specimen results. See Notes below. a. Initial results

\begin{tabular}{ll}
\hline CT Pos* & Positive for CT rRNA. \\
\hline CT Neg & Presumed negative for CT rRNA. \\
\hline CT Equiv & Sample should be retested. \\
\hline Invalid & Sample should be retested. \\
\hline
\end{tabular}


b. Retest results

\begin{tabular}{ll}
\hline CT Pos* & Positive for CT rRNA. \\
\hline CT Neg & Presumed negative for CT rRNA. \\
\hline CT Equiv & Indeterminate, a new specimen should be collected. \\
\hline Invalid & Indeterminate, a new specimen should be collected. \\
\hline
\end{tabular}

*Low RLU Positive specimen results are included in this category. See Test Interpretation above.

Notes

- The first valid, non-equivocal result for each analyte is the result that should be reported.

- Careful consideration of performance data is recommended for interpreting Aptima CT test results for asymptomatic individuals or any individuals in low prevalence populations.

- A negative result does not preclude the presence of a CT infection because results are dependent on adequate specimen collection, absence of inhibitors, and sufficient rRNA to be detected. Test results may be affected by improper specimen collection, improper specimen storage, technical error, specimen mix-up, or target levels below the assay limit of detection.

- Testing of an endocervical specimen is recommended for female patients who are clinically suspected of having a chlamydial or gonococcal infection. If both a Pap and endocervical swab are collected, the PreservCyt Solution liquid Pap specimen must be collected before the endocervical swab specimen. 


\section{Limitations}

A. Use of this assay is limited to personnel who have been trained in the procedure. Failure to follow the instructions given in this package insert may result in erroneous results.

B. The effects of tampon use, douching, and specimen collection variables have not been assessed for their impact on the detection of CT.

C. The presence of mucus in endocervical specimens does not interfere with the detection of CT by the Aptima CT Assay. However, to ensure collection of cells infected with CT, columnar epithelial cells lining the endocervix should be sampled. If excess mucus is not removed, sampling of these cells is not ensured.

D. Urine, vaginal swab, and PreservCyt Solution liquid Pap specimen sampling is not designed to replace cervical exams and endocervical specimens for diagnosis of female urogenital infections. Patients may have cervicitis, urethritis, urinary tract infections, or vaginal infections due to other causes or concurrent infections with other agents.

E. The Aptima CT Assay is not intended for the evaluation of suspected sexual abuse or for other medico-legal indications. For those patients for whom a false positive result may have adverse psycho-social impact, CDC recommends retesting by a method using an alternate technology (4).

F. Reliable results are dependent on adequate specimen collection. Because the transport system used for this assay does not permit microscopic assessment of specimen adequacy, training of clinicians in proper specimen collection techniques is necessary. Refer to package insert of the appropriate Aptima specimen collection kit.

G. Therapeutic failure or success cannot be determined with the Aptima CT Assay since nucleic acid may persist following appropriate antimicrobial therapy.

H. Results from the Aptima CT Assay should be interpreted in conjunction with other laboratory and clinical data available to the clinician.

I. A negative result does not preclude a possible infection because results are dependent on adequate specimen collection. Test results may be affected by improper specimen collection, technical error, specimen mix-up, or target levels below the assay limit of detection.

J. The Aptima CT Assay provides qualitative results. Therefore, a correlation cannot be drawn between the magnitude of a positive assay signal and the number of organisms in a specimen.

K. For the vaginal swab, endocervical swab, male urethral swab and urine specimen clinical studies, performance for detecting CT is derived from high prevalence populations. Positive results in low prevalence populations should be interpreted carefully with the understanding that the likelihood of a false positive may be higher than a true positive.

L. For the PreservCyt Solution liquid Pap specimen clinical studies, the Aptima CT Assay performance for detecting CT is derived primarily from low prevalence populations. Nonetheless, positive results in low prevalence populations should be interpreted carefully with the understanding that the likelihood of a false positive may be higher than a true positive. 
M. Performance of the Aptima Specimen Transfer kit was not evaluated for testing the same PreservCyt Solution liquid Pap specimen both before and after ThinPrep Pap processing.

N. PreservCyt Solution liquid Pap specimens processed with instruments other than the ThinPrep 2000 processor have not been evaluated for use in Aptima Assays.

O. Patient-collected vaginal swab specimens are an option for screening women when a pelvic exam is not otherwise indicated.

P. The patient-collected vaginal swab specimen application is limited to health care facilities where support/counseling is available to explain the procedures and precautions.

Q. The Aptima CT Assay has not been validated for use with vaginal swab specimens collected by patients at home.

R. Performance of the vaginal swab specimen has not been evaluated in pregnant women.

S. The performance of endocervical, vaginal, and male urethral swab specimens, male and female urine specimens, and PreservCyt Solution liquid Pap specimens has not been evaluated in adolescents less than 16 years of age.

T. The performance of the Tigris DTS System has not been determined at altitudes above 7355 feet $(2240 \mathrm{~m})$. Additional volumetric verifications and assay specific studies will be performed prior to, or as part of, the installation and acceptance process in laboratories above 7355 feet $(2240 \mathrm{~m})$ altitude.

U. There is no evidence of degradation of nucleic acids in PreservCyt Solution. If a PreservCyt Solution liquid Pap specimen has small numbers of CT cellular material, uneven distribution of this cellular material may occur. Also, when compared to direct sampling with the Aptima Swab Transport Media, the additional volume of PreservCyt Solution results in greater dilution of the sample material. These factors may affect the ability to detect small numbers of organisms in the collected material. If negative results from the specimen do not fit with the clinical impression, a new specimen may be necessary.

V. Customers must independently validate an LIS transfer process. 


\section{Clinical Study Results}

The performance of the Aptima CT Assay was established in two multi-center clinical investigations conducted in North America. In the first clinical investigation, two studies were conducted. First, the clinical specimen study established the sensitivity, specificity, and predictive values of the Aptima CT Assay using clinician-collected endocervical, vaginal, and male urethral swab specimens, patient-collected vaginal swab specimens, and male and female urine specimens. The second study, in the first clinical investigation, evaluated the precision of the Aptima CT Assay when performed according to NCCLS Guidelines (16). The second clinical investigation established the sensitivity, specificity, and predictive values of the Aptima CT Assay using PreservCyt Solution (component of the ThinPrep 2000 System). PreservCyt Solution liquid Pap specimens were also evaluated for within-laboratory precision with the Aptima CT Assay. 


\section{DTS Systems Expected Values}

\section{Prevalence}

The prevalence of CT in patient populations depends on risk factors such as age, gender, the presence of symptoms, the type of clinic, and the test method. A summary of the prevalence of CT, by specimen type as determined by the Aptima CT Assay is shown in Tables 1 and 1a for two multi-center clinical investigations by clinical site and overall.

Table 1: Prevalence of C. trachomatis by Clinical Site and Overall as Determined by Aptima CT Assay Results

\begin{tabular}{|c|c|c|c|c|c|c|c|c|c|c|c|c|}
\hline \multirow{3}{*}{$\begin{array}{c}\text { Site } \\
1\end{array}$} & \multicolumn{12}{|c|}{ \% (\#positive I \#tested) } \\
\hline & \multicolumn{2}{|r|}{ MS } & \multicolumn{2}{|r|}{ MU } & \multicolumn{2}{|r|}{ FS } & \multicolumn{2}{|r|}{ FU } & \multicolumn{2}{|r|}{ PVS } & \multicolumn{2}{|r|}{ CVS } \\
\hline & 27.0 & $(68 / 252)$ & 25.0 & $(63 / 252)$ & 16.5 & $(38 / 230)$ & 17.0 & $(39 / 229)$ & 19.2 & $(42 / 219)$ & 19.1 & $(44 / 230)$ \\
\hline 2 & 27.7 & $(98 / 354)$ & 26.6 & $(94 / 354)$ & 35.0 & $(70 / 200)$ & 26.5 & $(53 / 200)$ & 30.8 & $(61 / 198)$ & 33.0 & $(66 / 200)$ \\
\hline 3 & 25.0 & $(1 / 4)$ & 25.0 & $(1 / 4)$ & 11.4 & $(13 / 114)$ & 8.8 & $(10 / 113)$ & 10.8 & $(12 / 111)$ & 11.5 & $(13 / 113)$ \\
\hline 4 & N/A & N/A & N/A & N/A & 11.6 & $(31 / 267)$ & 8.1 & $(22 / 271)$ & 9.3 & $(25 / 268)$ & 12.2 & $(33 / 270)$ \\
\hline 5 & 8.0 & $(16 / 200)$ & 8.0 & $(16 / 200)$ & 9.0 & (18/199) & 7.5 & $(15 / 199)$ & 8.0 & $(16 / 199)$ & 10.1 & (20/199) \\
\hline 6 & 22.7 & $(69 / 304)$ & 20.0 & $(61 / 305)$ & 14.3 & $(42 / 294)$ & 13.2 & $(39 / 295)$ & 15.2 & $(44 / 290)$ & 16.2 & $(48 / 296)$ \\
\hline 7 & 5.8 & $(12 / 207)$ & 6.3 & $(13 / 207)$ & 7.8 & $(8 / 102)$ & 9.8 & $(10 / 102)$ & 12.7 & $(13 / 102)$ & 8.8 & $(9 / 102)$ \\
\hline 8 & N/A & $\mathrm{N} / \mathrm{A}$ & $\mathrm{N} / \mathrm{A}$ & N/A & 8.2 & $(4 / 49)$ & 6.1 & $(3 / 49)$ & 12.5 & $(6 / 48)$ & 7.8 & $(4 / 51)$ \\
\hline All & 20.0 & $(264 / 1321)$ & 18.8 & $(248 / 1322)$ & 15.4 & $(224 / 1455)$ & 13.1 & $(191 / 1458)$ & 15.3 & $(219 / 1435)$ & 16.2 & $(237 / 1461)$ \\
\hline
\end{tabular}

MS = Male Urethral Swab; MU = Male Urine; FS = Female Endocervical Swab; FU = Female Urine; PVS = Patient-Collected Vaginal Swab; CVS = Clinician-Collected Vaginal Swab.

Table 1a: Prevalence of C. trachomatis by Clinical Site and Overall as Determined by Aptima CT Assay Results Using PreservCyt Solution Liquid Pap Specimens

\begin{tabular}{ccc}
\hline Site & \multicolumn{2}{c}{ \% (\#positive I \#tested) } \\
\hline $\mathbf{1}$ & 17.0 & $(17 / 100)$ \\
\hline $\mathbf{2}$ & 3.2 & $(4 / 124)$ \\
\hline $\mathbf{3}$ & 7.4 & $(35 / 475)$ \\
\hline $\mathbf{4}$ & 4.2 & $(12 / 287)$ \\
\hline $\mathbf{5}$ & 5.4 & $(16 / 297)$ \\
\hline $\mathbf{6}$ & 5.5 & $(20 / 364)$ \\
\hline All & 6.3 & $(104 / 1647)$ \\
\hline
\end{tabular}




\section{Positive and Negative Predictive Values for Hypothetical Prevalence Rates in North America}

The estimated positive and negative predictive values (PPV and NPV) for different hypothetical prevalence rates using the Aptima CT Assay are shown in Table 2. These calculations are based on hypothetical prevalence rates and the overall sensitivity and specificity estimated from the patient infected status for three multi-center clinical investigations. The overall sensitivity and specificity for CT were $96.7 \%$ and $96.8 \%$, respectively (Table 2). The actual PPV and NPV for clinician-collected endocervical, vaginal and male urethral swab, patient-collected vaginal swab, and male and female urine specimens are shown in Table 6 for each clinical site and overall. The actual PPV and NPV for PreservCyt Solution liquid Pap specimens are shown in Table 6a.

Table 2: Positive and Negative Predictive Values for Hypothetical Prevalence Rates

\begin{tabular}{ccccc}
$\begin{array}{c}\text { Hypothetical } \\
\text { Prevalence Rate (\%) }\end{array}$ & Sensitivity (\%) & Specificity (\%) & PPV (\%) & NPV (\%) \\
\hline 1 & 96.7 & 96.8 & 23.5 & 100.0 \\
\hline 2 & 96.7 & 96.8 & 38.3 & 99.9 \\
\hline 5 & 96.7 & 96.8 & 61.6 & 99.8 \\
\hline 10 & 96.7 & 96.8 & 77.2 & 99.6 \\
\hline 15 & 96.7 & 94.3 & 99.4 \\
\hline 20 & 96.7 & 96.8 & 99.2 \\
\hline 25 & 96.7 & 96.8 & 91.0 & 98.9 \\
\hline 30 & 96.7 & 96.8 & 92.9 & 98.6
\end{tabular}

\section{Aptima CT Assay RLU Distribution}

Figure 3 shows the RLU distribution for the Aptima CT Assay for all specimen types in the clinical study except PreservCyt Solution liquid Pap specimens. Table 3 summarizes the RLU distribution for the total positive and total negative results, as well as the false positive and false negative results for each specimen type except PreservCyt Solution liquid Pap specimens relative to infected patient status. Across certain specimen types, there is a trend toward an increasing proportion of true positives as the RLU values increase. 


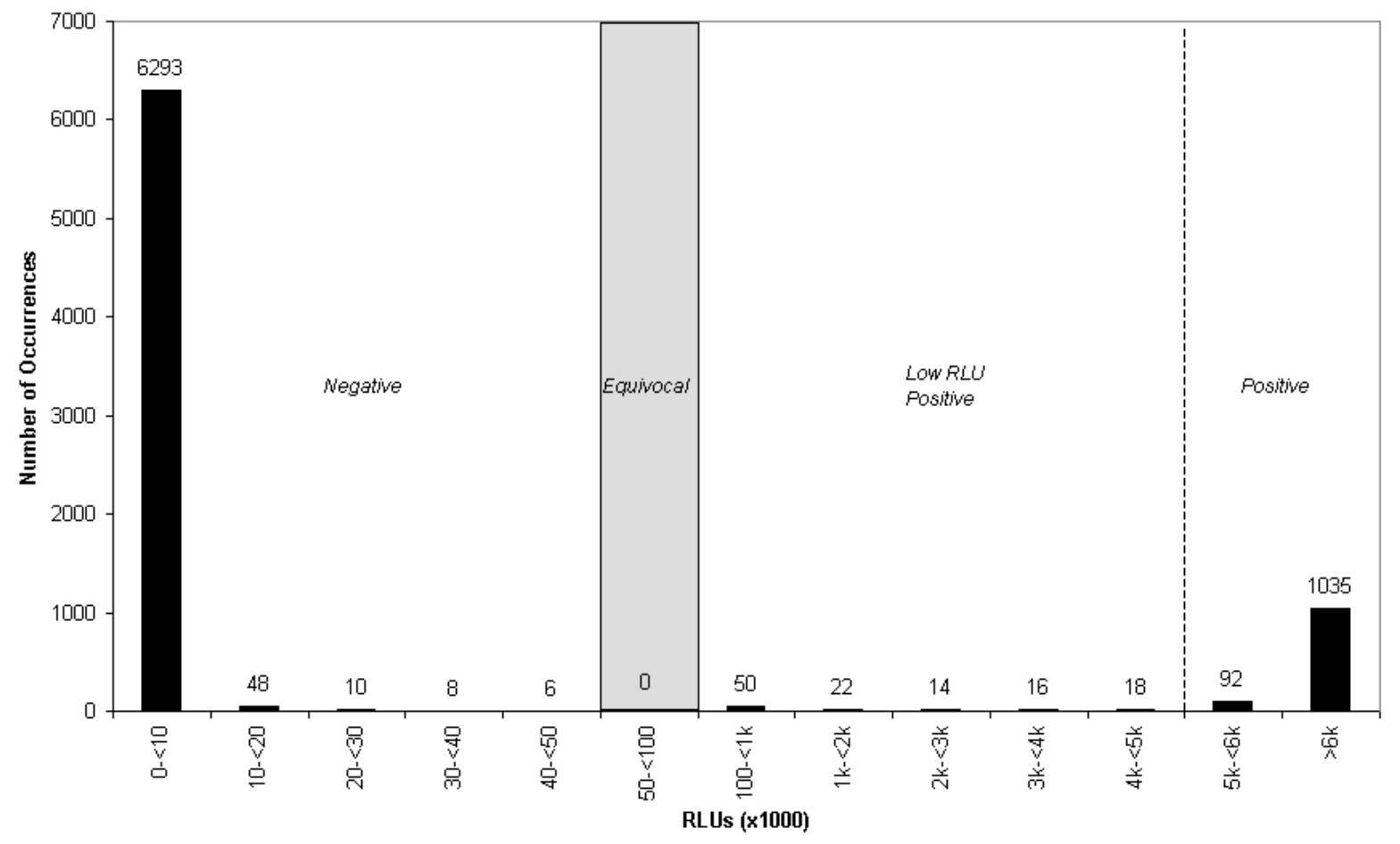

Figure 3. Frequency of RLU Distribution for the Aptima CT Assay 
Table 3: Aptima CT Assay RLU Distribution

\begin{tabular}{|c|c|c|c|c|c|c|c|c|c|c|c|c|c|}
\hline & \multicolumn{13}{|c|}{ RLUs (x 1000) } \\
\hline & $0<10$ & $10<20$ & $20<30$ & $30<40$ & $40<50$ & $50<100$ & $\begin{array}{l}100< \\
1000\end{array}$ & $\begin{array}{c}1000< \\
2000\end{array}$ & $\begin{array}{c}2000< \\
3000\end{array}$ & $\begin{array}{c}3000< \\
4000\end{array}$ & $\begin{array}{c}4000< \\
5000\end{array}$ & $\begin{array}{c}5000< \\
6000\end{array}$ & $>6000$ \\
\hline $\begin{array}{c}\text { Total } \\
\text { Positives }\end{array}$ & & & & & & 0 & 50 & 22 & 14 & 16 & 18 & 92 & 1035 \\
\hline $\begin{array}{c}\text { Total False } \\
\text { Positives }\end{array}$ & & & & & & 0 & 43 & 17 & 7 & 11 & 10 & 25 & 126 \\
\hline CVS & & & & & & 0 & 18 & 4 & 1 & 4 & 4 & 6 & 28 \\
\hline PVS & & & & & & 0 & 7 & 5 & 2 & 1 & 2 & 2 & 6 \\
\hline FS & & & & & & 0 & 9 & 2 & 3 & 2 & 2 & 5 & 26 \\
\hline MS & & & & & & 0 & 3 & 4 & 0 & 1 & 0 & 3 & 32 \\
\hline FU & & & & & & 0 & 5 & 2 & 0 & 1 & 0 & 6 & 12 \\
\hline MU & & & & & & 0 & 1 & 0 & 1 & 2 & 2 & 3 & 22 \\
\hline $\begin{array}{c}\text { Total } \\
\text { Negatives }\end{array}$ & 6293 & 48 & 10 & 8 & 6 & 0 & & & & & & & \\
\hline $\begin{array}{l}\text { Total False } \\
\text { Negatives }\end{array}$ & 31 & 1 & 0 & 1 & 0 & 0 & & & & & & & \\
\hline CVS & 4 & 0 & 0 & 1 & 0 & 0 & & & & & & & \\
\hline PVS & 1 & 0 & 0 & 0 & 0 & 0 & & & & & & & \\
\hline FS & 3 & 0 & 0 & 0 & 0 & 0 & & & & & & & \\
\hline MS & 4 & 1 & 0 & 0 & 0 & 0 & & & & & & & \\
\hline FU & 10 & 0 & 0 & 0 & 0 & 0 & & & & & & & \\
\hline MU & 9 & 0 & 0 & 0 & 0 & 0 & & & & & & & \\
\hline
\end{tabular}

CVS = Clinician-Collected Vaginal Swab; PVS = Asymptomatic Patient-Collected Vaginal Swab; FS = Female Endocervical Swab; MS = Male Urethral Swab; FU = Female Urine; $\mathbf{M U}=$ Male Urine.

Shaded column denotes equivocal zone. 


\section{DTS Systems Clinical Performance}

See Tigris DTS System Clinical Specimen Agreement following the DTS Systems Analytical Performance section for Tigris DTS System-specific clinical performance.

\section{Clinical Specimen Study - Endocervical Swab, Male Urethral Swab, Vaginal Swab, and Urine Specimens}

Clinician-collected endocervical, vaginal and male urethral swab, patient-collected vaginal swab, and male and female urine specimens were collected from 2,787 symptomatic and asymptomatic, male and female subjects attending OB/GYN, sexually transmitted disease (STD), teen, and family planning clinics at eight geographically diverse clinical sites in North America. Subjects were classified as symptomatic if symptoms such as discharge, dysuria, and pelvic pain were reported by the subject. Subjects were classified as asymptomatic if the subject did not report symptoms. Of the 1,392 asymptomatic subjects enrolled in the study, 2 were less than 16 years of age, 237 were between the ages of 16 and 20, 423 were between the ages of 21 and 25, and 730 were greater than 25 years of age. Of the 1,395 symptomatic subjects enrolled in the study, 211 were between the ages of 16 and 20, 494 were between the ages of 21 and 25, and 690 were greater than 25 years of age.

Three specimens were collected from each of the 1,322 eligible male subjects. Five specimens were collected from each of the 1,465 eligible female subjects. For male subjects, two randomized urethral swabs were collected followed by one urine specimen. For female subjects, one urine specimen was collected followed by one patient-collected vaginal swab, one clinician-collected vaginal swab, and two randomized endocervical swabs. Aptima CT Assay and Aptima Combo 2 Assay CT results were generated from the two vaginal swabs, one endocervical swab, one male urethral swab, and a male and female urine aliquot. The remaining endocervical swab, male urethral swab, and a male and female urine aliquot were tested using another commercially-available NAAT. Endocervical and male urethral swab specimens and male and female urine specimens tested in the Aptima Combo 2 Assay and the other commercially available NAAT were used as the reference NAATs to determine infected status for each subject. Specimen testing was conducted either at the site of subject enrollment or at an external testing site.

All performance calculations were based on the total number of Aptima CT Assay results for endocervical, vaginal and male urethral swab, and male and female urine specimens compared to a patient infected status algorithm for each gender. In the algorithm, the designation of a subject as being infected or not infected with CT was based on endocervical swab and urine specimen results from the commercially-available Aptima Combo 2 Assay and the other commercially-available NAAT. Subjects were considered infected with CT if two of the four endocervical swab and urine specimens tested positive in the Aptima Combo 2 Assay and the other reference NAAT (one specimen testing positive in each NAAT). Subjects were considered non-infected if less than two reference NAAT results were positive.

A total of 8,406 Aptima CT Assay results were used to calculate sensitivity and specificity. Sensitivity and specificity for CT by gender, specimen type and symptom status are presented in Table 4. Table 6 shows the Aptima CT Assay sensitivity, specificity, and predictive values compared to patient infected status for each clinical site and overall. Tables 7a-7d summarize the number of results from symptomatic and asymptomatic subjects designated as infected or non-infected with CT according to the patient infected status algorithm. 
Of the 2,787 subjects enrolled, there were 13 subjects with unknown CT patient infected status. Subjects were designated with an unknown patient infected status if results were missing that prevented conclusive determination of infected status. These subjects' results were not included in any performance calculations. Of the 8,452 Aptima CT Assay results from the multi-center clinical study, there was a small percentage $(8,0.09 \%)$ of specimens that initially tested invalid for CT. Upon repeat testing, there were no equivocal or invalid results.

Table 4: Sensitivity and Specificity of the Aptima CT Assay Relative to Patient Infected Status by Symptom Status and Overall

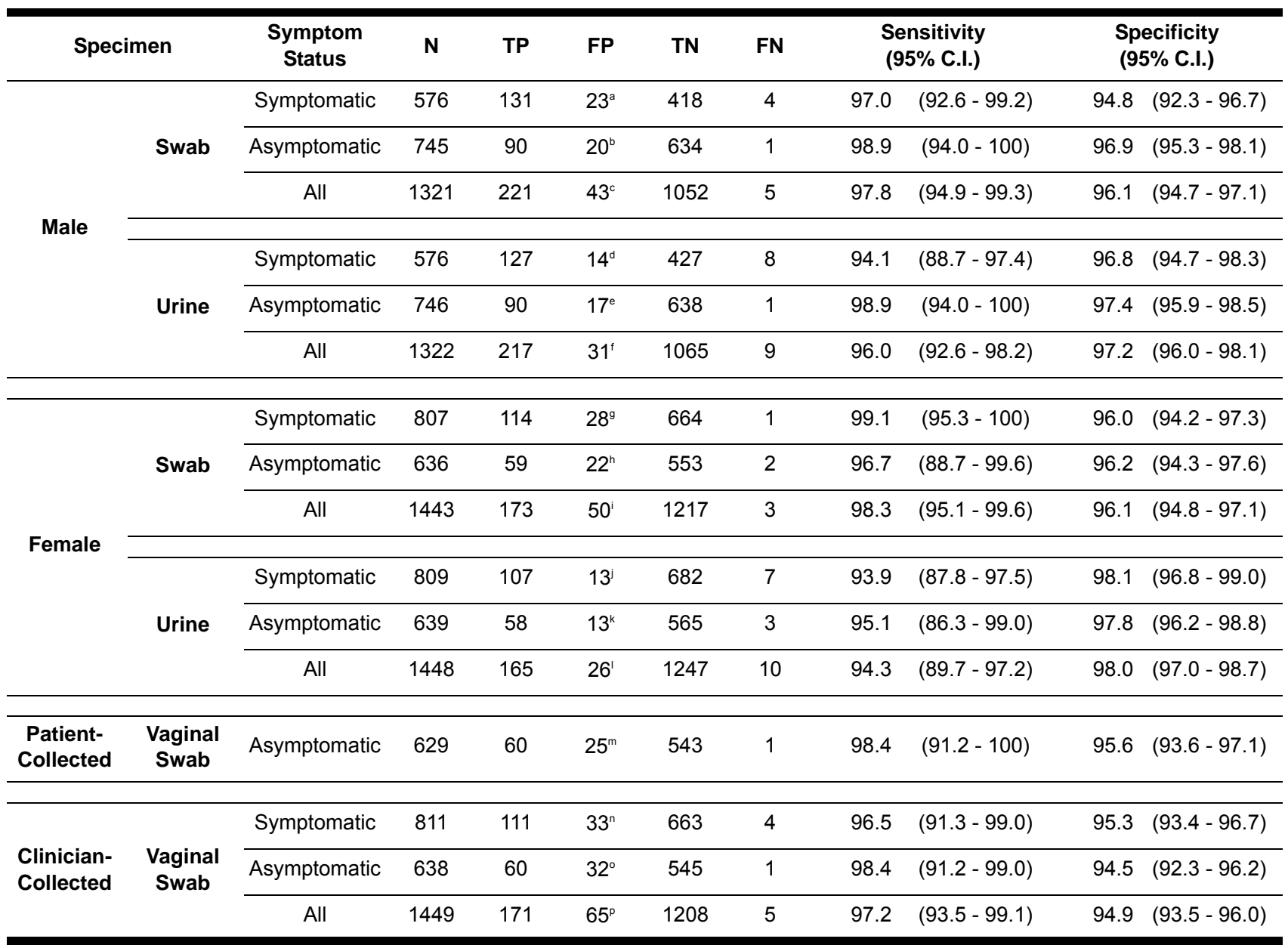

TP = True Positive; FP = False Positive $; \mathbf{T N}=$ True Negative; FN = False Negative .

Aptima Combo 2 Assay CT results: \# positive results / \# specimens tested a: 9/23; b: 14/20; c: 23/43; d: 6/14; e: 6/17; f: 12/31; g: 14/28; h:11/22; i: 25/50; j: 7/13;k: 5/13; l: 12/26; m: 15/25; n: 17/33; o: 15/32; p: 32/65. 


\section{Clinical Specimen Study - PreservCyt Solution Liquid Pap}

A prospective multi-center clinical study was conducted to evaluate the use of the PreservCyt Solution (a component of the ThinPrep 2000 System) as an alternative medium for gynecological specimens for the detection of CT by the Aptima CT Assay. One thousand six hundred forty-seven $(1,647)$ symptomatic and asymptomatic female subjects attending OB/ GYN, family planning, public health, women's, and STD clinics were evaluated in the clinical study. Of the 1,647 evaluable subjects, 1,288 were asymptomatic subjects and 359 were symptomatic subjects. Subjects were enrolled from sites with CT prevalence that ranged from $2.8 \%$ to $14.0 \%$.

Two specimens were collected from each eligible subject: one PreservCyt Solution liquid Pap specimen and one endocervical swab specimen. PreservCyt Solution liquid Pap specimens were collected with the spatula/cyto-brush or a broom-like brush cervical sampling device. The distribution of cervical sampling devices is summarized in Table 5 by specimen collection site and overall.

PreservCyt Solution liquid Pap specimens were processed in accordance with the ThinPrep 2000 Processor Operator's Manual and Aptima Specimen Transfer Kit Package Insert. After processing the PreservCyt Solution liquid Pap specimen with the ThinPrep 2000 Processor, the specimen was transferred into the Aptima Specimen Transfer Kit for testing with the Aptima CT Assay.

Sensitivity and specificity of the Aptima CT Assay in PreservCyt Solution liquid Pap specimens were calculated by comparing results to a patient infected status algorithm. The algorithm included Aptima Combo 2 Assay and Aptima CT Assay results in endocervical swab specimens. Both reference NAATs were required to be positive to establish an infected patient status. At least one reference NAAT was required to be negative to establish a noninfected patient status. Table 7e summarizes the frequency of test outcomes for the two reference NAATs.

Table 5 a shows the sensitivities and specificities of the Aptima CT Assay by symptom status and overall. Overall sensitivity was $95.6 \%$ (86/90). In symptomatic and asymptomatic subjects, sensitivities were $96.7 \%(29 / 30)$ and $95.0 \%$ (57/60), respectively. Overall specificity was $98.8 \%$ (1539/1557). In symptomatic and asymptomatic subjects, specificities were 98.8\% (325/329) and 98.9\% (1214/1228), respectively.

Table 6a shows the sensitivities and specificities of the Aptima CT Assay by specimen collection site and overall. Sensitivities ranged from $92.9 \%$ to $100 \%$. Specificities ranged from $96.5 \%$ to $100 \%$.

Table 5: Distribution of Cervical Sampling Device Used for PreservCyt Solution Liquid Pap Specimens

\begin{tabular}{cccccccc}
\hline \multirow{2}{*}{ Cervical Sampling Device Used } & \multicolumn{9}{c}{ Clinical Collection Site } & \multicolumn{2}{c}{ Total } \\
\cline { 2 - 8 } & $\mathbf{1}$ & $\mathbf{2}$ & $\mathbf{3}$ & $\mathbf{4}$ & $\mathbf{5}$ & $\mathbf{6}$ & 364 \\
\hline Spatula/Cytobrush & 0 & 124 & 475 & 287 & 57 & 1307 \\
\hline Broom-Type Device & 100 & 0 & 0 & 0 & 240 & 0 & 340 \\
\hline
\end{tabular}


Table 5a: Sensitivity and Specificity of the Aptima CT Assay Relative to Patient Infected Status by Symptom Status and Overall for PreservCyt Solution Liquid Pap Specimens

\begin{tabular}{|c|c|c|c|c|c|c|c|}
\hline Specimen & $\begin{array}{c}\text { Aptima CT } \\
\text { PreservCyt Solution } \\
\text { result }\end{array}$ & $+1+$ & $+l-$ & $-1+$ & $-1-$ & $\begin{array}{c}\text { Sensitivity (\%) } \\
(95 \% \mathrm{Cl})\end{array}$ & $\begin{array}{c}\text { Specificity (\%) } \\
(95 \% \mathrm{Cl})\end{array}$ \\
\hline \multirow{3}{*}{ Symptomatic } & Positive & 29 & 0 & 1 & 3 & \multirow{3}{*}{$\begin{array}{c}96.7(29 / 30) \\
(82.8-99.9)\end{array}$} & \multirow{3}{*}{$\begin{array}{c}98.8(325 / 329) \\
(96.9-99.7)\end{array}$} \\
\hline & Negative & 1 & 3 & 3 & 319 & & \\
\hline & Total & 30 & 3 & 4 & 322 & & \\
\hline \multirow{3}{*}{ Asymptomatic } & Positive & 57 & 0 & 1 & 13 & \multirow{3}{*}{$\begin{array}{l}95.0(57 / 60) \\
(86.1-99.0)\end{array}$} & \multirow{3}{*}{$\begin{array}{c}98.9(1214 / 1228) \\
(98.1-99.4)\end{array}$} \\
\hline & Negative & 3 & 2 & 11 & 1201 & & \\
\hline & Total & 60 & 2 & 12 & 1214 & & \\
\hline \multirow{3}{*}{ All } & Positive & 86 & 0 & 2 & 16 & \multirow{3}{*}{$\begin{array}{c}95.6(86 / 90) \\
(89.0-98.8)\end{array}$} & \multirow{3}{*}{$\begin{array}{c}98.8(1539 / 1557) \\
(98.2-99.3)\end{array}$} \\
\hline & Negative & 4 & 5 & 14 & 1520 & & \\
\hline & Total & 90 & 5 & 16 & 1536 & & \\
\hline
\end{tabular}

$+l+=$ Positive endocervical swab specimen result in the Aptima Combo 2 Assay/Positive endocervical swab specimen result in the Aptima CT Assay.

$+/$ - = Positive endocervical swab specimen result in the Aptima Combo 2 Assay/Negative endocervical swab specimen result in the Aptima CT Assay.

$-I_{+}=$Negative endocervical swab specimen result in the Aptima Combo 2 Assay/Positive endocervical swab specimen result in the Aptima CT Assay.

$-I-=$ Negative endocervical swab specimen result in the Aptima Combo 2 Assay/Negative endocervical swab specimen result in the Aptima CT Assay. 
Table 6: Sensitivity, Specificity and Predictive Values of the Aptima CT Assay Relative to Patient Infected Status by Clinical Site and Overall

\begin{tabular}{|c|c|c|c|c|c|c|c|c|c|c|c|c|c|c|}
\hline \multicolumn{2}{|c|}{ Specimen } & \multirow{2}{*}{$\begin{array}{c}\text { Site } \\
1\end{array}$} & \multirow{2}{*}{$\begin{array}{c}\mathbf{N} \\
252\end{array}$} & \multirow{2}{*}{$\begin{array}{l}\text { TP } \\
54\end{array}$} & \multirow{2}{*}{$\begin{array}{r}\text { FP } \\
14\end{array}$} & \multirow{2}{*}{$\begin{array}{c}\text { TN } \\
183\end{array}$} & \multirow{2}{*}{$\begin{array}{c}\text { FN } \\
1\end{array}$} & \multirow{2}{*}{$\begin{array}{c}\text { Prev. } \\
\text { (\%) }\end{array}$} & \multicolumn{2}{|c|}{ Sensitivity (95\% C.I.) } & \multicolumn{2}{|c|}{ Specificity (95\% C.I.) } & \multirow{2}{*}{$\begin{array}{c}\text { PPV } \\
\text { (\%) }\end{array}$} & \multirow{2}{*}{$\begin{array}{r}\text { NPV } \\
\text { (\%) }\end{array}$} \\
\hline & & & & & & & & & 98.2 & $(90.3-100)$ & 92.9 & $(88.4-96.1)$ & & \\
\hline & & 2 & 354 & 83 & 15 & 252 & 4 & 24.6 & 95.4 & $(88.6-98.7)$ & 94.4 & $(90.9-96.8)$ & 84.7 & 98.4 \\
\hline & & 3 & 4 & 1 & 0 & 3 & 0 & 25.0 & 100 & $(2.5-100)$ & 100 & $(29.2-100)$ & 100 & 100 \\
\hline & & 4 & N/A & N/A & $\mathrm{N} / \mathrm{A}$ & N/A & N/A & $\mathrm{N} / \mathrm{A}$ & $\mathrm{N} / \mathrm{A}$ & N/A & N/A & N/A & N/A & $\mathrm{N} / \mathrm{A}$ \\
\hline & Swab & 5 & 200 & 12 & 4 & 184 & 0 & 6.0 & 100 & $(73.5-100)$ & 97.9 & $(94.6-99.4)$ & 75.0 & 100 \\
\hline & & 6 & 304 & 59 & 10 & 235 & 0 & 19.4 & 100 & $(93.9-100)$ & 95.9 & $(92.6-98.0)$ & 85.5 & 100 \\
\hline & & 7 & 207 & 12 & 0 & 195 & 0 & 5.8 & 100 & $(73.5-100)$ & 100 & $(98.1-100)$ & 100 & 100 \\
\hline & & 8 & $\mathrm{~N} / \mathrm{A}$ & $\mathrm{N} / \mathrm{A}$ & $\mathrm{N} / \mathrm{A}$ & N/A & N/A & $\mathrm{N} / \mathrm{A}$ & $\mathrm{N} / \mathrm{A}$ & $\mathrm{N} / \mathrm{A}$ & N/A & N/A & N/A & N/A \\
\hline & & All & 1321 & 221 & 43 & 1052 & 5 & 17.1 & 97.8 & $(94.9-99.3)$ & 96.1 & $(94.7-97.1)$ & 83.7 & 99.4 \\
\hline \multicolumn{15}{|l|}{ Male } \\
\hline & \multirow{9}{*}{ Urine } & 1 & 252 & 54 & 9 & 188 & 1 & 21.8 & 98.2 & $(90.3-100)$ & 95.4 & $(91.5-97.9)$ & 85.7 & 99.5 \\
\hline & & 2 & 354 & 85 & 9 & 258 & 2 & 24.6 & 97.7 & $(91.9-99.7)$ & 96.6 & $(93.7-98.4)$ & 90.4 & 99.2 \\
\hline & & 3 & 4 & 1 & 0 & 3 & 0 & 25.0 & 100 & $(2.5-100)$ & 100 & $(29.2-100)$ & 100 & 100 \\
\hline & & 4 & $\mathrm{~N} / \mathrm{A}$ & N/A & $\mathrm{N} / \mathrm{A}$ & N/A & N/A & $N / A$ & $N / A$ & N/A & N/A & N/A & $\mathrm{N} / \mathrm{A}$ & N/A \\
\hline & & 5 & 200 & 12 & 4 & 184 & 0 & 6.0 & 100 & $(73.5-100)$ & 97.9 & $(94.6-99.4)$ & 75.0 & 100 \\
\hline & & 6 & 305 & 53 & 8 & 238 & 6 & 19.3 & 89.8 & $(79.2-96.2)$ & 96.7 & $(93.7-98.6)$ & 86.9 & 97.5 \\
\hline & & 7 & 207 & 12 & 1 & 194 & 0 & 5.8 & 100 & $(73.5-100)$ & 99.5 & $(97.2-100)$ & 92.3 & 100 \\
\hline & & 8 & N/A & N/A & $N / A$ & N/A & N/A & $\mathrm{N} / \mathrm{A}$ & $\mathrm{N} / \mathrm{A}$ & $N / A$ & N/A & N/A & N/A & $\mathrm{N} / \mathrm{A}$ \\
\hline & & All & 1322 & 217 & 31 & 1065 & 9 & 17.1 & 96.0 & $(92.6-98.2)$ & 97.2 & $(96.0-98.1)$ & 87.5 & 99.2 \\
\hline \multirow{19}{*}{ Female } & \multirow{9}{*}{ Swab } & 1 & 228 & 36 & 2 & 190 & 0 & 15.8 & 100 & $(90.3-100)$ & 99.0 & $(96.3-99.9)$ & 94.7 & 100 \\
\hline & & 2 & 198 & 52 & 18 & 128 & 0 & 26.3 & 100 & $(93.2-100)$ & 87.7 & $(81.2-92.5)$ & 74.3 & 100 \\
\hline & & 3 & 114 & 9 & 4 & 101 & 0 & 7.9 & 100 & $(66.4-100)$ & 96.2 & $(90.5-99.0)$ & 69.2 & 100 \\
\hline & & 4 & 260 & 19 & 11 & 229 & 1 & 7.7 & 95.0 & $(75.1-99.9)$ & 95.4 & $(91.9-97.7)$ & 63.3 & 99.6 \\
\hline & & 5 & 199 & 13 & 5 & 181 & 0 & 6.5 & 100 & $(75.3-100)$ & 97.3 & $(93.8-99.1)$ & 72.2 & 100 \\
\hline & & 6 & 294 & 33 & 9 & 252 & 0 & 11.2 & 100 & $(89.4-100)$ & 96.6 & $(93.6-98.4)$ & 78.6 & 100 \\
\hline & & 7 & 102 & 8 & 0 & 92 & 2 & 9.8 & 80.0 & $(44.4-97.5)$ & 100 & $(96.1-100)$ & 100 & 97.9 \\
\hline & & 8 & 48 & 3 & 1 & 44 & 0 & 6.3 & 100 & $(29.2-100)$ & 97.8 & $(88.2$ - 99.9) & 75.0 & 100 \\
\hline & & All & 1443 & 173 & 50 & 1217 & 3 & 12.2 & 98.3 & $(95.1-99.6)$ & 96.1 & $(94.8-97.1)$ & 77.6 & 99.8 \\
\hline & & & & & & & & & & & & & & \\
\hline & \multirow{9}{*}{ Urine } & 1 & 227 & 34 & 5 & 187 & 1 & 15.4 & 97.1 & $(85.1$ - 99.9) & 97.4 & $(94.0-99.1)$ & 87.2 & 99.5 \\
\hline & & 2 & 198 & 51 & 2 & 144 & 1 & 26.3 & 98.1 & $(89.7-100)$ & 98.6 & $(95.1-99.8)$ & 96.2 & 99.3 \\
\hline & & 3 & 113 & 9 & 1 & 103 & 0 & 8.0 & 100 & $(66.4-100)$ & 99.0 & $(94.8-100)$ & 90.0 & 100 \\
\hline & & 4 & 265 & 18 & 4 & 241 & 2 & 7.5 & 90.0 & $(68.3-98.8)$ & 98.4 & $(95.9-99.6)$ & 81.8 & 99.2 \\
\hline & & 5 & 199 & 11 & 4 & 182 & 2 & 6.5 & 84.6 & $(54.6-98.1)$ & 97.8 & $(94.6-99.4)$ & 73.3 & 98.9 \\
\hline & & 6 & 295 & 29 & 10 & 252 & 4 & 11.2 & 87.9 & $(71.8-96.6)$ & 96.2 & $(93.1-98.2)$ & 74.4 & 98.4 \\
\hline & & 7 & 102 & 10 & 0 & 92 & 0 & 9.8 & 100 & $(69.2-100)$ & 100 & $(96.1-100)$ & 100 & 100 \\
\hline & & 8 & 49 & 3 & 0 & 46 & 0 & 6.1 & 100 & $(29.2-100)$ & 100 & $(92.3-100)$ & 100 & 100 \\
\hline & & All & 1448 & 165 & 26 & 1247 & 10 & 12.1 & 94.3 & $(89.7-97.2)$ & 98.0 & $(97.0-98.7)$ & 86.4 & 99.2 \\
\hline
\end{tabular}


Table 6: Sensitivity, Specificity and Predictive Values of the Aptima CT Assay Relative to Patient Infected Status by Clinical Site and Overall (continued)

\begin{tabular}{|c|c|c|c|c|c|c|c|c|c|c|c|c|c|}
\hline Specimen & \multirow{2}{*}{$\begin{array}{c}\text { Site } \\
1\end{array}$} & \multirow{2}{*}{$\begin{array}{l}\mathbf{N} \\
70\end{array}$} & \multirow{2}{*}{$\begin{array}{c}\text { TP } \\
14\end{array}$} & \multirow{2}{*}{$\begin{array}{c}\text { FP } \\
4\end{array}$} & \multirow{2}{*}{$\begin{array}{l}\text { TN } \\
52\end{array}$} & \multirow{2}{*}{$\begin{array}{c}\text { FN } \\
0\end{array}$} & \multirow{2}{*}{$\begin{array}{c}\text { Prev. } \\
\text { (\%) }\end{array}$} & \multicolumn{2}{|c|}{ Sensitivity ( $95 \%$ C.I.) } & \multicolumn{2}{|c|}{ Specificity ( $95 \%$ C.I.) } & \multirow{2}{*}{$\begin{array}{l}\text { PPV } \\
\text { (\%) }\end{array}$} & \multirow{2}{*}{$\begin{array}{l}\text { NPV } \\
\text { (\%) } \\
100\end{array}$} \\
\hline \multirow{9}{*}{$\begin{array}{l}\text { Patient- } \\
\text { Collected }\end{array}$} & & & & & & & & 100 & $(76.8-100)$ & 92.9 & $(82.7-98.0)$ & & \\
\hline & 2 & 46 & 13 & 4 & 29 & 0 & 28.3 & 100 & $(75.3-100)$ & 87.9 & $(71.8-96.6)$ & 76.5 & 100 \\
\hline & 3 & 45 & 4 & 2 & 39 & 0 & 8.9 & 100 & $(39.8-100)$ & 95.1 & $(83.5-99.4)$ & 66.7 & 100 \\
\hline & 4 & 152 & 6 & 3 & 142 & 1 & 4.6 & 85.7 & $(42.1-99.6)$ & 97.9 & $(94.1-99.6)$ & 66.7 & 99.3 \\
\hline & 5 & 130 & 7 & 3 & 120 & 0 & 5.4 & 100 & $(59.0-100)$ & 97.6 & (93.0 - 99.5) & 70.0 & 100 \\
\hline & 6 & 75 & 8 & 5 & 62 & 0 & 10.7 & 100 & $(63.1-100)$ & 92.5 & $(83.4-97.5)$ & 61.5 & 100 \\
\hline & 7 & 68 & 5 & 2 & 61 & 0 & 7.4 & 100 & $(47.8-100)$ & 96.8 & $(89.0-99.6)$ & 71.4 & 100 \\
\hline & 8 & 43 & 3 & 2 & 38 & 0 & 7.0 & 100 & $(29.2-100)$ & 95.0 & $(83.1-99.4)$ & 60.0 & 100 \\
\hline & All & 629 & 60 & 25 & 543 & 1 & 9.7 & 98.4 & $(91.2-100)$ & 95.6 & $(93.6-97.1)$ & 70.6 & 99.8 \\
\hline \multirow{9}{*}{$\begin{array}{l}\text { Clinician- } \\
\text { Collected }\end{array}$} & 1 & 228 & 36 & 8 & 184 & 0 & 15.8 & 100 & $(90.3-100)$ & 95.8 & $(92.0-98.2)$ & 81.8 & 100 \\
\hline & 2 & 198 & 50 & 16 & 130 & 2 & 26.3 & 96.2 & $(86.8-99.5)$ & 89.0 & $(82.8-93.6)$ & 75.8 & 98.5 \\
\hline & 3 & 113 & 9 & 4 & 100 & 0 & 8.0 & 100 & $(66.4-100)$ & 96.2 & $(90.4-98.9)$ & 69.2 & 100 \\
\hline & 4 & 263 & 18 & 14 & 229 & 2 & 7.6 & 90.0 & $(68.3-98.8)$ & 94.2 & $(90.5-96.8)$ & 56.3 & 99.1 \\
\hline & 5 & 199 & 13 & 7 & 179 & 0 & 6.5 & 100 & $(75.3-100)$ & 96.2 & $(92.4-98.5)$ & 65.0 & 100 \\
\hline & 6 & 296 & 33 & 15 & 248 & 0 & 11.1 & 100 & $(89.4-100)$ & 94.3 & $(90.8-96.8)$ & 68.8 & 100 \\
\hline & 7 & 102 & 9 & 0 & 92 & 1 & 9.8 & 90.0 & $(55.5-99.7)$ & 100 & $(96.1-100)$ & 100 & 98.9 \\
\hline & 8 & 50 & 3 & 1 & 46 & 0 & 6.0 & 100 & $(29.2-100)$ & 97.9 & $(88.7-99.9)$ & 75.0 & 100 \\
\hline & All & 1449 & 171 & 65 & 1208 & 5 & 12.1 & 97.2 & $(93.5-99.1)$ & 94.9 & $(93.5-96.0)$ & 72.5 & 99.6 \\
\hline
\end{tabular}

TP = True Positive; FP = False Positive $; \mathbf{T N}=$ True Negative; FN = False Negative. 
Table 6a: Sensitivity, Specificity and Predictive Values of the Aptima CT Assay Relative to Patient Infected Status by Clinical Site and Overall for PreservCyt Solution Liquid Pap Specimens

\begin{tabular}{|c|c|c|c|c|c|c|c|c|c|c|}
\hline Site & $\begin{array}{c}\text { Aptima CT } \\
\text { PreservCyt } \\
\text { Solution } \\
\text { Result }\end{array}$ & $+1+$ & $+1-$ & $-1+$ & $-I-$ & $\begin{array}{l}\text { Prev } \\
\text { (\%) }\end{array}$ & $\begin{array}{c}\text { Sensitivity (\%) } \\
\text { (95\% C.I.) }\end{array}$ & $\begin{array}{c}\text { Specificity (\%) } \\
\text { (95\% C.I.) }\end{array}$ & PPV (\%) & NPV (\%) \\
\hline \multirow{3}{*}{1} & Positive & 14 & 0 & 1 & 2 & \multirow{3}{*}{14.0} & \multirow{3}{*}{$\begin{array}{c}100(14 / 14) \\
(76.8-100)\end{array}$} & \multirow{3}{*}{$\begin{array}{c}96.5(83 / 86) \\
(90.1-99.3)\end{array}$} & \multirow{3}{*}{82.4} & \multirow{3}{*}{100} \\
\hline & Negative & 0 & 0 & 0 & 83 & & & & & \\
\hline & Total & 14 & 0 & 1 & 85 & & & & & \\
\hline \multirow{3}{*}{2} & Positive & 4 & 0 & 0 & 0 & \multirow{3}{*}{3.2} & \multirow{3}{*}{$\begin{array}{c}100(4 / 4) \\
(39.8-100)\end{array}$} & \multirow{3}{*}{$\begin{array}{c}100(120 / 120) \\
(97.0-100)\end{array}$} & \multirow{3}{*}{100} & \multirow{3}{*}{100} \\
\hline & Negative & 0 & 0 & 2 & 118 & & & & & \\
\hline & Total & 4 & 0 & 2 & 118 & & & & & \\
\hline \multirow{3}{*}{3} & Positive & 29 & 0 & 0 & 6 & \multirow{3}{*}{6.5} & \multirow{3}{*}{$\begin{array}{c}93.5(29 / 31) \\
(78.6-99.2)\end{array}$} & \multirow{3}{*}{$\begin{array}{c}98.6(438 / 444) \\
(97.1-99.5)\end{array}$} & \multirow{3}{*}{82.9} & \multirow{3}{*}{99.5} \\
\hline & Negative & 2 & 0 & 2 & 436 & & & & & \\
\hline & Total & 31 & 0 & 2 & 442 & & & & & \\
\hline \multirow{3}{*}{4} & Positive & 8 & 0 & 0 & 4 & \multirow{3}{*}{2.8} & \multirow{3}{*}{$\begin{array}{c}100(8 / 8) \\
(63.1-100)\end{array}$} & \multirow{3}{*}{$\begin{array}{c}98.6(275 / 279) \\
(96.4-99.6)\end{array}$} & \multirow{3}{*}{66.7} & \multirow{3}{*}{100} \\
\hline & Negative & 0 & 3 & 1 & 271 & & & & & \\
\hline & Total & 8 & 3 & 1 & 275 & & & & & \\
\hline \multirow{3}{*}{5} & Positive & 13 & 0 & 0 & 3 & \multirow{3}{*}{4.7} & \multirow{3}{*}{$\begin{array}{c}92.9(13 / 14) \\
(66.1-99.8)\end{array}$} & \multirow{3}{*}{$\begin{array}{c}98.9(280 / 283) \\
(96.9-99.8)\end{array}$} & \multirow{3}{*}{81.3} & \multirow{3}{*}{99.6} \\
\hline & Negative & 1 & 1 & 4 & 275 & & & & & \\
\hline & Total & 14 & 1 & 4 & 278 & & & & & \\
\hline \multirow{3}{*}{6} & Positive & 18 & 0 & 1 & 1 & \multirow{3}{*}{5.2} & \multirow{3}{*}{$\begin{array}{c}94.7(18 / 19) \\
(74.0-99.9)\end{array}$} & & & \\
\hline & Negative & 1 & 1 & 5 & 337 & & & $\begin{array}{c}99.4(343 / 345) \\
(97.9-99.9)\end{array}$ & 90.0 & 99.7 \\
\hline & Total & 19 & 1 & 6 & 338 & & & & & \\
\hline & Positive & 86 & 0 & 2 & 16 & & & & & \\
\hline All & Negative & 4 & 5 & 14 & 1520 & 5.5 & $\begin{array}{l}95.6(86 / 90) \\
(89.0-98.8)\end{array}$ & $\begin{array}{c}98.8(1539 / 1557) \\
(98.2-99.3)\end{array}$ & 82.7 & 99.7 \\
\hline & Total & 90 & 5 & 16 & 1536 & & & & & \\
\hline
\end{tabular}

$+/+=$ Positive endocervical swab specimen result in the Aptima Combo 2 Assay/Positive endocervical swab specimen result in the Aptima CT Assay.

$+l-=$ Positive endocervical swab specimen result in the Aptima Combo 2 Assay/Negative endocervical swab specimen result in the Aptima CT Assay.

$-I_{+}=$Negative endocervical swab specimen result in the Aptima Combo 2 Assay/Positive endocervical swab specimen result in the Aptima CT Assay.

$-I-=$ Negative endocervical swab specimen result in the Aptima Combo 2 Assay/Negative endocervical swab specimen result in the Aptima CT Assay. 
Table 7a: Male Urethral Swab and Urine Results from Subjects Infected or Non-Infected with C. trachomatis According to Patient Infected Status

\begin{tabular}{|c|c|c|c|c|c|c|c|c|c|}
\hline \multirow[t]{2}{*}{ Patient Infected Status } & \multicolumn{2}{|c|}{$\begin{array}{c}\text { NAAT } 1 \\
\text { (Aptima Combo } 2 \\
\text { Assay) }\end{array}$} & \multicolumn{2}{|c|}{ NAAT 2} & \multicolumn{2}{|c|}{ Aptima CT Assay } & \multicolumn{2}{|c|}{ Symptom Status } & \multirow[t]{2}{*}{ Total } \\
\hline & MS & MU & MS & MU & MS & MU & Sympt. & Asympt. & \\
\hline Infected & + & + & + & + & + & + & 96 & 68 & 164 \\
\hline Infected & + & + & + & + & + & - & 5 & 1 & 6 \\
\hline Infected & + & + & + & - & + & + & 11 & 7 & 18 \\
\hline Infected & + & + & - & + & + & + & 13 & 11 & 24 \\
\hline Infected & + & + & - & + & + & - & 1 & 0 & 1 \\
\hline Infected & + & + & - & + & - & + & 1 & 0 & 1 \\
\hline Infected & + & - & + & + & + & + & 2 & 0 & 2 \\
\hline Infected & + & - & + & + & + & - & 1 & 0 & 1 \\
\hline Infected & + & - & + & - & + & - & 1 & 0 & 1 \\
\hline Infected & - & + & + & + & + & + & 1 & 0 & 1 \\
\hline Infected & - & + & - & + & + & + & 0 & 2 & 2 \\
\hline Infected & - & + & - & + & - & + & 3 & 1 & 4 \\
\hline Infected & - & + & $=$ & + & + & + & 0 & 1 & 1 \\
\hline Non-infected & + & + & - & - & + & + & 4 & 4 & 8 \\
\hline Non-infected & + & + & - & - & - & + & 1 & 0 & 1 \\
\hline Non-infected & + & - & - & - & + & + & 1 & 4 & 5 \\
\hline Non-infected & + & - & - & - & + & - & 4 & 6 & 10 \\
\hline Non-infected & + & - & - & - & - & + & 1 & 0 & 1 \\
\hline Non-infected & + & - & - & - & - & - & 3 & 0 & 3 \\
\hline Non-infected & - & + & - & - & + & + & 1 & 0 & 1 \\
\hline Non-infected & - & + & - & - & - & + & 0 & 2 & 2 \\
\hline Non-infected & - & + & - & - & - & - & 1 & 0 & 1 \\
\hline Non-infected & - & - & + & + & + & + & 1 & 0 & 1 \\
\hline Non-infected & - & - & - & + & - & - & 2 & 2 & 4 \\
\hline Non-infected & - & - & - & - & + & + & 1 & 1 & 2 \\
\hline Non-infected & - & - & - & - & + & - & 11 & 5 & 16 \\
\hline Non-infected & - & - & - & - & - & + & 4 & 4 & 8 \\
\hline Non-infected & - & - & - & - & - & - & 403 & 618 & 1021 \\
\hline Non-infected & - & - & - & N/A & - & + & 0 & 2 & 2 \\
\hline Non-infected & - & - & - & $\mathrm{N} / \mathrm{A}$ & - & - & 1 & 2 & 3 \\
\hline Non-infected & - & - & - & $=$ & - & - & 0 & 4 & 4 \\
\hline Non-infected & - & - & $=$ & - & - & - & 2 & 0 & 2 \\
\hline Non-infected & $\mathrm{N} / \mathrm{A}$ & - & - & - & $\mathrm{N} / \mathrm{A}$ & - & 0 & 1 & 1 \\
\hline Total & & & & & & & 576 & 746 & 1322 \\
\hline
\end{tabular}

N/A = Specimen not obtained or available for testing. The equal symbol (=) represents equivocal or indeterminate on repeat testing.

MS = Male Urethral Swab; MU = Male Urine . 
Table 7b: Female Endocervical Swab and Urine Results from Subjects Infected or Non-Infected with C. trachomatis According to Patient Infected Status

\begin{tabular}{|c|c|c|c|c|c|c|c|c|c|}
\hline \multirow[t]{2}{*}{ Patient Infected Status } & \multicolumn{2}{|c|}{$\begin{array}{c}\text { NAAT } 1 \\
\text { (Aptima Combo } 2 \\
\text { Assay) }\end{array}$} & \multicolumn{2}{|c|}{ NAAT 2} & \multicolumn{2}{|c|}{ Aptima CT Assay } & \multicolumn{2}{|c|}{ Symptom Status } & \multirow[t]{2}{*}{ Total } \\
\hline & FS & $\mathbf{F U}$ & FS & FU & FS & FU & Sympt. & Asympt. & \\
\hline Infected & + & + & + & + & + & + & 80 & 43 & 123 \\
\hline Infected & + & + & + & + & + & - & 1 & 1 & 2 \\
\hline Infected & + & + & + & $=$ & + & + & 1 & 0 & 1 \\
\hline Infected & + & + & - & + & + & + & 9 & 3 & 12 \\
\hline Infected & + & - & + & + & + & + & 3 & 1 & 4 \\
\hline Infected & + & - & + & + & + & - & 2 & 2 & 4 \\
\hline Infected & + & - & + & - & + & + & 2 & 0 & 2 \\
\hline Infected & + & - & + & - & + & N/A & 1 & 0 & 1 \\
\hline Infected & - & + & + & + & + & + & 0 & 1 & 1 \\
\hline Infected & - & + & - & + & + & + & 1 & 3 & 4 \\
\hline Infected & - & + & - & + & - & + & 1 & 2 & 3 \\
\hline Non-infected & + & + & - & - & + & + & 1 & 2 & 3 \\
\hline Non-infected & + & + & - & $\mathrm{N} / \mathrm{A}$ & + & + & 1 & 0 & 1 \\
\hline Non-infected & + & - & - & - & + & + & 0 & 2 & 2 \\
\hline Non-infected & + & - & - & - & + & - & 12 & 7 & 19 \\
\hline Non-infected & + & - & - & - & - & - & 0 & 1 & 1 \\
\hline Non-infected & - & + & - & - & + & + & 1 & 0 & 1 \\
\hline Non-infected & - & - & + & - & - & - & 1 & 1 & 2 \\
\hline Non-infected & - & - & - & + & - & - & 1 & 2 & 3 \\
\hline Non-infected & - & - & - & - & + & + & 0 & 2 & 2 \\
\hline Non-infected & - & - & - & - & + & - & 11 & 9 & 20 \\
\hline Non-infected & - & - & - & - & - & + & 5 & 4 & 9 \\
\hline Non-infected & - & - & - & - & - & - & 636 & 526 & 1162 \\
\hline Non-infected & - & - & - & - & - & $\mathrm{N} / \mathrm{A}$ & 1 & 0 & 1 \\
\hline Non-infected & - & - & - & $\mathrm{N} / \mathrm{A}$ & - & - & 2 & 3 & 5 \\
\hline Non-infected & - & - & - & $=$ & - & - & 12 & 10 & 22 \\
\hline Non-infected & - & - & $=$ & - & - & - & 1 & 1 & 2 \\
\hline Non-infected & - & N/A & - & - & - & $\mathrm{N} / \mathrm{A}$ & 1 & 1 & 2 \\
\hline Non-infected & N/A & - & - & - & N/A & - & 5 & 4 & 9 \\
\hline Non-infected & $=$ & - & - & - & + & + & 1 & 0 & 1 \\
\hline Non-infected & $=$ & - & - & - & + & - & 1 & 0 & 1 \\
\hline Total & & & & & & & 812 & 640 & 1452 \\
\hline
\end{tabular}

N/A = Specimen not obtained or available for testing. The equal symbol (=) represents equivocal or indeterminate on repeat testing.

FS = Female Endocervical Swab; FU = Female Urine. Sympt. $=$ Symptomatic Asympt. $=$ Asymptomatic . 
Table 7c: Asymptomatic Patient-Collected Vaginal Swab Results from Subjects Infected or Non-Infected with C. trachomatis According to Patient Infected Status

\begin{tabular}{|c|c|c|c|c|c|c|}
\hline \multirow[t]{2}{*}{ Patient Infected Status } & \multicolumn{2}{|c|}{$\begin{array}{c}\text { NAAT } 1 \\
\text { (Aptima Combo } 2 \text { Assay) }\end{array}$} & \multicolumn{2}{|c|}{ NAAT 2} & \multirow{2}{*}{$\begin{array}{c}\begin{array}{c}\text { Aptima CT } \\
\text { Assay }\end{array} \\
\text { PVS }\end{array}$} & \multirow[t]{2}{*}{ Total } \\
\hline & FS & FU & FS & FU & & \\
\hline Infected & + & + & + & + & + & 44 \\
\hline Infected & + & + & + & - & + & 5 \\
\hline Infected & + & + & - & + & + & 3 \\
\hline Infected & + & - & + & + & + & 3 \\
\hline Infected & - & + & + & + & + & 1 \\
\hline Infected & - & + & - & + & + & 4 \\
\hline Infected & - & + & - & + & - & 1 \\
\hline Non-infected & + & + & - & - & + & 2 \\
\hline Non-infected & + & - & - & - & + & 4 \\
\hline Non-infected & + & - & - & - & + & 1 \\
\hline Non-infected & + & - & - & - & - & 2 \\
\hline Non-infected & + & - & - & - & - & 3 \\
\hline Non-infected & - & + & - & - & + & 2 \\
\hline Non-infected & - & + & - & - & - & 2 \\
\hline Non-infected & - & - & + & - & - & 1 \\
\hline Non-infected & - & - & - & + & - & 2 \\
\hline Non-infected & - & - & - & - & + & 5 \\
\hline Non-infected & - & - & - & - & + & 10 \\
\hline Non-infected & - & - & - & - & - & 15 \\
\hline Non-infected & - & - & - & - & - & 500 \\
\hline Non-infected & - & - & - & - & - & 1 \\
\hline Non-infected & - & - & - & - & N/A & 1 \\
\hline Non-infected & - & - & - & - & N/A & 9 \\
\hline Non-infected & - & - & - & $\mathrm{N} / \mathrm{A}$ & - & 2 \\
\hline Non-infected & - & - & - & $\mathrm{N} / \mathrm{A}$ & $\mathrm{N} / \mathrm{A}$ & 1 \\
\hline Non-infected & - & - & - & $=$ & - & 1 \\
\hline Non-infected & - & - & - & $=$ & - & 8 \\
\hline Non-infected & - & - & - & $=$ & - & 1 \\
\hline Non-infected & - & - & $=$ & - & - & 1 \\
\hline Non-infected & - & $\mathrm{N} / \mathrm{A}$ & - & - & - & 1 \\
\hline Non-infected & $\mathrm{N} / \mathrm{A}$ & - & - & - & + & 1 \\
\hline Non-infected & $\mathrm{N} / \mathrm{A}$ & - & - & - & - & 3 \\
\hline Total & & & & & & 640 \\
\hline
\end{tabular}

N/A = Specimen not obtained or available for testing. The equal symbol (=) represents equivocal or indeterminate on repeat testing.

FS = Female Endocervical Swab; FU = Female Urine; CVS = Clinician-Collected Vaginal Swab; PVS = Asymptomatic Patient-Collected Vaginal Swab. 
Table 7d: Clinician-Collected Vaginal Swab Results from Subjects Infected or Non-Infected with C. trachomatis According to Patient Infected Status

\begin{tabular}{|c|c|c|c|c|c|c|c|c|}
\hline \multirow[t]{2}{*}{ Patient Infected Status } & \multicolumn{2}{|c|}{$\begin{array}{c}\text { NAAT } 1 \\
\text { (Aptima Combo } 2 \text { Assay) }\end{array}$} & \multicolumn{2}{|c|}{ NAAT 2} & \multirow{2}{*}{$\begin{array}{c}\text { Aptima CT } \\
\text { Assay } \\
\text { CVS }\end{array}$} & \multicolumn{2}{|c|}{ Symptom Status } & \multirow[t]{2}{*}{ Tota } \\
\hline & FS & FU & FS & FU & & Sympt. & Asympt. & \\
\hline Infected & + & + & + & + & + & 76 & 44 & 120 \\
\hline Infected & + & + & + & + & - & 2 & 0 & 2 \\
\hline Infected & + & + & + & + & + & 2 & 0 & 2 \\
\hline Infected & + & + & + & + & + & 1 & 0 & 1 \\
\hline Infected & + & + & + & - & + & 8 & 5 & 13 \\
\hline Infected & + & + & + & - & - & 1 & 0 & 1 \\
\hline Infected & + & + & + & - & + & 1 & 0 & 1 \\
\hline Infected & + & + & + & $=$ & + & 1 & 0 & 1 \\
\hline Infected & + & + & - & + & + & 9 & 3 & 12 \\
\hline Infected & + & - & + & + & + & 5 & 3 & 8 \\
\hline Infected & + & - & + & - & + & 7 & 0 & 7 \\
\hline Infected & - & + & + & + & + & 0 & 1 & 1 \\
\hline Infected & - & + & - & + & + & 1 & 4 & 5 \\
\hline Infected & - & + & - & + & - & 1 & 0 & 1 \\
\hline Infected & - & + & - & + & - & 0 & 1 & 1 \\
\hline Non-infected & + & + & - & - & + & 1 & 2 & 3 \\
\hline Non-infected & + & + & - & $\mathrm{N} / \mathrm{A}$ & + & 1 & 0 & 1 \\
\hline Non-infected & + & - & - & - & + & 3 & 4 & 7 \\
\hline Non-infected & + & - & - & - & - & 0 & 1 & 1 \\
\hline Non-infected & + & - & - & - & + & 2 & 2 & 4 \\
\hline Non-infected & + & - & - & - & - & 5 & 3 & 8 \\
\hline Non-infected & + & - & - & - & + & 1 & 0 & 1 \\
\hline Non-infected & + & - & - & - & - & 1 & 0 & 1 \\
\hline Non-infected & - & + & - & - & + & 5 & 2 & 7 \\
\hline Non-infected & - & + & - & - & - & 0 & 2 & 2 \\
\hline Non-infected & - & - & + & - & - & 1 & 1 & 2 \\
\hline Non-infected & - & - & - & + & - & 1 & 2 & 3 \\
\hline Non-infected & - & - & - & - & + & 4 & 5 & 9 \\
\hline Non-infected & - & - & - & - & - & 6 & 10 & 16 \\
\hline Non-infected & - & - & - & - & + & 16 & 15 & 31 \\
\hline Non-infected & - & - & - & - & - & 614 & 500 & 1114 \\
\hline Non-infected & - & - & - & - & N/A & 0 & 1 & 1 \\
\hline Non-infected & - & - & - & - & + & 0 & 1 & 1 \\
\hline Non-infected & - & - & - & - & - & 13 & 9 & 22 \\
\hline Non-infected & - & - & - & $\mathrm{N} / \mathrm{A}$ & - & 2 & 2 & 4 \\
\hline Non-infected & - & - & - & $\mathrm{N} / \mathrm{A}$ & - & 0 & 1 & 1 \\
\hline Non-infected & - & - & - & $=$ & + & 0 & 1 & 1 \\
\hline Non-infected & - & - & - & $=$ & - & 12 & 8 & 20 \\
\hline Non-infected & - & - & - & $=$ & N/A & 0 & 1 & 1 \\
\hline Non-infected & - & - & $=$ & - & - & 1 & 1 & 2 \\
\hline Non-infected & - & $\mathrm{N} / \mathrm{A}$ & - & - & - & 0 & 1 & 1 \\
\hline Non-infected & - & $\mathrm{N} / \mathrm{A}$ & - & - & $\mathrm{N} / \mathrm{A}$ & 1 & 0 & 1 \\
\hline Non-infected & $\mathrm{N} / \mathrm{A}$ & - & - & - & - & 0 & 1 & 1 \\
\hline Non-infected & $\mathrm{N} / \mathrm{A}$ & - & - & - & - & 5 & 3 & 8 \\
\hline
\end{tabular}


Table 7d: Clinician-Collected Vaginal Swab Results from Subjects Infected or Non-Infected with C. trachomatis According to Patient Infected Status (continued)

\begin{tabular}{|c|c|c|c|c|c|c|c|c|}
\hline \multirow{2}{*}{ Patient Infected Status } & \multicolumn{2}{|c|}{$\begin{array}{c}\text { NAAT } 1 \\
\text { (Aptima Combo } 2 \text { Assay) }\end{array}$} & \multicolumn{2}{|c|}{ NAAT 2} & \multirow{2}{*}{$\begin{array}{c}\text { Aptima CT } \\
\text { Assay }\end{array}$} & \multicolumn{2}{|c|}{ Symptom Status } & \multirow[t]{2}{*}{ Total } \\
\hline & FS & FU & FS & FU & & Sympt. & Asympt. & \\
\hline Non-infected & $=$ & - & - & - & - & 2 & 0 & 2 \\
\hline Total & & & & & & 812 & 640 & 1452 \\
\hline
\end{tabular}

N/A = Specimen not obtained or available for testing. The equal symbol (=) represents equivocal or indeterminate on repeat testing. FS = Female Endocervical Swab; FU = Female Urine; CVS = Clinician-Collected Vaginal Swab. Sympt. = Symptomatic; Asympt. = Asymptomatic.

Table 7e: PreservCyt Solution Liquid Pap Specimen Clinical Study Patient Infected Status Results for C. trachomatis

\begin{tabular}{cccccc}
\hline \multirow{2}{*}{ Patient Infected Status } & \multicolumn{2}{c}{ Endocervical Swab } & & \multicolumn{2}{c}{ Symptom Status } \\
\cline { 2 - 3 } \cline { 5 - 6 } & $\begin{array}{c}\text { Aptima Combo 2 } \\
\text { Assay }\end{array}$ & Aptima CT Assay & & Symptomatic & Asymptomatic \\
\hline Infected & Positive & Positive & & 30 & 60 \\
\hline Non-Infected & Negative & Negative & & 322 & 1214 \\
\hline Non-Infected & Negative & Positive & & 4 & 2 \\
\hline Non-Infected & Positive & Negative & & 3 & 1288 \\
\hline Total & & & 359 &
\end{tabular}

\section{RLU Distribution of Aptima Controls}

The distribution of the RLUs for the Aptima Positive Control, GC / Negative Control, CT and the Aptima Positive Control, CT / Negative Control, GC from all the Aptima CT Assay runs performed during the clinical specimen studies are presented in Table 8. 
Table 8: Distribution of RLU of the Aptima Controls During the Clinical Specimen Studies Including Endocervical, Vaginal and Male Urethral Swab, Male and Female Urine Specimens, and PreservCyt Liquid Pap Studies

\begin{tabular}{|c|c|c|c|}
\hline \multirow[b]{2}{*}{ Control } & \multirow[b]{2}{*}{ Statistics } & \multicolumn{2}{|c|}{ RLU (x1000) } \\
\hline & & $\begin{array}{c}\text { Swab and Urine } \\
\text { Specimen } \\
\text { Clinical Study }\end{array}$ & $\begin{array}{c}\text { PreservCyt Liquid } \\
\text { Pap Specimen } \\
\text { Clinical Study }\end{array}$ \\
\hline \multirow{8}{*}{ Positive Control, GC I Negative Control, CT } & $\mathrm{N}$ & 198 & 209 \\
\hline & Mean & 0.89 & 1.22 \\
\hline & SD & 2.94 & 2.63 \\
\hline & Maximum & 26 & 36 \\
\hline & $75^{\text {th }}$ Percentile & 1 & 1 \\
\hline & Median & 0 & 1 \\
\hline & $25^{\text {th }}$ Percentile & 0 & 1 \\
\hline & Minimum & 0 & 0 \\
\hline \multirow{8}{*}{ Positive Control, CT I Negative Control, GC } & $\mathrm{N}$ & 198 & 209 \\
\hline & Mean & 7007 & 6593 \\
\hline & SD & 776 & 709 \\
\hline & Maximum & 8884 & 10383 \\
\hline & $75^{\text {th }}$ Percentile & 7440 & 7025 \\
\hline & Median & 7066 & 6661 \\
\hline & $25^{\text {th }}$ Percentile & 6621 & 6205 \\
\hline & Minimum & 988 & 4419 \\
\hline
\end{tabular}

\section{Precision Study}

Aptima CT Assay precision (i.e., reproducibility) was evaluated at two external clinical sites and at Hologic. Aptima CT Assay precision was evaluated across three Aptima CT Assay kit lots, three study sites, six operators and 108 Aptima CT Assay runs. Two operators at each of the three testing sites performed a total of six Aptima CT Assay runs per kit lot for a total of 36 runs per kit lot. Each run was composed of a 12-member precision panel containing 0 to 2,000 fg/assay of CT rRNA. Reproducibility was established using spiked swab transport medium with rRNA. Reproducibility when testing swab and urine specimens containing target organism has not been determined. Table 9 presents the precision RLU data in terms of Mean, Standard Deviation, Coefficient of Variation (CV), and percent agreement with expected results for calculations of between-site, between-lot, between-operator, betweenrun, and within-run variability. 
Table 9: Aptima CT Assay Precision Data Using a 12-Member Precision Panel Containing 0 to 2,000 fg/ assay of CT rRNA

\begin{tabular}{|c|c|c|c|c|c|c|c|c|c|c|c|c|c|}
\hline \multirow[b]{2}{*}{ Concentration } & \multirow[b]{2}{*}{$\mathbf{N}$} & \multirow{2}{*}{$\begin{array}{c}\text { Mean } \\
\text { RLU } \\
(\times 1000)\end{array}$} & \multirow{2}{*}{$\begin{array}{c}\% \\
\text { Agrmt. }\end{array}$} & \multicolumn{2}{|c|}{ Within-Run } & \multicolumn{2}{|c|}{ Between-Site } & \multicolumn{2}{|c|}{ Between-Lot } & \multicolumn{2}{|c|}{ Between-Operator } & \multicolumn{2}{|c|}{ Between-Run } \\
\hline & & & & $\begin{array}{c}\text { SD } \\
(R L U \times 1000)\end{array}$ & $\begin{array}{l}\text { CV } \\
(\%)\end{array}$ & $\begin{array}{c}\text { SD } \\
(\mathrm{RLU} \times 1000)\end{array}$ & $\begin{array}{l}\text { CV } \\
\text { (\%) }\end{array}$ & $\begin{array}{c}\text { SD } \\
(\mathrm{RLU} \times 1000)\end{array}$ & $\begin{array}{l}\text { CV } \\
\text { (\%) }\end{array}$ & $\begin{array}{c}\text { SD } \\
(\mathrm{RLU} \times 1000)\end{array}$ & $\begin{array}{l}\text { CV } \\
(\%)\end{array}$ & $\begin{array}{c}\text { SD } \\
(\mathrm{RLU} \times 1000)\end{array}$ & $\begin{array}{l}\text { CV } \\
\text { (\%) }\end{array}$ \\
\hline $\operatorname{Neg}(0 \mathrm{fg} / \mathrm{mL})$ & 540 & 0.7 & 100 & 0.7 & N/A & 0.5 & $\mathrm{~N} / \mathrm{A}$ & 0.3 & N/A & 0.4 & N/A & 0 & $\mathrm{~N} / \mathrm{A}$ \\
\hline Low (12 fg/mL) & 216 & 7143.4 & 100 & 200.3 & 2.8 & 335.6 & 4.7 & 207.7 & 2.9 & 537.3 & 7.5 & 558.8 & 7.8 \\
\hline Mid $(250 \mathrm{fg} / \mathrm{mL})$ & 108 & 7084.9 & 100 & 162.2 & 2.3 & 275.1 & 3.9 & 159.5 & 2.3 & 546.3 & 7.7 & 578.2 & 8.2 \\
\hline $\operatorname{Mid}(2,500 \mathrm{fg} / \mathrm{mL})$ & 108 & 6991.1 & 100 & 150.7 & 2.2 & 279.4 & 4.0 & 117.8 & 1.7 & 532.3 & 7.6 & 534.9 & 7.7 \\
\hline $\begin{array}{c}\operatorname{High}(5,000-5,135 \\
f g / m L)\end{array}$ & 324 & 7133.4 & 100 & 229.2 & 3.2 & 301.0 & 4.2 & 129.0 & 1.8 & 531.7 & 7.5 & 618.3 & 8.7 \\
\hline
\end{tabular}

SD = Standard Deviation; CV(\%) = Percent Coefficient of Variation; \% Agrmt. = Percent Agreement.

Note: Variability from some factors may be numerically negative, which can occur if the variability due to those factors is very small. When this occurs, the variability as measured with SD and \%CV is set to zero (16). N/A = not applicable for negative analyte.

PreservCyt specimen within-laboratory precision with the Aptima CT Assay was determined by spiking PreservCyt vials with 20 CT IFU per vial (0.1 IFU per reaction) and $100 \mathrm{CT}$ IFU per vial (0.5 IFU per reaction). Vials containing 1,000 CT IFU per vial (5 IFU per reaction) and unspiked PreservCyt vials were tested as positive and negative controls. Ten vials spiked at each IFU level and ten unspiked vials were divided between two operators. The operators vortexed the vials and then transferred 14 aliquots $(1.0 \mathrm{~mL}$ each) per vial into 14 Aptima Transfer Tubes as per the Aptima Specimen Transfer Kit package insert. The operators were blinded to the samples' titers. Each of the resulting Pap-STM samples was tested once in the Aptima CT Assay. A total of five runs were performed over a five day period for 140 results at each IFU level. The results are summarized in Table 10.

Table 10: Aptima CT Assay Within-Laboratory Precision Data for PreservCyt using a 4-Member Precision Panel containing 0 to 1000 IFU/20 mL of CT cells

\begin{tabular}{|c|c|c|c|c|c|c|c|c|c|c|c|c|c|c|}
\hline \multirow{2}{*}{$\begin{array}{l}\text { Panel } \\
\text { Member }\end{array}$} & \multirow{2}{*}{$\begin{array}{l}\text { IFU/20mL } \\
\text { PreservCyt }\end{array}$} & \multirow{2}{*}{$\begin{array}{l}\text { IFUI } \\
\text { rxn }\end{array}$} & \multirow{2}{*}{$\mathbf{n}$} & \multirow{2}{*}{ Agreed } & \multirow{2}{*}{$\begin{array}{c}\% \\
\text { Agrmt. }\end{array}$} & \multirow{2}{*}{$\begin{array}{c}\text { Mean } \\
\text { RLU } \\
(\times 1000)\end{array}$} & \multicolumn{2}{|c|}{ Within-Operator } & \multicolumn{2}{|c|}{ Between-Day } & \multicolumn{2}{|c|}{$\begin{array}{l}\text { Between- } \\
\text { Operator }\end{array}$} & \multicolumn{2}{|c|}{ Total } \\
\hline & & & & & & & $\begin{array}{c}\text { SD } \\
(\times 1000)\end{array}$ & CV (\%) & $\begin{array}{c}\text { SD } \\
(\times 1000)\end{array}$ & CV (\%) & $\begin{array}{c}\text { SD } \\
(\times 1000)\end{array}$ & CV (\%) & $\begin{array}{c}\text { SD } \\
(\times 1000)\end{array}$ & CV (\%) \\
\hline A & 20 & 0.1 & 140 & 140 & 100 & 6501.7 & 734.8 & 11.3 & 0 & 0.0 & 546.9 & 8.4 & 916 & 14.1 \\
\hline B & 100 & 0.5 & 140 & $138^{*}$ & 98.6 & 6337.7 & 1054.7 & 16.6 & 0 & 0.0 & 947.2 & 14.9 & 1417.6 & 22.4 \\
\hline C & 1000 & 5 & 140 & 140 & 100 & 6521.9 & 909 & 13.9 & 247.1 & 3.8 & 393.9 & 6 & 1021 & 15.7 \\
\hline D & 0 & 0 & 140 & 140 & 100 & 1.2 & 0.8 & $\mathrm{~N} / \mathrm{A}$ & 0 & $\mathrm{~N} / \mathrm{A}$ & 0.4 & $\mathrm{~N} / \mathrm{A}$ & 0.9 & $\mathrm{~N} / \mathrm{A}$ \\
\hline
\end{tabular}

* discordant results were one negative result and 1 equivocal result

Note: Variability from some factors may be numerically negative, which can occur if the variability due to those factors is very small. When this occurs, the variability as measured with SD and \%CV is set to zero (16). N/A = not applicable for negative panel members. Operator $=$ Run. Samples with discordant results were included in the signal variability analysis. 


\section{DTS Systems Analytical Performance}

See Tigris DTS System Analytical Performance following the Tigris DTS System Clinical Specimen Agreement section for Tigris DTS System-specific analytical performance.

\section{Analytical Sensitivity}

C. trachomatis analytical sensitivity (limit of detection) was determined by directly comparing dilutions of CT organisms in cell culture and in the Aptima CT assay. The analytical sensitivity claim for the assay is one Inclusion-Forming Unit (IFU) per assay (7.25 IFU/swab, $5 \mathrm{IFU} / \mathrm{mL}$ urine, and $9.75 \mathrm{IFU} / \mathrm{mL}$ PreservCyt Solution liquid Pap) for all $15 \mathrm{CT}$ serovars (A, $\mathrm{B}, \mathrm{Ba}, \mathrm{C}, \mathrm{D}, \mathrm{E}, \mathrm{F}, \mathrm{G}, \mathrm{H}, \mathrm{I}, \mathrm{J}, \mathrm{K}, \mathrm{L} 1, \mathrm{~L} 2$ and L3). However, dilutions of less than one IFU/assay of all serovars tested positive.

\section{Analytical Specificity}

A total of 154 culture isolates were evaluated using the Aptima CT Assay. These isolates included 86 organisms that may be isolated from the urogenital tract and 68 additional organisms that represent a phylogenetic cross-section of organisms. The tested organisms included bacteria, fungi, yeast, parasites and viruses. All organisms except C. psittaci, C. pneumoniae, $U$. urealyticum and the viruses were tested at $1.0 \times 10^{6}$ cells/assay in KOVATrol/Urine Transport Media and 60 organisms were tested in Swab Transport Media. The Chlamydia and Neisseria organisms were tested in the PreservCyt Solution media. C. psittaci VR601 was tested at $8.0 \times 10^{4}$ cells/assay and C. psittaci VR125 was tested at $1.0 \times 10^{5}$ cells/assay. C. pneumoniae was tested at $4 \times 10^{3}$ cells/assay and $U$. urealyticum was tested at $6.7 \times 10^{6}$ cells/assay. The viruses were tested as follows: (a) herpes simplex virus I: $2.5 \times 10^{4} \mathrm{TCID}_{50}$ /assay, (b) herpes simplex virus II: $6.0 \times 10^{4} \mathrm{TCID}_{50}$ /assay, (c) human papillomavirus 16: $2.9 \times 10^{6}$ DNA copies/assay and (d) cytomegalovirus: $4.8 \times 10^{5}$ cells/assay. The list of organisms tested is shown in Table 11. 
Table 11: Analytical Specificity

\begin{tabular}{|c|c|c|}
\hline Organism & Organism & Organism \\
\hline Achromobacter xerosis & Escherichia coli & Neisseria mucosa (3) \\
\hline Acinetobacter calcoaceticus & Flavobacterium meningosepticum & Neisseria sicca (3) \\
\hline Acinetobacter Iwoffi & Fusobacterium nucleatum & Neisseria subflava (14) \\
\hline Actinomyces israelii & Gardnerella vaginalis & Neisseria perflava \\
\hline Actinomyces pyogenes & Gemella haemolysans & Neisseria polysaccharea \\
\hline Aerococcus viridans & Haemophilus ducreyi & Paracoccus denitrificans \\
\hline Aeromonas hydrophila & Haemophilus influenzae & Peptostreptococcus anaerobius \\
\hline Agrobacterium radiobacter & Herpes simplex virus I & Peptostreptococcus productus \\
\hline Alcaligenes faecalis & Herpes simplex virus II & Plesiomonas shigelloides \\
\hline Bacillus subtilis & Human papilloma virus 16 & Propionibacterium acnes \\
\hline Bacteriodes fragilis & Kingella dentrificans & Proteus mirabilis \\
\hline Bacteriodes ureolyticus & Kingella kingae & Proteus vulgaris \\
\hline Bifidobacterium adolescentis & Klebsiella oxytoca & Providencia stuartii \\
\hline Bifidobacterium brevi & Klebsiella pneumoniae & Pseudomonas aeruginosa \\
\hline Branhamella catarrhalis & Lactobacillus acidophilus & Pseudomonas fluorescens \\
\hline Brevibacterium linens & Lactobacillus brevis & Pseudomonas putida \\
\hline Campylobacter jejuni & Lactobacillus jensonii & Rahnella aquatilis \\
\hline Candida albicans & Lactobacillus lactis & Rhodospirillum rubrum \\
\hline Candida glabrata & Legionella pneumophila (2) & Saccharomyces cerevisiae \\
\hline Candida parapsilosis & Leuconostoc paramensenteroides & Salmonella minnesota \\
\hline Candida tropicalis & Listeria monocytogenes & Salmonella typhimurium \\
\hline Chlamydia pneumoniae & Micrococcus luteus & Serratia marcescens \\
\hline Chlamydia psittaci (2) & Moraxella lacunata & Staphylococcus saprophyticus \\
\hline Chromobacterium violaceum & Moraxella osloensis & Staphylococcus aureus \\
\hline Citrobacter freundii & Morganella morganii & Staphylococcus epidermidis \\
\hline Clostridium perfringens & Mycobacterium smegmatis & Streptococcus agalactiae \\
\hline Corynebacterium genitalium & Mycoplasma genitalium & Streptococcus bovis \\
\hline Corynebacterium xerosis & Mycoplasma hominis & Streptococcus mitis \\
\hline Cryptococcus neoformans & N. meningitidis Serogroup A & Streptococcus mutans \\
\hline Cytomegalovirus & N. meningitidis Serogroup B & Streptococcus pneumoniae \\
\hline Deinococcus radiodurans & N. meningitidis Serogroup C (4) & Streptococcus pyogenes \\
\hline Derxia gummosa & N. meningitidis Serogroup D & Streptococcus salivarius \\
\hline Eikenella corrodens & N. meningitidis Serogroup $Y$ & Streptococcus sanguis \\
\hline Enterobacter aerogenes & N. meningitidis Serogroup W135 & Streptomyces griseinus \\
\hline Enterobacter cloacae & Neisseria cinerea (4) & Trichomonas vaginalis \\
\hline Entercoccus avium & Neisseria dentrificans & Ureaplasma urealyticum \\
\hline Entercoccus faecalis & Neisseria elongata (3) & Vibrio parahaemolyticus \\
\hline Entercoccus faecium & Neisseria flava & Yersinia enterocolitica \\
\hline Erwinia herbicola & Neisseria flavescens (2) & \\
\hline Erysipelothrix rhusiopathiae & Neisseria lactamica (9) & \\
\hline
\end{tabular}

$(n)=$ number of strains tested. All organisms tested produced a negative result in the Aptima CT Assay. 


\section{Interfering Substances}

The following interfering substances were individually spiked into swab, PreservCyt Solution liquid Pap and/or urine specimens: $10 \%$ blood, contraceptive jelly, spermicide, moisturizer, hemorrhoidal anesthetic, body oil, powder, anti-fungal cream, vaginal lubricants, feminine spray and leukocytes $\left(1 \times 10^{6}\right.$ cells $\left./ \mathrm{mL}\right)$. The following interfering substances were individually spiked into urine specimens: $30 \%$ blood, urine analytes, protein, glucose, ketones, bilirubin, nitrate, urobilinogen, $\mathrm{pH} 4$ (acidic), $\mathrm{pH} 9$ (alkaline), leukocytes $\left(1 \times 10^{6} \mathrm{cells} / \mathrm{mL}\right)$, cellular debris, vitamins, minerals, acetaminophen, aspirin and ibuprofen. All were tested for potential assay interference in the absence and presence of CT at the estimated rRNA equivalent of 1 cell/assay ( $5 \mathrm{fg} /$ assay). The rRNA equivalents were calculated based on the genome size and estimated DNA:RNA ratio/cell of each organism. No interference was observed with any of the tested substances. No inhibitors of amplification were observed in the Aptima CT Assay.

\section{Recovery}

Escherichia coli, Gardnerella vaginalis, Lactobacillus acidophilus, Bacteroides ureolyticus, and Staphylococcus epidermidis ( $1 \times 10^{8}$ cells/assay) were added to samples containing the rRNA equivalent of approximately one CT IFU $(5 \mathrm{fg})$. These additions did not interfere with the amplification and detection of CT rRNA using the Aptima CT Assay.

\section{Specimen Stability Studies}

\section{A. Swab and Urine Specimens}

Data to support the recommended shipping and storage conditions for endocervical, urethral and vaginal swab samples were generated with pooled negative swab samples. Pooled samples were spiked with CT at a final concentration of 1 IFU per reaction. The spiked samples were held at $-70^{\circ} \mathrm{C},-20^{\circ} \mathrm{C}, 4^{\circ} \mathrm{C}$, and $30^{\circ} \mathrm{C}$. Samples were tested in duplicate at days $0,20,77$, and 117 . All test conditions were positive for CT at all times and temperatures.

Data to support the recommended shipping and storage conditions for urine samples were generated with female and male negative urine samples. The urine samples were spiked with CT at a final concentration of 10 IFU per reaction. Two sets of the spiked urine samples were held at $30^{\circ} \mathrm{C}$ for 24 hours prior to being added to the Urine Transport Media (UTM). The two sets of UTM samples then were held at $4{ }^{\circ} \mathrm{C}$ and $30^{\circ} \mathrm{C}$, and tested in triplicate at days $0,1,5,20$, and 35. All samples were positive for CT at all timepoints. The two sets of UTM samples were also tested after 116 days of storage at $-20^{\circ} \mathrm{C}$ and $-70^{\circ} \mathrm{C}$. All samples were positive for CT under both storage conditions.

\section{B. PreservCyt Solution Liquid Pap Specimens}

Data to support the recommended shipping and storage conditions for PreservCyt Solution liquid Pap samples were generated with negative processed and unprocessed liquid Pap samples. For the unprocessed samples, four pools of PreservCyt Solution samples were tested after being stored in the PreservCyt Solution vial. Each specimen pool was spiked with 1 to $10 \mathrm{IFU} \mathrm{CT/assay,} \mathrm{held} \mathrm{at} 2^{\circ} \mathrm{C}, 10^{\circ} \mathrm{C}$, and $30^{\circ} \mathrm{C}$, then tested at baseline and on days $5,7,8,14,18,21,25$ and 36 . All of the spiked samples were positive for CT at all times and temperatures.

For the processed samples, four pools of PreservCyt Solution samples were used to determine processed specimen stability at $2^{\circ} \mathrm{C}$ to $30^{\circ} \mathrm{C}$. Each negative sample pool was spiked with 1 to $10 \mathrm{IFU}$ CT/assay, then tested at baseline. Prior to processing, the PreservCyt Solution samples were stored at $30^{\circ} \mathrm{C}$ for seven (7) days to simulate the time 
lapse between sample collection, Pap processing and shipment to a microbiology testing lab. After seven days at $30^{\circ} \mathrm{C}, 1 \mathrm{~mL}$ aliquots of each pool were transferred to an Aptima Specimen Transfer Tube and tested at baseline before being placed at $2^{\circ} \mathrm{C}, 10^{\circ} \mathrm{C}$, and $30^{\circ} \mathrm{C}$. The processed samples were then tested for 17 days stored at $30^{\circ} \mathrm{C}$ and 36 days stored at $2^{\circ} \mathrm{C}$ to $10^{\circ} \mathrm{C}$. All of the spiked samples were positive for $\mathrm{CT}$ at all times and temperatures.

Data to support longer storage conditions were generated from four pools of negative processed PreservCyt Solution samples tested at below freezing temperatures. Each pool was spiked with 1 to 10 IFU CT/assay, then tested at baseline. Each pool was first placed at $30^{\circ} \mathrm{C}$ for 14 days and then stored at $-20^{\circ} \mathrm{C}$ or $-70^{\circ} \mathrm{C}$ over the course of 106 days. All of the spiked samples were positive for $\mathrm{CT}$ at all times and temperatures.

C. Additional Frozen (at $-20^{\circ} \mathrm{C}$ ) Specimen Stability Study

Data to support the recommended storage condition at $-20^{\circ} \mathrm{C}$ for endocervical swab, urethral swab, vaginal swab, female urine, male urine, and PreservCyt Solution liquid Pap specimens were generated using 90 specimens for each type with negative result, where 30 specimens were spiked with CT at 1.0 IFU per reaction; 30 specimens were spiked at 0.1 IFU per reaction; and 30 specimens were unspiked. The specimens were stored at $-20^{\circ} \mathrm{C}$ and were tested at days 0,200 , and 400 days. All spiked specimens met the acceptance criteria of $95 \%$ agreement with expected results. 


\section{Tigris DTS System Clinical Specimen Agreement}

\section{Tigris DTS System Agreement}

Agreement between Aptima CT Assay results generated on the fully automated Tigris DTS System and semi-automated DTS Systems was evaluated by testing endocervical swab, male urethral swab, male and female urine, vaginal swab, and PreservCyt liquid Pap specimens. Each of the clinical specimens was tested individually with the Aptima CT Assay on both the Tigris DTS System and DTS Systems at Hologic. The order of testing was not randomized. Specimens identified for inclusion were tested on the Tigris DTS System followed by testing on DTS Systems.

\section{Clinical Specimen Agreement Study-Endocervical Swab, Male Urethral Swab, Female and Male Urine, Vaginal Swab, and PreservCyt Liquid Pap Specimens}

Female and male subjects attending STD, family planning, and OB/GYN clinics from eight geographically diverse sites with low to high prevalence for CT contributed endocervical swab, male urethral swab, female and male urine, vaginal swab, and PreservCyt liquid Pap specimens. The specimens were transferred directly to Hologic for testing while the PreservCyt liquid Pap specimens were processed at 2 cytopathology laboratories before being transferred. At Hologic, endocervical swab, male urethral swab, female and male urine specimens were first screened with Aptima Combo 2 Assay on the Tigris DTS System, and the vaginal swab and PreservCyt liquid Pap specimens were screened with Aptima Combo 2 Assay on the DTS Systems. Specimens with final invalid or equivocal results were not selected in the Aptima CT Clinical Specimen Agreement Study.

Two hundred and five female swabs (87 endocervical and 118 vaginal), 120 male urethral swab, 98 female urine, 115 male urine, and 116 PreservCyt liquid Pap specimens with Aptima Combo 2 Assay CT positive and negative results were selected for comparison testing between the Tigris DTS System and the DTS Systems for the Aptima CT Assay. Specimens with initial invalid or equivocal results were retested using the same system on which the result was generated. One female urine specimen had an initial equivocal result on the DTS Systems; when retested, the final result was valid. One male urine specimen had an initial invalid result on the Tigris DTS System; when retested, the final result was valid. One female urine specimen had an initial equivocal result on the Tigris DTS System; this specimen was retested, however, the specimen had expired, so the final result was equivocal.

Table 12 shows the positive, negative, and overall agreements for all paired results for each specimen type by symptomatic status. Specimens are relatively imbalanced by symptomatic and asymptomatic status but overall agreements for symptomatic subjects were $98.5 \%$ (131/ 133 ) for female swabs (combined endocervical and vaginal swabs), $100 \%(60 / 60)$ for male urethral swab, $98.2 \%(55 / 56)$ for female urine specimens, $100 \%(60 / 60)$ for male urine specimens, and $100 \%$ (81/81) for PreservCyt liquid Pap specimens. For asymptomatic subjects, overall agreements were $100 \%$ for 72 female swabs, 60 male urethral swabs, 42 female urine, 55 male urine specimens, and 35 PreservCyt liquid Pap specimens, respectively. For 'All' (symptomatic and asymptomatic combined) subjects, overall agreement was $99.0 \%$ (203/205) for female swab (combined endocervical and vaginal swabs), $100 \%$ $(120 / 120)$ for male urethral swab, $99.0 \%(97 / 98)$ for female urine, $100 \%(115 / 115)$ for male urine, and $100 \%$ (116/116) for PreservCyt liquid Pap specimens. Due to the relatively smaller specimen number from asymptomatic subjects, these findings may not be generalizable to Aptima CT-Tigris System testing with specimens from asymptomatic subjects. 
Refer to Tables 4 and 5a for Aptima CT Assay sensitivity and specificity estimates from testing on the DTS Systems. Sensitivity and specificity of the Aptima CT Assay when using the Tigris DTS System would be expected to be similar given the agreement findings.

Table 12: Clinical Specimen Agreement Study: Positive, Negative, and Overall Agreements by Symptom Status

\begin{tabular}{|c|c|c|c|c|c|c|c|c|c|c|}
\hline Symptom & Specimen & Gender & $\mathbf{n}$ & $\begin{array}{c}\text { DTS+ } \\
\text { Tigris+ }\end{array}$ & $\begin{array}{l}\text { DTS+ } \\
\text { Tigris- }\end{array}$ & $\begin{array}{c}\text { DTS- } \\
\text { Tigris+ }\end{array}$ & $\begin{array}{l}\text { DTS- } \\
\text { Tigris- }\end{array}$ & $\begin{array}{c}\text { Positive \% } \\
\text { Agreement } \\
(95 \% \mathrm{Cl})\end{array}$ & $\begin{array}{c}\text { Negative \% } \\
\text { Agreement } \\
(95 \% \mathrm{CI})\end{array}$ & $\begin{array}{c}\text { Overall \% } \\
\text { Agreement } \\
(95 \% \mathrm{Cl})\end{array}$ \\
\hline \multirow{5}{*}{ Sympt. } & \multirow{2}{*}{ Swab } & Female* $^{*}$ & 133 & 63 & 1 & 1 & 68 & $\begin{array}{c}98.4 \\
(91.6-100)\end{array}$ & $\begin{array}{c}98.6 \\
(92.2-100)\end{array}$ & $\begin{array}{c}98.5 \\
(94.7-99.8)\end{array}$ \\
\hline & & Male & 60 & 42 & 0 & 0 & 18 & $\begin{array}{c}100 \\
(91.6-100)\end{array}$ & $\begin{array}{c}100 \\
(81.5-100)\end{array}$ & $\begin{array}{c}100 \\
(94.0-100)\end{array}$ \\
\hline & \multirow{2}{*}{ Urine } & Female & 56 & 33 & 0 & $1^{1}$ & 22 & $\begin{array}{c}100 \\
(89.4-100)\end{array}$ & $\begin{array}{c}95.7 \\
(78.1-99.9)\end{array}$ & $\begin{array}{c}98.2 \\
(90.4-100)\end{array}$ \\
\hline & & Male & 60 & 41 & 0 & 0 & 19 & $\begin{array}{c}100 \\
(91.4-100)\end{array}$ & $\begin{array}{c}100 \\
(82.4-100)\end{array}$ & $\begin{array}{c}100 \\
(94.0-100)\end{array}$ \\
\hline & PreservCyt & Female & 81 & 39 & 0 & 0 & 42 & $\begin{array}{c}100 \\
(91.0-100)\end{array}$ & $\begin{array}{c}100 \\
(91.6-100)\end{array}$ & $\begin{array}{c}100 \\
(95.5-100)\end{array}$ \\
\hline \multirow{5}{*}{ Asympt. } & \multirow{2}{*}{ Swab } & Female* $^{*}$ & 72 & 41 & 0 & 0 & 31 & $\begin{array}{c}100 \\
(91.4-100)\end{array}$ & $\begin{array}{c}100 \\
(88.8-100)\end{array}$ & $\begin{array}{c}100 \\
(95.0-100)\end{array}$ \\
\hline & & Male & 60 & 23 & 0 & 0 & 37 & $\begin{array}{c}100 \\
(85.2-100)\end{array}$ & $\begin{array}{c}100 \\
(90.5-100)\end{array}$ & $\begin{array}{c}100 \\
(94.0-100)\end{array}$ \\
\hline & \multirow{2}{*}{ Urine } & Female & 42 & 23 & 0 & 0 & 19 & $\begin{array}{c}100 \\
(85.2-100)\end{array}$ & $\begin{array}{c}100 \\
(82.4-100)\end{array}$ & $\begin{array}{c}100 \\
(91.6-100)\end{array}$ \\
\hline & & Male & 55 & 20 & 0 & 0 & 35 & $\begin{array}{c}100 \\
(83.2-100)\end{array}$ & $\begin{array}{c}100 \\
(90.0-100)\end{array}$ & $\begin{array}{c}100 \\
(93.5-100)\end{array}$ \\
\hline & PreservCyt & Female & 35 & 25 & 0 & 0 & 10 & $\begin{array}{c}100 \\
(86.3-100)\end{array}$ & $\begin{array}{c}100 \\
(69.2-100)\end{array}$ & $\begin{array}{c}100 \\
(90.0-100)\end{array}$ \\
\hline \multirow{5}{*}{ All } & \multirow{2}{*}{ Swab } & Female* $^{*}$ & 205 & 104 & 1 & 1 & 99 & $\begin{array}{c}99.0 \\
(94.8-100)\end{array}$ & $\begin{array}{c}99.0 \\
(94.6-100)\end{array}$ & $\begin{array}{c}99.0 \\
(96.5-99.9)\end{array}$ \\
\hline & & Male & 120 & 65 & 0 & 0 & 55 & $\begin{array}{c}100 \\
(94.5-100)\end{array}$ & $\begin{array}{c}100 \\
(93.5-100)\end{array}$ & $\begin{array}{c}100 \\
(97.0-100)\end{array}$ \\
\hline & \multirow{2}{*}{ Urine } & Female & 98 & 56 & 0 & $1^{1}$ & 41 & $\begin{array}{c}100 \\
(93.6-100)\end{array}$ & $\begin{array}{c}97.6 \\
(87.4-99.9)\end{array}$ & $\begin{array}{c}99.0 \\
(94.4-100)\end{array}$ \\
\hline & & Male & 115 & 61 & 0 & 0 & 54 & $\begin{array}{c}100 \\
(94.1-100)\end{array}$ & $\begin{array}{c}100 \\
(93.4-100)\end{array}$ & $\begin{array}{c}100 \\
(96.8-100)\end{array}$ \\
\hline & PreservCyt & Female & 116 & 64 & 0 & 0 & 52 & $\begin{array}{c}100 \\
(94.4-100)\end{array}$ & $\begin{array}{c}100 \\
(93.2-100)\end{array}$ & $\begin{array}{c}100 \\
(96.9-100)\end{array}$ \\
\hline
\end{tabular}

"+" denotes a positive result, "-" a negative result, $\mathrm{Cl}=$ confidence interval.

*Endocervical and Vaginal Swab samples combined.

${ }^{1}$ Specimen had a final equivocal result on the Tigris DTS System. 


\section{Precision Study}

The effect of several factors on the variability of Aptima CT Assay performance on the Tigris DTS System was evaluated using 12-member STD reproducibility panels. Panel members contained 0 to $5,000 \mathrm{fg}$ CT rRNA/assay. The panel included panel members with CT concentrations at the analytical sensitivity claim of $5 \mathrm{fg} \mathrm{CT}$ rRNA/assay.

The panels were tested at one external testing site and at Hologic using two Aptima CT Assay reagent lots. At Hologic, two operators each performed three valid worklists per reagent lot on each of two Tigris DTS System instruments. At the external testing site, two operators each performed three valid worklists per reagent lot on one Tigris DTS System instrument. One worklist consisted of run controls and six 12-member panels.

Reproducibility was determined by calculating the agreement between the final assay results and the expected outcome for each panel member. Reproducibility was also assessed by calculating the SD and coefficient of variation (CV) of signal with respect to sites, operators, lots, and worklists. CVs were not calculated for CT-negative panel members due to low signal values that could theoretically equal zero. Table 13 shows the reproducibility results. All Aptima CT Assay results on the Tigris DTS System agreed with the expected results. CV values were less than or equal to $3.4 \%$. These data indicate excellent reproducibility of the Aptima CT Assay using the Tigris DTS System.

Table 13: Tigris DTS System Precision Data

\begin{tabular}{|c|c|c|c|c|c|c|c|c|c|c|c|c|c|}
\hline \multirow{2}{*}{$\begin{array}{c}\text { Conc } \\
\text { (fg rRNA } \\
\text { per assay) }\end{array}$} & \multirow{2}{*}{$\mathbf{n}$} & \multirow{2}{*}{$\begin{array}{c}\text { Mean } \\
\text { RLU } \\
(\times 1000)\end{array}$} & \multirow{2}{*}{$\begin{array}{c}\% \\
\text { Agrmt }\end{array}$} & \multicolumn{2}{|c|}{ Between-Site } & \multicolumn{2}{|c|}{$\begin{array}{l}\text { Between- } \\
\text { Operator }\end{array}$} & \multicolumn{2}{|c|}{ Between-Lot } & \multicolumn{2}{|c|}{$\begin{array}{l}\text { Between- } \\
\text { Worklist }\end{array}$} & \multicolumn{2}{|c|}{ Within-Worklist } \\
\hline & & & & $\begin{array}{c}\text { SD }^{1} \\
(\times 1000)\end{array}$ & $\begin{array}{l}C^{1} \\
(\%)\end{array}$ & $\begin{array}{c}\text { SD } \\
(\times 1000)\end{array}$ & $\begin{array}{l}\text { CV } \\
\text { (\%) }\end{array}$ & $\begin{array}{c}S^{S D^{1}} \\
(\times 1000)\end{array}$ & $\begin{array}{l}\text { CV } \\
\text { (\%) }\end{array}$ & $\begin{array}{c}\text { SD } \\
(\times 1000)\end{array}$ & $\begin{array}{l}\text { CV } \\
\text { (\%) }\end{array}$ & $\begin{array}{c}\text { SD } \\
(\times 1000)\end{array}$ & $\begin{array}{l}\text { CV } \\
\text { (\%) }\end{array}$ \\
\hline 0 & 863 & 2.9 & 100 & 1.4 & N/A & 0.3 & $\mathrm{~N} / \mathrm{A}$ & 0.0 & $\mathrm{~N} / \mathrm{A}$ & 0.2 & $\mathrm{~N} / \mathrm{A}$ & 2.2 & $\mathrm{~N} / \mathrm{A}$ \\
\hline 5 & 432 & 7041 & 100 & 32.0 & 0.5 & 217 & 3.1 & 63.7 & 0.9 & 174 & 2.5 & 206 & 2.9 \\
\hline 50 & $433^{2}$ & 7090 & 100 & 0.0 & 0.0 & 224 & 3.2 & 93.1 & 1.3 & 168 & 2.4 & 189 & 2.7 \\
\hline 500 & $431^{3}$ & 7130 & 100 & 0.0 & 0.0 & 240 & 3.4 & 96.9 & 1.4 & 164 & 2.3 & 217 & 3.0 \\
\hline 5,000 & 432 & 7152 & 100 & 0.0 & 0.0 & 208 & 2.9 & 85.7 & 1.2 & 179 & 2.5 & 211 & 3.0 \\
\hline
\end{tabular}

Agrmt $=$ Agreement, Conc $=$ Concentration, $\mathrm{CV}=$ Coefficient of Variation, N/A $=$ Not Applicable for negative samples, $\mathrm{RLU}=$ Relative Light Units, SD = Standard Deviation.

${ }^{1} \mathrm{SD}$ and $\mathrm{CV}$ values are set to 0 and $0.0 \%$, respectively, according to the random effects model, if the variability due to this source relative to random errors and/or variation of other sources is numerically negative.

${ }^{2}$ One worklist included 1 additional replicate of a panel member with $50 \mathrm{fg} \mathrm{rRNA} / \mathrm{assay}$.

${ }^{3}$ One worklist was missing 1 replicate of a panel member with $500 \mathrm{fg}$ rRNA/assay. 


\section{Tigris DTS System Analytical Performance}

\section{Analytical Sensitivity Equivalence Study}

Sensitivity panels in endocervical swab pool, vaginal specimen pool, urine specimen pool, and PreservCyt liquid Pap specimen pool were prepared at CT rRNA equivalent of 1 IFU per assay (7.25 IFU/swab and $5 \mathrm{IFU} / \mathrm{mL}$ urine) and tested 60 replicates on the Tigris DTS System. Percent positivity (95\% C.I.) on the Tigris DTS System for endocervical swab specimen was $100 \%$ (95.1 - 100), for vaginal swab specimen was $100 \%$ (95.1 - 100), for urine specimen was 100\% (95.1 - 100), and for PreservCyt liquid Pap specimen was 100\% (95.1 - 100).

\section{CT rRNA Spiked Clinical Panel Study}

The CT rRNA spiked clinical panel study evaluated agreement between the two systems (Tigris DTS System and DTS Systems) using six Hologic prepared CT clinical panels spiked with 0 to $5,000 \mathrm{fg}$ rRNA/assay of CT. The CT clinical panels were created from endocervical swab, vaginal swab, urethral swab, male urine, female urine, and PreservCyt liquid Pap specimens that had negative Aptima CT results on the DTS Systems when tested at Hologic. The negative specimens were pooled by specimen type, spiked or not spiked with CT rRNA and aliquotted as replicates of each panel member. Replicates of each of 6-panel members with different spiked rRNA levels were combined to create one clinical panel for each specimen type. Each panel contained a total of 132 replicates.

Table 14 shows the percent agreement for each level of rRNA in the endocervical swab, vaginal swab, urethral swab, male urine, female urine, and PreservCyt liquid Pap panels, respectively, with expected CT results for the Tigris DTS System and for the DTS Systems. The concentration ranged from 1 log below to 3 logs above the $5 \mathrm{fg}$ rRNA/assay for CT. Also shown in Table 14 are the overall percent agreements of the clinical panel study between the Tigris DTS System and DTS Systems. 
Table 14: CT rRNA Spiked Clinical Panel Agreement Study

\begin{tabular}{|c|c|c|c|c|c|c|c|}
\hline \multicolumn{2}{|c|}{ Specimen } & \multirow{2}{*}{$\begin{array}{c}\text { Panel Member } \\
\text { No Target }\end{array}$} & \multirow{2}{*}{$\begin{array}{c}\begin{array}{c}\text { Concentration } \\
\text { (fg rRNA/Assay) }\end{array} \\
0\end{array}$} & \multirow{2}{*}{$\begin{array}{c}\text { Replicates } \\
12\end{array}$} & \multirow{2}{*}{$\begin{array}{c}\begin{array}{c}\text { Tigris \% } \\
\text { Agreement }\end{array} \\
100\end{array}$} & \multirow{2}{*}{$\begin{array}{c}\begin{array}{c}\text { DTS \% } \\
\text { Agreement }\end{array} \\
100\end{array}$} & \multirow{2}{*}{$\begin{array}{c}\text { Overall \% Agreement } \\
\text { between Tigris and } \\
\text { DTS }(95 \% \mathrm{Cl})\end{array}$} \\
\hline \multirow{15}{*}{ Swab } & \multirow{5}{*}{ Endocervical } & & & & & & \\
\hline & & Very Low & 0.5 & 30 & 100 & 100 & \multirow{4}{*}{$100(97.2-100)$} \\
\hline & & Low & 5 & 30 & 100 & 100 & \\
\hline & & Medium & 50 & 30 & 100 & 100 & \\
\hline & & High & 5,000 & 30 & 100 & 100 & \\
\hline & \multirow{5}{*}{ Vaginal } & No Target & 0 & 12 & 100 & 100 & \multirow{5}{*}{$100(97.2-100)$} \\
\hline & & Very Low & 0.5 & 30 & 100 & 100 & \\
\hline & & Low & 5 & 30 & 100 & 100 & \\
\hline & & Medium & 50 & 30 & 100 & 100 & \\
\hline & & High & 5,000 & 30 & 100 & 100 & \\
\hline & \multirow{5}{*}{ Urethral } & No Target & 0 & 12 & 100 & 100 & \multirow{5}{*}{$100(97.2-100)$} \\
\hline & & Very Low & 0.5 & 30 & 100 & 100 & \\
\hline & & Low & 5 & 30 & 100 & 100 & \\
\hline & & Medium & 50 & 30 & 100 & 100 & \\
\hline & & High & 5,000 & 30 & 100 & 100 & \\
\hline \multirow{10}{*}{ Urine } & \multirow{5}{*}{ Male } & No Target & 0 & 12 & $91.7(11 / 12)$ & 100 & \multirow{5}{*}{$99.2(95.9-100)$} \\
\hline & & Very Low & 0.5 & 30 & 100 & 100 & \\
\hline & & Low & 5 & 30 & 100 & 100 & \\
\hline & & Medium & 50 & 30 & 100 & 100 & \\
\hline & & High & 5,000 & 30 & 100 & 100 & \\
\hline & \multirow{5}{*}{ Female } & No Target & 0 & 12 & 100 & 100 & \multirow{5}{*}{$100(97.2-100)$} \\
\hline & & Very Low & 0.5 & 30 & 100 & 100 & \\
\hline & & Low & 5 & 30 & 100 & 100 & \\
\hline & & Medium & 50 & 30 & 100 & 100 & \\
\hline & & High & 5,000 & 30 & 100 & 100 & \\
\hline \multirow{5}{*}{\multicolumn{2}{|c|}{ PreservCyt liquid Pap }} & No Target & 0 & 12 & 100 & 100 & \multirow{5}{*}{$100(97.2-100)$} \\
\hline & & Very Low & 0.5 & 30 & 100 & 100 & \\
\hline & & Low & 5 & 30 & 100 & 100 & \\
\hline & & Medium & 50 & 30 & 100 & 100 & \\
\hline & & High & 5,000 & 30 & 100 & 100 & \\
\hline
\end{tabular}




\section{Analytical Specificity Equivalence Study}

For a nucleic acid amplification assay, analytical specificity with respect to individual organisms is largely determined by the chemistry of the assay (e.g. oligonucleotide sequences) rather than by the platform. Because the reagents for the Aptima CT Assay are identical between the Tigris DTS System and the DTS Systems, analytical specificity experiments on the Tigris DTS System were designed to focus on the most challenging culture isolates. These organisms included those known to cross-react in other amplification assays. Twenty-four (24) culture isolates were selected from the panel of organisms in Table 11, including 3 organisms that are most closely related to CT. All of the organisms tested produced negative results on the Tigris DTS System.

\section{Interfering Substances Equivalence Study}

Whole blood, a substance commonly found in urogenital specimens and known to interfere in some amplification assays, was used to establish that the Tigris DTS System tolerates similar levels of potentially interfering substances as does the DTS Systems. Fresh blood was added to clinical swab, vaginal swab, urine, and PreservCyt liquid Pap specimen pools, then tested for potential assay interference in the absence and presence of CT target at the estimated rRNA equivalent of one CT IFU/assay ( 5 fg/assay). The rRNA equivalents were calculated based on the genome size and estimated DNA:RNA ratio/cell of each organism. Specimens were tested on two Tigris DTS Systems. All samples containing target nucleic acid were positive when tested at a level of $10 \%$ blood in swab specimens, vaginal swab specimens, PreservCyt liquid Pap specimens, and 30\% blood in urine specimens. All samples that did not contain target were negative for CT. These results indicate that at the levels tested, whole blood is unlikely to affect the CT result on the Tigris DTS System.

\section{Carryover Studies for the Tigris DTS System}

To establish that the Tigris DTS System minimizes the risk of false positive results arising from carryover contamination, a study was conducted using spiked panels on three Tigris DTS Systems. The study used $20 \%$ high-target samples containing $1 \times 10^{6} \mathrm{fg} \mathrm{CT} \mathrm{rRNA} / \mathrm{mL}$, which were randomly spaced amongst $80 \%$ negative samples containing swab transport media. In the study, 576 high-target samples and 2,376 negative samples were tested across the three Tigris DTS Systems. Table 15 shows the overall carryover rate was averaged at $0.21 \%(5 / 2364)$. A total of 12 negative samples were reported as invalid and were excluded from the calculation. A separate analysis was conducted on a subset of the study population comprised of the negative samples that immediately followed a high-target positive. The carryover rate for this subset of the population was averaged at $0.47 \%(2 / 424)$. For false positives in this subset, the carryover rate ranged from $0 \%$ to $1.43 \%$ across the three Tigris DTS Systems. These results demonstrate that carryover contamination is minimized on the Tigris DTS System. 
Table 15: Summary of Overall Tigris DTS System Carryover

\begin{tabular}{ccccc}
\hline Instrument & $\begin{array}{c}\text { \# Valid } \\
\text { Negative Tests }\end{array}$ & $\begin{array}{c}\text { Total \# CT False } \\
\text { Positive Results }\end{array}$ & $\begin{array}{c}\text { \% CT False } \\
\text { Positive Results }\end{array}$ & $\begin{array}{c}\text { Confidence Intervals } \\
\mathbf{( 9 5 \% ~ C l ) ~}\end{array}$ \\
\hline Tigris 1 & 789 & $2^{\mathrm{a}}$ & 0.25 & $0.03-0.91$ \\
\hline Tigris 2 & 783 & $3^{\mathrm{b}}$ & 0.38 & $0.08-1.12$ \\
\hline Tigris 3 & 792 & $0^{\mathrm{c}}$ & 0.00 & $0.00-0.38$ \\
\hline All Instruments & 2364 & 5 & 0.21 & $0.07-0.49$ \\
\hline
\end{tabular}

a. Tigris 1 had no false CT positive result directly following a high-target positive.

b. Tigris 2 had two false CT positive results directly following a high-target positive.

c. Tigris 3 had no false CT positive result directly following a high-target positive. 


\section{Bibliography}

1. Beem, M. O., and E. M. Saxon. 1977. Respiratory tract colonization and a distinctive pneumonia syndrome in infants infected with Chlamydia trachomatis. NEJM 296:306-310.

2. Buimer, M., G. J. J. Van Doornum, S. Ching, P. G. H. Peerbooms, P. K. Plier, D. Ram, and H. H. Lee. 1996. Detection of Chlamydia trachomatis and Neisseria gonorrhoeae by Ligase chain reaction-based assays with clinical specimens from various sites: implications for diagnostic testing and screening. J. Clin. Microbiol. 34:2395-2400.

3. Cates, Jr., W., and J. N. Wasserheit. 1991. Genital chlamydia infections: epidemiology and reproductive sequelae. Am. J. Obstet. Gynecol. 164:1771-1781.

4. Centers for Disease Control and Prevention. 2002. Screening Tests to Detect Chlamydia trachomatis and Neisseria gonorrhoeae infections. United States Morbid. and Mortal. Weekly Rep. 51(RR-15)

5. Centers for Disease Control and Prevention. 2011. Sexually Transmitted Disease Surveillance 2010. Atlanta, GA: U.S. Department of Health and Human Services. November.

6. Chernesky, M. A., D. Jang, J. Sellors, K. Luinstra, S. Chong, S. Castriciano, and J. B. Mahony. 1996. Urinary inhibitors of polymerase chain reaction and Ligase chain reaction and testing of multiple specimens may contribute to lower assay sensitivities for diagnosing Chlamydia trachomatis infected women. Mol. Cell. Probes. 11:243-249.

7. Chong, S., D. Jang, X. Song, J. Mahony, A. Petrick, P. Barriga, and M. Chernesky. 2003. Specimen Processing and Concentration of Chlamydia trachomatis Added Can Influence False-Negative Rates in the LCx Assay but Not in the Aptima Combo 2 Assay When Testing for Inhibitors. J. Clin. Microbiol. 41:778-782.

8. Crotchfelt, K. A., B. Pare, C. Gaydos, and T. C. Quinn. 1998. Detection of Chlamydia trachomatis by the Hologic Amplified Chlamydia Trachomatis assay (AMP CT) in urine specimens from men and women and endocervical specimens from women. J. Clin. Microbiol. 36:391-394

9. CUMITECH 31. Verification and Validation of Procedures in the Clinical Microbiology Laboratory.- ASM PRESS, FEBRUARY 1997.

10. Frommell, G. T., R. Rothenberg, S. Wang, and K. McIntosh. 1979. Chlamydial infection of mothers and their infants. Journal of Pediatrics 95:28-32.

11. Gaydos, C.A., T.C. Quinn, D. Willis, A. Weissfeld, E.W. Hook, D.H. Martin, D.V. Ferraro, and J. Schachter. 2003. Performance of the Aptima Combo 2 Assay for Detection of Chlamydia trachomatis and Neisseria gonorrhoeae in Female Urine and Endocervical Swab Specimens. J. Clin. Microbiol. 41: 304-309.

12. Goessens, W. H. F., J. W. Mouton, W. I. Van Der Meijden, S. Deelen, T. H. Van Rijsoort-Vos, N. L. Toom, H. Verbrugh, and R. P. Verkooyen. 1997. Comparison of three commercially-available amplification assays, AMP CT, LCx, and COBAS AMPLICOR, for detection of Chlamydia trachomatis in first-void urine. J. Clin. Microbiol. 35:2628-2633.

13. Holmes, K. K., H. H. Handsfield, S. P. Wang, B. B. Wentworth, M. Turck, J. B. Anderson, and E. R. Alexander. 1975. Etiology of nongonococcal urethritis. NEJM 292:1199-1205.

14. Jaschek, G., C. A. Gaydos, L. E. Welsh, and T. C. Quinn. 1993. Direct detection of Chlamydia trachomatis in urine specimens from symptomatic and asymptomatic men by using a rapid polymerase chain reaction assay. J. Clin. Microbiol. 31:1209-1212.

15. Mahony, J., S. Chong, D. Jang, K. Luinstra, M. Faught, D. Dalby, J. Sellors, and M. Chernesky. 1998. Urine specimens from pregnant and non-pregnant women inhibitory to amplification of Chlamydia trachomatis nucleic acid by PCR, Ligase chain reaction, and transcription-mediated amplification: identification of urinary substances associated with inhibition and removal of inhibitory activity. J. Clin. Microbiol. 36:3122-3126.

16. National Committee for Clinical Laboratory Standards. 1999. NCCLS EP5-A: Evaluation of Precision Performance of Clinical Chemistry Devices; Approved Guideline (Vol. 19, No. 2).

17. National Committee for Clinical Laboratory Standards. 2002. NCCLS EP12-A: User Protocol for Evaluation of Qualitative Test Performance; Approved Guideline for additional guidance on appropriate internal quality control testing practices.

18. Peterson E. M., V. Darrow, J. Blanding, S. Aarnaes, and L. M. de La Maza. 1997. Reproducibility problems with the AMPLICOR PCR Chlamydia trachomatis test, J. Clin. Microbiol. 35:957-959.

19. Schachter, J. 1985. Chlamydiae (Psittacosis-Lymphogranuloma Venereum-Trachoma group), p. 856-862. In E. H. Lennette, et al. (ed.), Manual of Clinical Microbiology, $4^{\text {th }}$ ed. American Society for Microbiology, Washington, D.C.

20. Schachter, J., and M. Grossman. 1981. chlamydial infections. Ann. Rev. Med. 32:45-61.

21. Schachter, J. 1978. Medical progress: chlamydial infections (third of three parts). NEJM 298: 540-549.

22. Schachter, J., E. C. Hill, E. B. King, V. R. Coleman, P. Jones, and K. F. Meyer. 1975. Chlamydial infection in women with cervical dysplasia. Am. J. Obstet. Gynecol. 123:753-757.

23. Stary, A., E. Schuh, M. Kerschbaumer, B. Gotz, and H. Lee. 1998. Performance of transcription-mediated amplification and Ligase chain reaction assays for detection of chlamydial infection in urogenital samples obtained by invasive and noninvasive methods. J. Clin. Microbiol. 36:2666-2670.

24. Toye, B., W. Woods, M. Bobrowska, and K. Ramotar. 1998. Inhibition of PCR in genital and urine specimens submitted for Chlamydia trachomatis testing. J. Clin. Microbiol. 36:2356-2358.

25. Verkooyen, R. P., A. Luijendijk, W. M. Huisman, W. H. F. Goessens, J. A. J. W. Kluytmans, J. H. Rijsoort-Vos, and H. A. Verbrugh. 1996. Detection of PCR inhibitors in cervical specimens by using the AMPLICOR Chlamydia trachomatis assay. J. Clin. Microbiol. 34:3072-3074 
26. Vincelette, J., J. Schirm, M. Bogard, A. Bourgault, D. Luijt, A. Bianchi, P. C. Van Voorst Vader, A. Butcher, and M. Rosenstraus. 1999. Multicenter evaluation of the fully automated COBAS AMPLICOR PCR test for detection of Chlamydia trachomatis in urogenital specimens. J. Clin. Microbiol. 37:74-80.

27. Yuan, Y., Y-X. Zhang, N. G. Watkins, and H. D. Caldwell. 1989. Nucleotide and deduced amino acid sequences for the four variable domains of the major outer membrane proteins of the 15 Chlamydia trachomatis serovars. Infect. Immun. 57:1040-1049.

\section{$M$}

IVD

Hologic, Inc.

10210 Genetic Center Drive

San Diego, CA 92121 USA

U.S. and international contact information:

$\begin{array}{ll}\text { Customer Support: } & +1844 \text { Hologic }(+18444656442) \\ & \begin{array}{l}\text { customersupport@hologic.com } \\ \text { Technical Support: }\end{array} \\ & +18884844747 \\ & \text { molecularsupport@hologic.com }\end{array}$

For more contact information visit www.hologic.com

Hologic, Aptima, Aptima Combo 2, DTS, Leader, PreservCyt, SB100, ThinPrep, Tigris, and TMA are trademarks and/or registered trademarks of Hologic, Inc. and/or its subsidiaries in the United States and/or other countries.

eppendorf (stylized) and REPEATER are trademarks of Eppendorf AG.

KOVA-TROL is a trademark of Hycor Biomedical, Inc.

RAININ is a trademark of Rainin Instrument, LLC.

TECAN and FREEDOM EVO are trademarks of Tecan Group AG

All other trademarks that may appear in this package insert are the property of their respective owners.

This product may be covered by one or more U.S. patents identified at www.hologic.com/patents

(C)2000-2016 Hologic, Inc. All rights reserved.

501799 Rev. 001

2016-03 